\title{
EXCHANGE RATES AND EXPORTS: EVIDENCE FROM MANUFACTURING FIRMS IN THE UK
}

\author{
Xufei Zhang
}

Thesis submitted to the University of Nottingham for the degree of Doctor of Philosophy

April 2008 


\begin{abstract}
The thesis examines the effect of exchange rate variability on firms' export decisions, using data for UK manufacturing firms. We separately investigate the relationship between the changes of level of exchange rate and exports and that between exchange rate uncertainty and exports. Our results show that export extensive margin is not significantly related to changes in the level of exchange rates, whereas exchange rates have a significant and negative impact on the export intensity. Since industry heterogeneity is important in the effect of exchange rate level changes, we further explore possible explanations. In particular, we test whether external orientation and market structure play a role in the effect. Our results provide significant evidence for the role of external orientation and market structure. We also find significant evidence for the hysteresis effect of exchange rate uncertainty on exports, using new measures of uncertainty. In both cases, the behaviour of multinationals is investigated. We find that multinationals are less likely to be negatively affected by both changes of level of exchange rate and exchange rate uncertainty than indigenous firms.
\end{abstract}

It contributes to the micro econometric literature in several aspects. Our evidence for industry heterogeneity from UK firm level data is new. Our explanations for industry heterogeneity by testing two hypotheses are the first attempt to investigate the factors driving different effects across industries. New measures of exchange rate uncertainty and related new method are used to test the hypothesis of hysteresis effects of uncertainty on trade. The use of micro data and new measures enable us to overcome the econometric difficulties and problems in previous studies. We also investigate whether multinationals' export behaviour is different from that of indigenous firms in response to exchange rate fluctuations. As far as we know, the multinationals' ability to deal with currency risk has never been examined before. The thesis provides new evidence for the multinationals' advantage of internalising currency risk over indigenous firms under exchange rate movements. 


\section{Acknowledgements}

I would like to express my gratitude to all those who helped me in completing this thesis. I am deeply indebted to my supervisors Professor David Greenaway and Dr. Richard Kneller for their stimulating suggestions, guidance and encouragement throughout my PhD study. They generously spent their time, energy and insights to help me find this interesting topic and shape my understanding of it. I also wish to thank Sue Berry for her friendly and efficient assistance in organizing those fortnightly supervision meetings over more than three years.

I would like to thank my sponsors, the Leverhulme Centre for Research on Globalisation and Economic Policy, the School of Economics and International Office at the University of Nottingham. Without their financial support, it would not have been possible for me to start my PhD study. Moreover, academic and support staff in GEP and the School provided a friendly and supportive environment for my research during this period.

I thank Professor Marius Brülhart and Dr. Zhihong Yu for their valuable comments. I am also indebted to those who contributed with helpful suggestions during various conferences where parts of this work were presented.

My gratitude also goes to my friends and colleagues at the University of Nottingham, particularly Xiao Qiu, Fangya Xu, Neema Mahabir, and Anuj Mathew for their support and friendship. I am deeply grateful to my dear friend in London, Mahlet Getachew, for her proofreading, friendship and those happy in-depth talks over various topics. Finally, I would like to express very special thanks to my family for their constant support and encouragement. 


\section{CONTENTS}

Abstract

Acknowledgement

$\begin{array}{lll}\text { Contents } & \text { IV }\end{array}$

Chapter 1 Introduction and Overview

1. Introduction, Context and Motivation 1

1.1 Theoretical literature 1

1.2 Empirical background 3

1.3 Firm heterogeneity 5

2. Aim of the Thesis 6

3. Structure of the Thesis 8

Chapter 2 Changes of Exchange Rate Level and Exports

1 Introduction 13

2 Economic Background 15

2.1 Theoretical background 15

$\begin{array}{ll}2.2 \text { Empirical background } & 18\end{array}$

3. Econometric Specification and Estimation Methodology 22

4. Computation of Industry-specific Exchange Rates 28

5. Firm Data and Summary Statistics 37

6. Main Results 45

7. Conclusions 60

Appendix: Definitions of the Variables Used 62

Chapter 3 Explaining Industry Heterogeneity: Controlling for External Orientation

1. Introduction 63

2. Measures of External Orientation and Summary Statistics 66

3. Classification of measures and hypotheses 66

4. Data source, descriptive statistics and correlations 69 
5. Results after Controlling for External Orientation 77

6. Conclusions 83

Appendix 1: Calculating Imported Intermediate Input REER 84

Appendix 2: Calculating Effective External Orientation Index 86

Chapter 4 Explaining Industry Heterogeneity: the Role of Pricing to Market

1. Introduction 89

2. Theoretical Background: Determinants of PTM 90

3. Empirical Literature: Measures of the Determinants 93

4. Measuring the Determinants of PTM 96

4.1 Intra-industry trade index 96

4.2 Computing indictors of intra-industry trade and data sources 98

5. Methodology to Detect Interaction Effects 104

6. Results 110

$\begin{array}{ll}\text { 7. Conclusions } & 119\end{array}$

Chapter 5 Exchange Rate Uncertainty and Exports

1. Introduction 121

2. Theoretical Background 122

2.1 Exchange rate uncertainty and trade 123

2.2 Investment under uncertainty $\quad 124$

3. Empirical Background 126

4. Measuring Exchange Rate Uncertainty 130

5. Empirical Implications and Testing for Hysteresis 136

6. Results 138

7. Conclusions 146

\section{Chapter 6 Conclusions}

1. Summary of Key Findings 147

2. Limitations and Suggestions for Future Research 152

3. Conclusions 156 


\section{Chapter 1}

\section{Introduction and Overview}

\section{Introduction, Context and Motivation}

Firms involved in international business through exporting, importing or FDI are exposed to a set of macro shocks, which is distinct compared to their sales in the domestic market. Exchange rate variations are typically viewed as being an important source of these shocks. Nominal and real exchange rates have fluctuated significantly since the early 1970s following the collapse of the Bretton Woods System of fixed exchange rates. ${ }^{1}$ This volatility has in turn generated increased academic interest in investigating the effects of exchange rate movements on international trade. In particular, empirical studies of the impact of exchange rate uncertainty on trade flows have become an important area within international finance. See IMF (1984) for an early, Cote (1994) and McKenzie (1999) for later surveys, and Clark et al. (2004) for a new and recent literature review on the effects of exchange rate volatility on trade. It is the case, however, that the majority of the empirical evidence is based on macro data. This occurs despite the recent increase in empirical work in international trade that has begun to use firm level micro data to investigate the behaviour of exporters. In this thesis we add to that literature by focusing on the effect of exchange rate movements on firm export behaviour in the UK.

\subsection{Theoretical literature}

\footnotetext{
' See, for example, Bayoumia and Eichengreen (1998, Section 2 and Table 1) for the detailed evidence of the great exchange rate volatility after the Bretton Woods compared to that before the collapse.
} 
There is a small theoretical literature investigating the effects of exchange rate changes on a firm's export decisions and the volume of international trade. A key assumption in these models is that firms must incur a sunk cost when it enters international markets (Campa, 2004). It is first shown by Baldwin (1988) that, when market-entry costs are sunk, large enough temporary exchange rate fluctuations can have hysteresis effects on trade prices and quantities. In the presence of sunk entry costs, there exists a gap between a firm's entry and exit conditions: a firm's entry condition is that its expected profits from exporting exceed the sunk entry cost, it will exit when its expected profits is sufficiently negative. That is crucial to these hysteresis effects. A large enough exchange rate fluctuation can induce entry or exit, which leads to a change in market structure and thus generates persistent effects. Baldwin and Krugman (1989) further formalize and extend these ideas.

Dixit (1989a), Dixit (1989b) and Krugman (1991) model investment decisions under uncertainty in an "option" approach. One of the models' applications is foreign trade under exchange rate uncertainty. By introducing the standard financial economics technique of option appraisal, an exporting firm is regarded as owning an option to leave the export market, and a non-exporter an option to enter. The cost of exercising the option, as well as sunk and variable costs, is considered when a firm decides to enter or exit. Since the value of the option increases with uncertainty, the gap between the trigger point for entry and that for exit will increase with the degree of uncertainty. These gaps produce hysteresis and the size of hysteresis increases with exchange rate volatility. 


\subsection{Empirical background}

Despite the clear placement of firms at the heart of these models of exchange rates and international trade, the empirical evidence is based largely on macro data. In the past thirty years, there have been a large number of studies focusing on finding empirical evidence at an aggregate level of a relationship between exchange rate uncertainty and aggregate trade. Although theoretical models show that there are hysteresis effects of exchange rate uncertainty on trade price and quantities, and the size of gap between exchange rates that trigger entry and exit is increasing with uncertainty, existing empirical studies using macro data just focus on investigating the simple correlation between trade and measures of exchange rate uncertainty. Though many researchers and policy makers believe that exchange rate uncertainty (volatility) may depress trade, early empirical work (see IMF (1984) and McKenzie (1999) for surveys) did not yield consistent results: there exists little or no significant evidence for a negative effect between exchange rate volatility and trade volume. More recent empirical work, for example, Frankel and Wei (1993), Wei (1999), Dell' Ariccia (1999), Rose (2000), and Tenryro (2003), adopting a gravity approach has found some evidence of a negative relationship but reported it as small in magnitude (See Clark et al (2004) for a survey). More detailed empirical work using macro data will be reviewed in Chapter 5.

Of the difficulties of estimating the relationship between exchange rate uncertainty and trade using macro data, three issues are worth highlighting. First, the test for a simple correlation between the two may be misspecified, if, as the theoretical models show, there is a hysteresis effect between exchange rate uncertainty and trade. Second, some of the measures of exchange rate uncertainty used may cause 
potential biases. A typical approach, for example, has been to use conditional variances from a GARCH model or the standard deviation of first differences of the logarithmic spot exchange rate. Such measures do not take into account the expected component of exchange rates and firms' hedge behaviour. The concept of uncertainty should refer to the unexpected portion of a variable only. As measures of uncertainty are crucial in empirically evaluating the relationship, this problem should not be neglected. Finally, recent empirical studies (such as Das, Roberts and Tybout, 2007), as well as recent theoretical work on the uncertainty and investment relationship (such as Darby et al., 1999), show that there is industry heterogeneity in the effects of exchange rate uncertainty on trade (or investment). If there is heterogeneity in the effects, evidence from aggregate data or pooled data may be problematic due to aggregation effects, and thus may provide misleading policy implications. These three issues form the areas of research explored in this thesis.

In using micro data, we build on a small literature using firm data for other countries. Since 1990 s, there has been a rapidly growing literature using firm level data to investigate the relationship between firm's characteristics and entry to and survival in export markets. ${ }^{2}$ Among them, a few papers (such as Campa 2004 and Bernard and Jensen 2004a) have begun to include exchange rates as a determinant to examine the firms export decisions. ${ }^{3}$ However, their treatment of this issue is typically partial. For example, most papers only include the level of exchange rates in their regressions to examine the role of exchange rates on export decisions. The exception is Campa (2004). It is the only paper to include both the level of

\footnotetext{
${ }^{2}$ See, for example, Bernard and Jensen (1995), Roberts and Tybout (1997), Clerides, Lach and Tybout (1998), Bernard and Jensen (1999), and Greenaway and Kneller (2003). See Greenaway and Kneller (2007) for a review of the empirical work on firm heterogeneity and exporting.

${ }^{3}$ Evidence using firm level micro data for the effects of exchange rates on exporting will be reviewed in Chapter 2.
} 
exchange rates and a measure of exchange rate uncertainty. Evidence from these micro data generally suggests that exchange rate effects are important, although trade adjustments may mainly occur through the adjustment of intensive margin rather than through changes in extensive margin. See, for example, Bernard and Jensen (2004), Bugammelli and Infante (2002) and Campa (2004).

However, there is something the existing literature has ignored. Industry heterogeneity in the effects of exchange rate movements is found in Das, Roberts and Tybout (2007) using a simulation exercise for Columbian industries. As pointed out by Greenaway and Kneller (2007), there are also some counter-intuitive results showing that a devaluation can also lead to substantial exit from export markets as in Blalock and Roy (2005). Clearly the assumption of using pooled data is one that should be further investigated. It also introduces a possible and very interesting research direction to fully understand the source of this heterogeneity. If there is industry heterogeneity in the effects of exchange rate movements on exporting, what are the factors driving heterogeneity in the effects?

\subsection{Firm heterogeneity}

In addition to this industry heterogeneity, an important issue that runs through the thesis more generally is that of differences in the effects of exchange rates between firms. In the current period of globalization, FDI and international trade have grown rapidly and the increasing extent of the involvement of multinational firms in this process has been a strong feature. Yet, the exporting responsiveness to exchange rate fluctuation by multinational firms has been unexplored. For example, will exchange rate movements have the same impact on multinational firms' 
exports as on domestic firms' exports for a certain country? There are good reasons to think that it will differ. According to the standard textbooks of international business such as Hill (2005) and Rugman and Collinson (2006), multinational firms can internalise currency risk in many ways: internal co-ordination of their own global activities by internalising their own foreign exchange transactions; internal borrowing and lending internationally among subsidiaries; varying the speed of payments to take advantage of expected currency movements, and so on. So multinationals may be less affected by exchange rate movements. Moreover, if multinationals' export behaviour is different from that of indigenous firms, evidence from data pooling multinationals with indigenous firms may also have a problem of aggregation. So the difference between multinationals and indigenous firms may be a possible source of heterogeneity.

New empirical evidence on this issue is important, given that past evidence suggests that the effects of exchange rate movements on exports are not unambiguously clear, and there are some new and interesting aspects that haven't been studied before. A deeper and closer investigation on the heterogeneity in the effects of exchange rate fluctuation on exports across industries and firms with different ownerships provides the motivation behind this thesis. Moreover, clearer evidence would also help to evaluate the relative merits of fixed or managed exchange rates policy.

\section{Aims of the Thesis}

This thesis uses firm-level data for a sample of UK manufacturing firms to investigate empirically the effects of exchange rate movements on firms' export 
behaviour. Unlike previous studies where the focus is typically one aspect of exchange rate movements, this thesis breaks down effects of exchange rate movements on exports into two different types: effects of changes of exchange rate level and effects of exchange rate uncertainty on export decisions. We discover which aspects of exchange rate movements, i.e. level of exchange rates or/and exchange rate uncertainty, are important to investigate cross industry/firm heterogeneity. We find industry heterogeneity in both relationships. We further our study in trying to explain industry heterogeneity in the effects of exchange rate level changes on firms' export decisions. Two factors, the degree of external orientation (the cost impact of exchange rate) and the market structure (the pricingto-market behaviour of firms), are examined to explain industry heterogeneity. In the chapter on exchange rate uncertainty and export decisions, we use a new method of testing the hypothesis of hysteresis effects by employing novel measures of exchange rate uncertainty. Our method circumvents the three problems of using macro data mentioned previously and attempts to overcome econometric difficulties in testing the hypothesis. In addition, our data enable us to examine a further issue: the export behaviour of multinational firms under exchange rate variability. Our results suggest that multinationals are less likely to be negatively affected by exchange rate movements than indigenous firms. We employ microeconometric methods to deal with firm level data. A two-stage sample selection model, which separately estimates the exchange rate effects on firms' decisions on export markets entry and their decision on export shares after entry, is employed to examine these relationships. 
The thesis aims to contribute to the empirical literature in main aspects below. First, using data for UK manufacturing firms, we find that industry heterogeneity is important in the effects of exchange rate level changes on firms export decisions. This is the first analysis of exchange rate level movements and exports for a most comprehensive panel of UK firms. Only Das, Roberts and Tybout (2007) provide simulation evidence for industry heterogeneity. Our direct results for industry heterogeneity from UK firm level data are new. Second, we try to explain industry heterogeneity by testing two hypotheses: the cost impacts of exchange rate movements and the role of market structure. This, to our knowledge, is the first attempt to investigate the factors influencing the differences in the effects across industries. Obviously the two hypotheses are also new in understanding the relationship between exchange rate and exporting. Third, we use different measures of exchange rate uncertainty and a new method to test the hypothesis of the hysteresis effects of uncertainty on trade. The use of micro data and new measures enable us to overcome the econometric difficulties and problems in previous studies to test the hypothesis. Finally, we investigate whether multinationals' export behaviour is different from that of indigenous firms in response to exchange rate fluctuations. As far as we know, the multinationals' ability to deal with currency risk has never been examined before. This provides new direct evidence for the multinationals' advantage of internalising currency risk over indigenous firms under exchange rate movements.

\section{Structure of the Thesis}

Chapter 2 is the empirical study on changes of exchange rate level and exports relationship. It starts by presenting an initial theoretical framework and 
comprehensive empirical background. Then the econometric methodology employed is introduced and discussed. We apply a sample selection model to separately examine the effects of changes of exchange rate level on export entry for non-exporters (extensive margin) and those on export share for existing exporters (intensive margin). As the exchange rate is the key issue we focus on, we also explain our method of calculating the real effective exchange rate at 3-digit industry level for UK manufacturing industries, which is used as the exchange rate in our later analysis. Then the results are reported, in which we investigate whether ownership (as between foreign and domestic) matters, which among other things offers a new way of examining the export behaviour of multinationals in response to exchange rate variability. Finally, we also find that industry heterogeneity is important in the effects. Effects of exchange rate level changes on exports are different across industries.

Since we find that industry heterogeneity in the effects of exchange rate variability on exports is important in Chapter 2, we then try to explain industry heterogeneity. We explain industry heterogeneity by testing two hypotheses in the next two chapters: one is to control for external orientation, the other is to examine the role of market structure. Our analysis shed new light on explaining industry heterogeneity in effects of exchange rate changes on exports. Our results also provide implications for policy makers: different policies might be employed for different industries with different characteristics.

Chapter 3 controls for cost impact of exchange rate changes. Real exchange rate appreciation obviously makes the export price more expensive, but it also makes 
imported intermediate inputs cheaper, which may lead to cost reduction. Since international trade in intermediate inputs is becoming ever more important, we cannot ignore the effects of exchange rate movement on firms' production costs. So the degree of exposure to external shocks for each industry may play a role. For external orientation (the level of imported intermediate inputs), we rely on two families of indicators for each 2-digit manufacturing industry, intended to proxy for inward orientation and net external orientation. After the introduction of the two families of measures of external orientation, we present our results. We find some evidence for a role of external orientation: industries with a low degree of external orientation are less likely to be negatively affected by exchange rate movements.

Chapter 4 examines the role of market structure, specifically the role of pricing to market (PTM) behaviour. Firms in different markets with different market structures may have different export behaviour in response to exchange rate risk. Market structure is a potential factor to influence firm behaviour. More specifically, under imperfect competition when firms with market power, price setters prefer to keep their prices closely in line with those of local industry competitors. Thus export prices of firms are detached from domestic prices with segmented markets. Firms' PTM behaviour ensures price stability in terms of export destination currency. We borrow the idea of PTM (such as Krugman 1987, Dornbusch 1987, and Marston, 1990) and expect that exports in sectors with a higher degree of PTM are less negatively affected by exchange rate movements in terms of local currencies. The chapter starts by summarizing the theoretical models of PTM, and then looks at measures of PTM used. We choose intra-industry trade type indices as our measures of the determinant of PTM to test whether PTM affects the role of 
exchange rate changes on export decisions. The econometric methods to detect interaction effects between PTM and exchange rate movements on exports are discussed. Our results support the role of PTM: PTM plays some significant role in the relationship between exchange rate movements and exports; the exports in industries with a high level of PTM are less likely to be affected by exchange rate changes.

Chapter 5 is the empirical analysis of the effects of exchange rate uncertainty on exports. It starts with a literature review. Then the empirical methodology and the methods to measure exchange rate uncertainty are presented. It aims to contribute in two respects. One is to test the hypothesis of hysteresis effects of exchange rate uncertainty on firm export behaviour using micro data for UK firms. Theoretical models show that there are hysteresis effects on trade price and quantities. And the size of gap between the exchange rates that trigger entry and exit increases with uncertainty. We try to test the hypothesis of hysteresis. There are difficulties with the empirical test: it is difficult to compute the thresholds of entry and exit decision and examine the relationship between the thresholds and uncertainty; existing papers investigating simple correlations between trade and exchange rate uncertainty may have problems of misspecification; and direct observation on entry and exit are difficult to capture. Here we use new measures of exchange rate uncertainty to overcome the difficulties mentioned above. Our empirical results provide evidence that increased exchange rate uncertainty increases the inertia in firms' export intensity decision, whereas there is no evidence for hysteresis effects in export entry decision. The other contribution is to examine the impacts of 
exchange rate uncertainty on multinational firms. We find that multinationals are less likely to be influenced by exchange rate uncertainty than indigenous firms.

Finally in Chapter 6, we present the main conclusions of the thesis. We summarize our key finding, then discuss the limitations of our empirical analysis on this issue and propose some suggestions for future research. 


\section{Chapter 2}

\section{Changes of Exchange Rate Level and Exports}

\section{Introduction}

As explained in Chapter 1 , the effects of exchange rate movements on exports can be broken down into two kinds of effects: the effects of changes in the level of the exchange rate and the effects of exchange rate uncertainty on exports. This chapter aims to examine the former using data for UK manufacturing firms. Although there is no general equilibrium theoretical model embedded in firm behaviour on this relationship, people generally believe that there is a negative relationship between the level of exchange rates and exports. This is partly fashioned by aggregate models and partly intuitive, as exchange rate appreciation may lead to prices rising in foreign markets and thus may induce decreases in demand. All existing theoretical models in this area focus on the relationship between exchange rate uncertainty and trade, which was briefly introduced in Chapter 1 and will be fully reviewed in Chapter 5. So our literature review will focus mainly on empirical papers on the relationship between exchange rates and trade (and/or exports).

Then we empirically analyze the effects of movements in the exchange rate level on firm decisions on export entry, exit, and export intensity. The analysis breaks down export adjustments between changes in intensive margin and changes in extensive margin. Exchange rates used in this chapter, as well as in the rest of the thesis, are 3-digit industry specific real effective exchange rate (REER) indices from 1988 to 2004 . The dataset for UK manufacturing firms merges the Financial Analysis Made Easy (FAME) database with data from OneSource. The resulting 
dataset is the most comprehensive manufacturing firm level dataset among recent studies on the export behavior of UK manufacturing firms. The firm level dataset also enables us to investigate the effects of changes of exchange rate level on the export behaviour of multinationals. So we also look at whether there are any differences in the effects on the export behaviour between indigenous and multinational firms. In the end, we look at whether the effects of exchange rate changes on exports are different across industries.

This chapter adds to the existing literature in several aspects. First, it offers the first analysis of exchange rate level movements and exports for a large panel of UK firms. Since the UK is the fifth largest exporter of merchandise globally, it is clearly a nontrivial case to investigate. Second, it applies a sample selection model which separately estimates the exchange rate effects on firms' decisions on export markets entry and their decision on export shares after entry (the extensive and intensive margins of trade). Third, we investigate whether ownership (as between foreign and domestic) matters, which among other things offers a new way of examining the export behavior of multinationals in response to exchange rate variability. According to the standard textbooks of international business such as Hill (2005) and Rugman and Collinson (2006), MNEs can internalize currency risk in many ways, and may be less affected by exchange rate movements. However, to our knowledge, there is no evidence on this issue so far. This chapter provides the first evidence on this aspect of multinationals. Fourth, we investigate whether the exchange rate effects are different across industries, which is a new direction for the research on the relationship between exchange rate and exports. If industry 
heterogeneity is important, evidence using macro data or pooled data may be misleading.

Using data for UK manufacturing firms, we find that movements of exchange rate levels have little effect on firm's export participation and exit decisions. However, they do have a significant and negative impact on export intensity after entry. The responsiveness of the export share to exchange rate changes is not quantitatively small: one index point depreciation in the REER index will increases export share by about 1.28 percent. For multinationals, we find that their export behavior is less likely to be affected by exchange rate changes than that of indigenous firms. This difference between domestic and multinational firms offers one possible explanation for the mixed evidence of an effect from exchange rates at the macro level. We also find significant evidence for the industry heterogeneity in the exchange rate effects.

The remainder of the chapter is organized as follows. The next section presents the theoretical and empirical background. Section 3 deals with some estimation and econometric issues. Section 4 introduces our method for computing industry specific REERs. Section 5 presents the firm level data and sample used to estimate the model. Section 6 reports our empirical findings. Finally, Section 7 concludes.

\section{Economic Background}

\subsection{Theoretical background}

To motivate our microeconometric analysis, we first model firm export entry decision. As noted before, sunk costs are vital to this. We characterize sunk costs 
using the dynamic setting introduced by Roberts and Tybout (1997), Bernard and Wagner (2001), Bugamelli and Infante (2003), and Das, Roberts and Tybout (2007). The firm's payoffs from exporting are as follows:

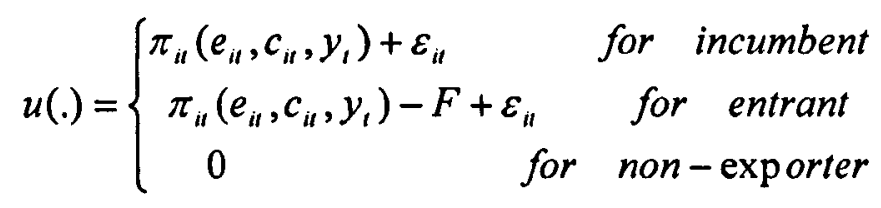

where $F$ denotes sunk costs; $\pi_{i t}$ is profits from export markets, in excess of those from the domestic market, which depends on the exchange rate $\left(e_{i f}\right)$, production costs $\left(c_{i t}\right)$, foreign demand $(y)$, and a serially uncorrelated error term $\left(\varepsilon_{i i}\right)$.

The Bellman equation for the optimal pattern of export market participation is:

$$
\begin{aligned}
\mathrm{V}\left(e_{i t} c_{i t}, y_{t}, \varepsilon_{i t}, E X P_{i(t-1)}\right)= & \underset{E X P_{i} \in\{0,1\}}{\operatorname{EA}}\left\{\pi_{i t}\left(e_{i t,} c_{i t}, y_{t}\right)-\left(1-E X P_{i(t-1)}\right) F\right. \\
& \left.+\delta E_{t} V\left(e_{i(t+l)}, c_{i(t+1)}, y_{t+1}, \varepsilon_{i(t+l)}, E X P_{i t}\right)\right\}
\end{aligned}
$$

where $E_{i i}$ is a dummy variable for firm's export status, which is 1 if firm $i$ exports in year $t$, and 0 otherwise; $\delta$ is the one-period discount rate.

To maximize its expected profit, the firm will export when:

$$
\begin{aligned}
& \pi_{i t}\left(e_{i t}, c_{i t}, y_{t}\right)+\delta\left\{E_{t} V\left(e_{i(t+1)}, c_{i(t+1)}, y_{t+1}, \varepsilon_{i(t+1)}, E X P_{i t} / E X P_{i t}=l\right)\right. \\
& \left.\quad-E_{t} V\left(e_{i(t+1)}, c_{i(t+1)}, y_{t+l}, \varepsilon_{i(t+1)}, E X P_{i t} / E_{i t}=0\right)\right\}+\varepsilon_{i t}>\left(l-E X P_{i(t-l)}\right) F
\end{aligned}
$$

A reduced-form approximation generates the dynamic binary choice of export market participation, where $X_{i t}$ are observable firm characteristics:

$$
\begin{aligned}
E X P_{i t}= & 1 \text { if } \beta X_{i t}+\theta e_{i t}+\lambda E X P_{i(1-l)}+v_{i}+v_{i}+u_{i t}>0 \\
& =0 \text { otherwise }
\end{aligned}
$$


Following Bernard and Wagner (2001), and Das, Roberts and Tybout (2007), sunk costs are taken into account by adding the firm's lagged export status. A positive and significant $\lambda$ implies the presence of sunk costs and persistence of firm export behavior. A significant $\theta$ indicates the effects of exchange rates on firm's export entry decision.

We now turn to the firm's export share decision, conditional on the firm being an exporter. The firm chooses its export share to maximize expected profits from exporting. As an existing exporter, we assume the current export share has no effect on future export share decision. ${ }^{1}$ So the share decision is static and depends on observable firm characteristics and exchange rates. The firm will choose the export share $\rho$ that maximizes

$$
M A X_{\rho} \pi_{i t}\left(\rho_{i t} \mid \rho_{i t}, E X P_{i t}=1\right)
$$

We follow Campa (2004) and model the export share function conditional on being an exporter as the following reduced form:

$$
\rho_{i}=\alpha_{i t} X_{i t}+\alpha_{2} e_{i t}+w_{i t}
$$

$\alpha_{2}$ indicates the effects of exchange rates on firm's export share decision.

There is little systematic theoretical literature exploring the impacts of exchange rate movements on export behavior of multinationals. Clark et al (2004) briefly summarizes papers on an offsetting effect for multinationals, such as Cushman (1983), Clark (1973) and Makin (1978). More systematic explanation of this issue

\footnotetext{
' As pointed out in Campa (2004), due to some real rigidity such as to maintain market share as a favorable objective, there may be the presence of hysteresis on the quantity and export share of export. Froot and Klemperer (1989) provide an example. However, we regard the export share decision as static in this paper.
} 
can be found in standard textbooks of international business, where a multinational enterprise (MNE) can internalize exchange rates fluctuations and minimize the negative effects of exchange rate movements in a number of ways, such as internalising its foreign exchange transactions across countries; internalising investment flows; varying the speed of payments; having more opportunities for hedging currency risk by holding a portfolio of assets and liabilities in different currencies.

To understand further the impact of exchange rate on the export behavior of different types of MNE, we need to investigate further whether there is difference in exchange rate exposure for different types of MNE, as well as differences in methods available to different MNEs. Received wisdom is that there are three types of exchange rate exposure: Transaction Exposure, most which is short term; ${ }^{2}$ Translation Exposure; ${ }^{3}$ and Economic Exposure, which is the extent to which a firm's future international earning power is affected by changes in exchange rates. Translation exposure is the least important of the three. There are a number of strategies available to reduce exposure: hedging in forward markets, leading and lagging payables and receivables, transfer pricing, diversification. Some strategies such as hedging and diversification are available to most MNEs; others may differ for different type of MNEs (horizontal, vertical FDI, export platform and so on).

\subsection{Empirical background}

\footnotetext{
${ }^{2}$ Defined as the extent to which the income from individual transactions is affected by fluctuations in foreign exchange values. Such exposure includes obligations for purchase or sale of goods and services at previously agreed prices and the borrowing or lending of funds in foreign currencies. ${ }^{3}$ It is the impact of currency exchange rate changes on the reported consolidated results and balance sheet of a company. It is basically concerned with the present measurement of past events. It occurs when translating foreign currency financial statement into the reporting currency of the parent company.
} 
We first review evidence from aggregate data examining the relationship between exchange rate volatility and trade. ${ }^{4}$ In general, early work provides little or no evidence of a negative effect of aggregate exchange rate volatility on aggregate trade. For example, Thursby and Thursby (1987) examine the relationship between changes in export volumes and those in exchange rates, and find no significant relationship. Some studies on bilateral trade find some, but not robust, evidence for a negative effect. Recent studies employing gravity models such as Dell' Ariccia (1999) find a negative relationship, but the effects are not dramatic: "complete elimination of volatility would raise trade by a maximum of 15 percent" (Clark et al, 2004). Rose (2000) adopting the same approach also finds a significant negative but small effect using data for 186 countries.

Although macro evidence focuses mainly on exchange rate volatility and trade rather than changes of the exchange rate level and exports, it provides a starting point and throws up some interesting issues, including different effects as between developed and developing countries and differences between multinational and non-multinational companies. As pointed out in Clark et al (2004), for developed countries "where there are well developed forward markets, specific transactions can be easily hedged, reducing exposure" to large exchange rates movements. For multinational firms engaged in a large variety of trade and financial transactions across countries, fluctuations in different exchange rates may have offsetting effects on profitability, and may result in an ameliorated impact of exchange rate movements. As mentioned before, there are some other approaches summarized in international business textbooks for multinational firms to internalize the exchange

\footnotetext{
${ }^{4}$ See Clark et al (2004) for a detailed discussion. A summary table of the studies will be presented in Chapter 5.
} 
rate risks. However, to our knowledge, there is no direct evidence for the effects on export behavior of multinationals. There is an empirical literature on exchange rate variability and FDI decisions. Some studies provide evidence for MNEs abilities to internalize the financing of investments: Lipsey (2001) finds that FDI flows are much more stable during currency crises than other flows of capital; Desai, Foley and Forbes (2004) find that investment, sales and assets of U.S foreign affiliates are significantly more than those of local firms during and after a currency crisis. These papers provide indirect evidence for the internalized or offsetting effects for multinationals.

Studies using micro data have been more successful in finding a relationship between export volumes and exchange rates. Bernard and Jensen (2004a) and Bugamelli and Infante (2003) use the model in Equation (3) to examine the effects of changes of exchange rate level on export market entry. They employ a randomeffects probit model, as well as a linear probability framework. The former requires that the firm specific effects are uncorrelated with the regressors. Potential problems of a linear probability method are well known and fully discussed in Bernard and Jensen (2004a) and Greenaway, Guariglia and Kneller (2007): notably that it fails to properly capture the curvature of the regression function in the proximity of 0 and 1 . This problem may be particularly severe in a dataset with a large number of very high and very low probabilities of exporting. Bernard and Jensen (2004a) find no significant effect of the exchange rate on exports. Bugamelli and Infante (2003) find a small but significant effect: a 1 percent real depreciation raises the probability of exporting by 0.2 percentage points. 
As the only paper focusing solely on this issue, Campa (2004) uses an alternative methodology to estimate the export supply equation with two components: first, export market participation; and second, conditional on being an exporter the relationship between export volume and exchange rate changes. The exchange rate and conditional variance of the exchange rate for firm $i$ are both included. The model estimates export participation as a single equation, which is a dynamic random effects probit model estimated by maximum likelihood. It then estimates the export supply equation after controlling for self-selection into exporting implied by the export participation decision. The lagged export volume of the firm is also included in the export supply estimation to investigate the presence of hysteresis on the quantity of exports. He finds that for Spanish manufacturing firms, coefficients for the level of exchange rate are significant in both estimation processes, whereas exchange rate volatility has insignificant effects. A $10 \%$ depreciation would cause a $7.7 \%$ change in export volume. Most of the change in export volume is due to existing exporters.

Das, Roberts and Tybout (2007) find significant cross-industry variation in the effects of exchange rate movements. Simulating the effect of a 20 per cent devaluation for three Colombian industries they report that the magnitude of the industry response depends on previous export exposure, homogeneity of expected profit flows between firms and their proximity to the export market entry threshold. Ten years after the simulated devaluation, the industry level effect varies between 14 and 107 per cent. Bernard and Jensen (2004b) study the export response of US manufacturing plants to dollar depreciation in the 1980's. They report that 87 per cent of the expansion of exports was from expansion of export intensity amongst 
current exporters and only 13 per cent from entry of new firms. Forbes (2002) studies the impact of a large devaluation on export sales of over 13,500 companies around the world, and finds that on average export sales improve by 4 percent one year after devaluation. Micro evidence shows that changes in exports due to exchange rate level movements come mainly from existing exporters adjusting production.

\section{Econometric Specification and Estimation Methodology}

We examine the effects of changes of exchange rate level on firm export decisions using a sample selection model. As firm characteristics are likely to be correlated with unobserved firm effects, we first estimate a reduced form model within a fixed effects linear probability framework, in which independent variables for firms' main characteristics are included. Following microeconometric papers such as Bernard and Jensen (2004a) and Bugamelli and Infante (2003), we begin by modeling firm's export decisions as:

$$
\begin{aligned}
E X P_{i t}= & a_{0}+a_{1} e m p_{i(t-l)}+a_{2} \text { wage }_{i(t-1)}+a_{3} \text { laborprod } \\
& +a_{6} i n R E E R_{i(t-l)}+a_{4} \text { age } e_{i(t-l)}+u_{i}+e_{i t} E X P_{i(t-l)}
\end{aligned}
$$

where the subscript $i$ denotes firms; and $t$, time. $e m p_{i t}$ represents the logarithm of number of employees as a proxy for firm size. Wage is the ratio of firms' total wage bill to number of employees; laborprod $_{i t}$ represents labor productivity and is the ratio of firm total real sales to number of employees; $E X P_{i t}$ is a dummy representing firm's export status, which equals to 1 if firm $i$ exported in year $t$, and 0 otherwise; $\operatorname{inREER}$ is the 3-digit industry-specific REER. Finally, the error term is made up of two components: $u$, capturing time-invariant firm-specific effects not 
included among the regressors; and $e_{i t}$, an idiosyncratic error term. All timevarying regressors are log lagged one period to reduce possible simultaneity problems. ${ }^{5}$ Industry and time dummies are also included to control for any fixed effects common across industries and years. The definitions of variables are shown in Appendix. As noted earlier, one problem of linear probability estimation is that predicted probabilities may lie outside the $0-1$ range. Moreover, as pointed out in Bernard and Jensen (2004a), fixed effects models produce biased and inconsistent parameter estimates, especially for the coefficient on the lagged dependent variable, but provide a lower bound for the importance of the lagged endogenous variable. Therefore we deploy a random effects probit model:

$$
\begin{aligned}
\operatorname{EXP}_{i t}= & \mathrm{a}_{0}+\mathrm{a}_{1} e m p_{i(t-1)}+\mathrm{a}_{2} \text { wage }_{i(t-1)}+\mathrm{a}_{3} \text { laborprod }_{i(t-1)}+\mathrm{a}_{4} \text { age }_{i(t-l)}+\mathrm{a}_{5} \text { foreign }_{i} \\
& +\mathrm{a}_{6} \operatorname{EXP}_{i(t-l)}+\mathrm{a}_{7} \text { inREER } \\
i(t-1) & +u_{i}+u_{t}+e_{i t}
\end{aligned}
$$

where foreign ${ }_{i}$ is a dummy equal to 1 if the firm is foreign owned, and 0 otherwise; $u_{t}$ is a time-specific component. The use of random effects requires that the firm effects be uncorrelated with the regressors. As some papers have shown, problems may remain, for example, plant characteristics may be correlated with unobserved plant effects, initial period export status may not be exogenous, and there may be sample selection bias. Adding the lagged dependent variable $E X P_{i(t-l)}$ as independent variable may cause endogeneity problem and potential bias. But the problem may be mitigated as our time series is quite long. Here we do not use a

\footnotetext{
${ }^{5}$ For exchange rate index, we also use lagged index. Another reason for using lagged exchange rate index is that, as in the practice of export-import business, the period between placing an order and receiving payment is usually at least as long as three months, the exchange rate observed and used by exporters is usually far earlier than the exact time for firm level export data being listed in datasets. Some people may think expected future exchange rate may be the right exchange rate index. However, we don't think so. Exchange rate is hard to predict and few firms would base their export decisions on probably wrong expected exchange rate predicted by their own. They usually make decisions by observing the current exchange rate and do hedge to minimize their exchange rate risk if necessary.
} 
dynamic pane GMM, as our dependent variable and lagged dependent variable are dummy variables and first-difference instruments may not be appropriate.

Because of sunk costs, exporting can be thought of as a two-stage decision process whereby firms first decide whether to export or not, and second how much to export. The other methodology in a nonstructural framework we employ is a twostage sample selection model, to investigate the effects of some variables on export supply as well as on the decision to export. Our econometric analysis accounts for both decisions and the fact that they are interdependent. It thus avoids any bias resulting from considering them separately. ${ }^{6}$ Two equations are estimated,

$$
\begin{aligned}
& y_{i t}^{*}=x_{i, r-1} \beta+u_{i t} \text { (outcome equation: export intensity/export share); } \\
& d^{*}{ }_{i t}=z_{i, t-1} \gamma+v_{i t} \text { (selection equation: export participation); } \\
& \quad \text { with } \\
& y_{i t}=y_{i t}^{*}{ }_{i t} d_{i t}=1 \\
& y_{i t}=0 \text { if } d_{i t}=0 \\
& \quad \text { and } \\
& d_{i t}=1 \text { if } d^{*}{ }_{i t}>0 \\
& d_{i t}=0 \text { if } d^{*}{ }_{i t} \leq 0
\end{aligned}
$$

Thus, the observed $y_{i t}$, which is the export share, is zero when the firm decides not to export $\left(d_{i t}=0\right)$ and positive when the firm exports $\left(d_{i t}=1\right) \cdot{ }^{7}$ The distribution of

\footnotetext{
${ }^{6}$ Kneller and Pisu (2005) and Karpaty and Kneller (2005) adopt the same methodology.

${ }^{7}$ The dependant variable in Equation 8, export share, is calculated as the ratio of overseas turnover relative to total turnover. It is a firm's percentage of export over total sales, which indicates the degree of export intensity for a firm. Changes in export shares are a combination of changes in a number of elements such as disproportional changes in export quantities relative to total output, changes in market prices and exchange rate changes.
} 
the error terms $\left(u_{i i}, v_{i t}\right)$ is assumed to be bivariate normal with correlation $\rho$. The two equations are related if $\rho \neq 0$. In this case estimating only the export share regression would induce sample selection bias in the estimate of $\beta$ due to the error term $u_{i t}$, and the regressor $x$ would be correlated. To avoid this problem both equations must be estimated via maximum likelihood or a two-step method proposed by Heckman (1979). We employed the former as it is more efficient. ${ }^{8}$ The industry-specific REER, as well as the variables for firm characteristics employed in Equations 6 and 7, is included as independent variable in both equations to examine the effects of level of exchange rate on export participation and export intensity respectively. The only difference for independent variables in both equations (Equations 8 and 9) is the lagged export status dummy. In the export participation equation, we add lagged dependent variable, i.e. the lagged export status (dummy variable), as one of the independent variables to see the role of entry sunk costs, whereas lagged export dummy is excluded in the export intensity equation, as the lagged export status is unimportant when firms have entered export markets and no entry sunk costs are incurred. As pointed out in Campa (2004), due to some real rigidity such as to maintain market share as a favorable objective, there may be the presence of hysteresis on the quantity and export share of export. However, we regard the export share decision as static in this thesis and do not include a lagged dependent variable in the export share equation. The sample selection model is basically static and adding lagged dependent variable requires more advanced econometric technique which is not available for related software at the moment.

\footnotetext{
${ }^{8}$ See Greene (2003) for the discussion.
} 
We should acknowledge some potential limitations of the Heckman selection model. ${ }^{9}$ As the distribution of the error terms from the selection equation and outcome equation are assumed to be bivariate normal, one limitation of estimating the selection model is its sensitivity to the assumption of bivariate normality. A test for the normality assumption in the sample selection model (such as Gourieroux et al. 1987, Chester and Irish 1987, and van der Klaauw and Koning 2003) can be employed. As pointed out in Vella (1998), if normality is rejected, a consistent parameter estimation method may be used such as a semi-nonparametric method proposed by Gallant and Nychka (1987). van der Klaauw and Koning (2003) also suggest a flexible parametric estimation procedure to get consistent estimates robust to non-normality and heteroscedasticity. However, evidence in der Klaauw and Koning (2003) shows that "departures from normality do not cause serous bias in the parameter estimates". So if our interest is the estimates for parameters, especially the exchange rate variable, it is sufficient to assume bivariate normality and straightforwardly obtain estimates. Also as pointed in Vella (1998), semiparametric method is less frequently in practice for two main reasons: one is due to relative difficulty in implementation; the other is that the parametric procedures perform well if the conditional mean of the model is correctly specified.

The most typical concern when using panel data sample selection models has been the presence of unobserved heterogeneity. Heterogeneity across firms and industries may arise for example as a result of different characteristics. These individual characteristics may be unobservable, or may not be measurable. Failure to account for such unobserved heterogeneity may result in biased and inconsistent

\footnotetext{
${ }^{9}$ See Vella (1998) for an excellent survey of the methods for estimating Heckman selection model.
} 
estimates of the parameters of interest. Vella (1998) introduces several methods such as Nijman and Verbeek (1992), Vella and Verbeek (1999) and Kyriazidou (1997) to deal with unobserved heterogeneity. Many papers assume strict exogeneity of the independent variables in two equations. Vella and Verbeek (1999) propose a procedure dealing with binary and censored endogenous variables. It seems attractive to our estimation, as it also allows for the inclusion of the lagged dependent variable in the selection equation. However, existing methods for panel sample selection models are generally in a static framework. Recent papers such as Kyriazidou (2001), Gayle and Viauroux (2007), and Raymond, Mohnen, Palm and van der Loeff (2007) allow for dynamics in both the selection equation and outcome equation. Finally the problem of endogeneity cannot be ignored. Dustmann and Rochina-Barrachina (2000) and Semykina and Wooldridge (2005) suggest procedures to estimate panel sample selection models with continuous endogenous explanatory variables. There are also some general methods taking into account the endogeneity of exporting decisions and other decisions for observable variables such as Maddala (1983) using instrument variables.

Our estimation of the sample selection model with panel data is quite straightforward in a static framework: the maximum likelihood estimation of pooled Heckman selection model with lagged dependant variable, i.e. the lagged export dummy, in the selection equation, together with time and industry dummies in both equations to control for any fixed effects. As discussed before, we do not worry too much about the departure from bivariate normality for the two errors, since our interest is the estimate for the exchange rate parameter. Another reason that we use the straightforward estimates, instead of the semi-nonparametric 
methods for maximum likelihood estimation and the semi-parametric methods for two-step estimation to relax the normality distribution assumption, is that they are computationally cumbersome and require relevant software programs for which the

related econometric work has yet to progress. We are also unable to deal with possible unobserved heterogeneity and full dynamics due to computational demands and absence of appropriate software program. Using lagged independent variables in the sample selection model is the simple way we deal with possible endogeneity.

Within the framework of the sample selection model, the firm's exit from export markets can not be examined. We follow Girma, Greenaway and Kneller (2003), and Alvarez and Görg (2005) to identify the probability of exit using the following discrete model:

$$
\operatorname{Pr}\left(\text { Exit }_{i t}=1\right)=\alpha+\beta \mathrm{X}_{i t}+\theta e_{i t}+\mathrm{d}_{j}+\mathrm{d}_{t}+\varepsilon_{i t}
$$

where Exit it is a dummy variable, equal to 1 if the firm does not export at time $t$ and 0 otherwise. We estimate this model using a Probit. A significant $\theta$ implies the effects of exchange rate movements on the export market exit decision.

\section{Computation of Industry-specific Exchange Rates}

To compute an industry-specific REER, we need to identify: the range of foreign countries to be included as trading partners, their relative weights and the price indices to be used. We use the following equation to compute the industry-specific REER index for each year $t$ :

$$
R E E R_{i}=\prod\left[\left(e_{i t} / e_{t}\right)\left(p_{t} / p_{i t}\right)\right]^{w_{i t}}
$$


Where $e_{i t}$ : Exchange rate of currency $i$ against Special Drawing Rights ${ }^{10}$ (annual average)

(Units of Currency $i$ per SDR in index form, 1995 as the base year)

$\mathrm{e}_{\imath}$ : Exchange rate of GBP against Special Drawing Rights (annual average)

(Units of GBP per SDR in index form, 1995 as the base year)

$\mathrm{p}_{1:}$ Price index of UK (using inflation index as a proxy, 1995 as the base year)

$\mathrm{p}_{i t}$ : Price index of country $i$ (using inflation index as a proxy, 1995 as the base year)

$\mathrm{w}_{i l}$ : the share of exports UK export destination country $i$ within a 3-digit industry

We express the exchange rate in terms of the foreign currency value of a unit of the domestic currency. An upward (downward) movement therefore represents appreciation (depreciation). We compute industry specific REERs in UK for the period from 1988 to 2004 . We choose this period partly because trade data available is OECD bilateral trade commodity data and trade commodity data from $w w w . u k t r a d e i n f o . c o m$ on a consistent classification for that period, and partly because firm level data is also available to us for that period.

Given the time period used this study coincides with a period of large economic and political change, such as the rapid growth of China and India and the opening up of the former Soviet Republic economies, the real exchange rate index that we construct accounts for changes in the exposure of UK exports over time. Choosing time varying export weights in the computation of the REER measure may introduce endogeneity problem, as the weights depend on the movement of

\footnotetext{
${ }^{10}$ The Special Drawing Right (SDR), as defined by IMF, is an international reserve asset, created by the IMF in 1969 to supplement the existing official reserves of member countries. The SDR also serves as the unit of account of the IMF and some other international organizations. Its value is based on a basket of key international currencies. Since the exchange rate data for each currency from IMF is expressed as the value of units per SDR, we use SDR as an intermediary to calculate the exchange rate of each currency against GBP.
} 
exchange rates. To avoid this problem, Campa (2004) uses the average weights throughout the whole period, which implies that the exchange rate index only changes according to the bilateral exchange rate changes between home country and export destinations, whereas trade weight changes across time are ignored. However, we think the endogeneity problem is unlikely to be quantitatively important. As the period we examine is quite long (from 1988 to 2004) and UK's trade partners may change a lot in this long period, using a fixed trade weight for 17 years does not reflect the real degree of exchange rate exposure of UK firms in each sector. So we rely on time varying export weights to compute REER. To check the robustness and the possible endogeneity problem, we also calculate a time invariant average trade weight for each sector throughout the whole period as an alternative. The time invariant REER is highly correlated with our time varying trade weighted REER with a correlation of 0.97 . Results using the alternative REER are similar to those using time varying REER. So we only focus on time varying REER in the thesis hereafter.

Computing export weights: The current classification system of industries in the UK is SIC (2003). As noted already, commodity data is classified according to SITC Rev.3. So we converted SITC commodity data to SIC using the UK SIC (2003) - SITC Rev. 3 concordance after aggregating 5 digit SITC codes to 3 or 4 digit SITC for each 3 digit SIC sector. We then aggregated commodity data to the 3 digit industry level using this concordance, and calculated export weights for each destination for each industry. Following Bernard and Jensen (2004a), the top 25 UK export destinations are chosen as weights. The total percentages of export value for these destinations are always between $80 \%$ and $97 \%$, and therefore 
capture the main drivers of changes in REERs. Moreover, almost all individual trade (export) weights for the 26th export destinations in all industries are less than $1 \%$ during the period.

Data sources for price indices and exchange rates: Nominal exchange rates are annual averages from the IMF, International Financial Statistics. Since the exchange rate data from IFS are exchange rates of currencies in terms of Special Drawing Rights, we use exchange rates per SDR instead of US dollars or other currency. The exchange rates for Taiwan are from the Central Bank of China, Republic of China (Taiwan). The nominal exchange rates are converted to index form with 1995 as the base year.

Several price deflators could be used to calculate REERs: consumer price index (CPI), producer price index (PPI), wholesale price index (WPI), or inflation index and GDP deflator. We use the inflation index for about 170 countries from the IMF, World Economic Outlook Database. The data for the inflation indices are annual averages and the base year is 1995 .

There are 103 three-digit industries. There is no export data for 8 industries and 17 industries with more that 5 percent export value with unknown destination (denoted as 'secret and differences') in some or all of the years. As $5 \%$ is not a small percentage, compared to the percentages of total export value for top 25 export destinations, we cannot ignore the impact of these unknown export destinations on UK firms and make up an artificial weight by ignoring them. Hence, to be as 
precise and accurate as possible, we exclude those industries and end up with REER indices for 78 industries.

Results for REER: Figure 1 shows the REERs for 2-digit industries 31 to 36 as a typical example of movements during the period. Broadly speaking, the indices have moved together and appear to be highly correlated. The distribution of average correlations for each industry is shown in Table 1. Only 6 industries have an average correlation below 0.8 (Industries $172,183,267,283,335$ and 362 ). ${ }^{11}$

Turning to movements of industry specific REERs, troughs appear in 1995 for 72 out of 78 industries, and peaks in 1999 for 63 out of 78 industries. To fully understand REER movements, we need information on export destinations. Table 2 shows each industry's 17 year average of the normalized weights of UK exports to four groups of destinations: the US, Euro area, other European countries, and rest of the world. The average shares of exports to the Euro area and other main European countries are higher than $50 \%$ for almost all industries. The average shares of rest of the world are lower than $25 \%$ for 63 out of 78 industries. Only 5 industries $(160,183,283,335 \text { and } 362)^{12}$ have average shares greater than $40 \%$, most of which are industries with the lowest mean correlation with other industries. Although US shares are not large compared to the Euro area, the US is among the top destinations in many industries. For other countries such as Canada, China, Hong Kong and Singapore, their currencies peg the US dollar during most of the

\footnotetext{
"These industries are: 172 Textile weaving; 183 Dress and dye of fur, and manufacture of fur articles; 267 Cutting,shaping and finishing of stone; 283 Manufacture of steam generators,except boilers; 335 Manufacture of watches and clocks; 362 Manufacture of jewellery and and related articles.

${ }_{12} 160$ Manufacture of tobacco products.
} 
period. So we expect movements of Euro and USD to exert a significant influence on UK REERs.

Figure 1: Industry-specific REER for SIC Industry 31-36

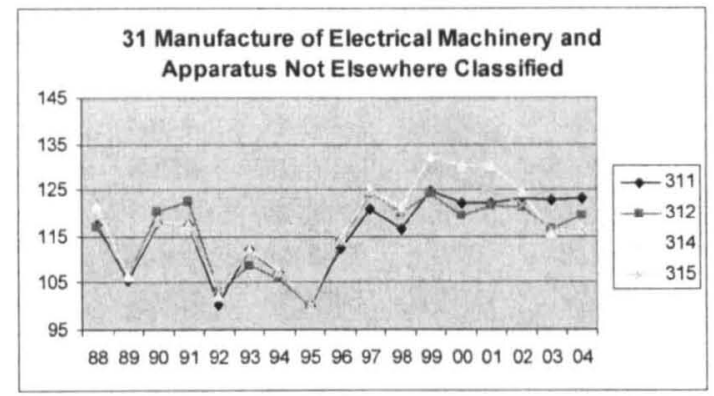

33 Manufacture of Medical, Precision and Optical Instruments, Watches and Clocks
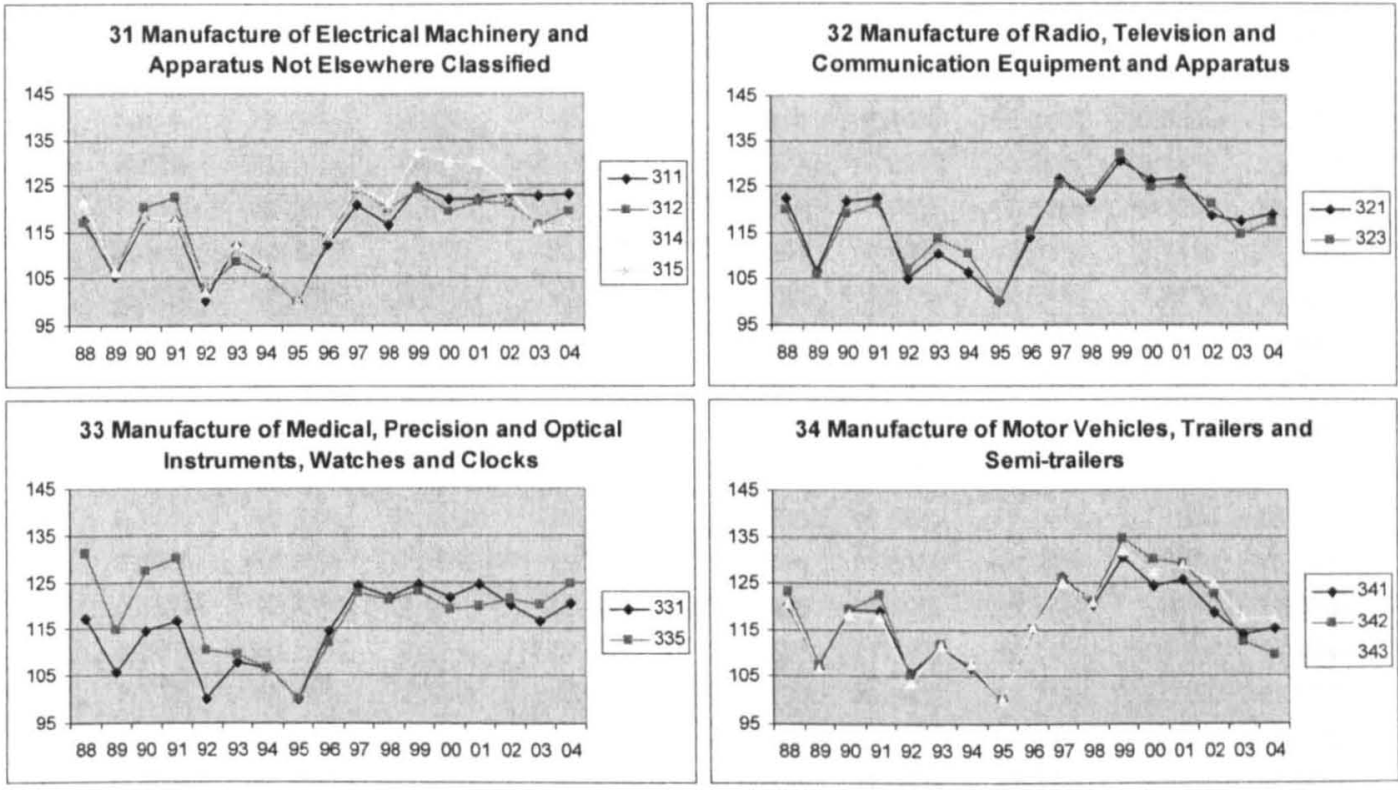

35 Manufacture of Other Transport Equipment

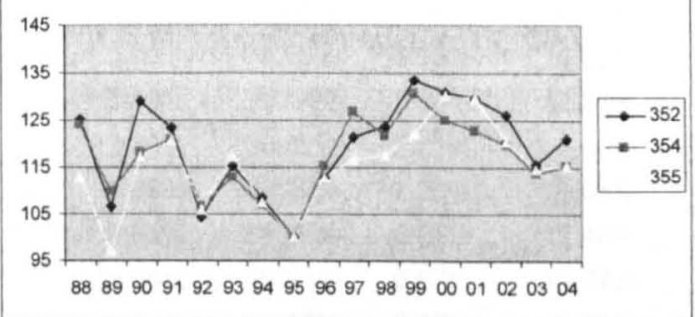

36 Manufacture of Furniture; Manufacturing Not Elsewhere Classified

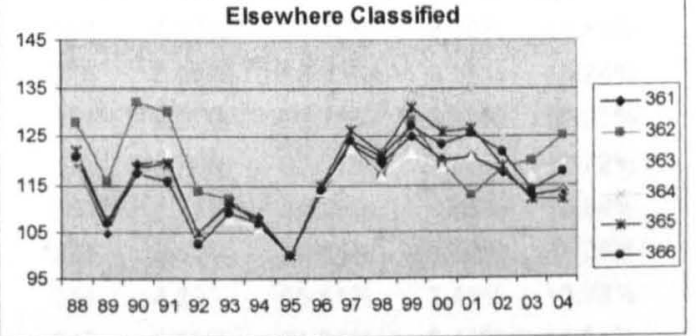

Table 1: Mean of Correlations of REER for Each Industry

\begin{tabular}{cc}
\hline Average Correlation & Number of industries \\
\hline$\geq 0.9$ & 46 \\
$0.8-0.9$ & 26 \\
$<0.8$ & 6 \\
\hline
\end{tabular}

Max average correlation: 0.998 Min average correlation: 0.403 
Table 2: Average Shares of UK Export Destinations for Each Industry (1988-2004)

\begin{tabular}{|c|c|c|c|c|c|c|c|c|c|}
\hline $\begin{array}{c}\text { SIC } \\
\text { code }\end{array}$ & US & $\begin{array}{l}\text { Euro } \\
\text { Zone }\end{array}$ & $\begin{array}{r}\text { Other } \\
\text { EC } \\
\end{array}$ & Rest & $\begin{array}{l}\text { SiC } \\
\text { code }\end{array}$ & US & $\begin{array}{l}\text { Euro } \\
\text { Zone }\end{array}$ & $\begin{array}{r}\text { Other } \\
E C \\
\end{array}$ & Rest \\
\hline 151 & $0.73 \%$ & $82.25 \%$ & $2.40 \%$ & $10.90 \%$ & 265 & $3.05 \%$ & $62.94 \%$ & $9.59 \%$ & $15.75 \%$ \\
\hline 152 & $2.67 \%$ & $77.57 \%$ & $4.88 \%$ & $10.41 \%$ & 266 & $7.73 \%$ & $66.19 \%$ & $4.94 \%$ & $12.97 \%$ \\
\hline 153 & $2.86 \%$ & $71.00 \%$ & $6.65 \%$ & $11.71 \%$ & 267 & $28.05 \%$ & $41.24 \%$ & $3.59 \%$ & $21.14 \%$ \\
\hline 155 & $2.93 \%$ & $69.35 \%$ & $2.13 \%$ & $14.94 \%$ & 268 & $9.65 \%$ & $57.17 \%$ & $6.24 \%$ & $13.79 \%$ \\
\hline 156 & $1.51 \%$ & $75.56 \%$ & $9.33 \%$ & $7.52 \%$ & 271 & $8.44 \%$ & $56.44 \%$ & $10.85 \%$ & $15.20 \%$ \\
\hline 157 & $2.10 \%$ & $72.55 \%$ & $8.84 \%$ & $7.46 \%$ & 273 & $12.62 \%$ & $53.20 \%$ & $9.14 \%$ & $13.37 \%$ \\
\hline 158 & $7.52 \%$ & $53.06 \%$ & $7.31 \%$ & $18.14 \%$ & 274 & $11.00 \%$ & $47.62 \%$ & $9.12 \%$ & $25.04 \%$ \\
\hline 159 & $15.89 \%$ & $40.42 \%$ & $1.83 \%$ & $25.60 \%$ & 281 & $4.78 \%$ & $43.96 \%$ & $8.11 \%$ & $24.23 \%$ \\
\hline *160 & $0.61 \%$ & $47.03 \%$ & $0.29 \%$ & $42.47 \%$ & 282 & $7.27 \%$ & $56.54 \%$ & $7.87 \%$ & $16.58 \%$ \\
\hline 171 & $5.12 \%$ & $63.75 \%$ & $5.45 \%$ & $15.04 \%$ & 283 & $4.27 \%$ & $21.91 \%$ & $5.49 \%$ & $49.99 \%$ \\
\hline 172 & $7.25 \%$ & $42.99 \%$ & $5.43 \%$ & $28.13 \%$ & 287 & $9.78 \%$ & $53.23 \%$ & $10.68 \%$ & $13.71 \%$ \\
\hline 174 & $7.69 \%$ & $63.70 \%$ & $8.88 \%$ & $9.96 \%$ & 291 & $15.82 \%$ & $36.56 \%$ & $8.55 \%$ & $22.82 \%$ \\
\hline 175 & $9.71 \%$ & $52.96 \%$ & $7.82 \%$ & $15.04 \%$ & 292 & $13.24 \%$ & $43.27 \%$ & $6.77 \%$ & $17.61 \%$ \\
\hline 176 & $3.17 \%$ & $60.27 \%$ & $6.26 \%$ & $21.06 \%$ & 293 & $16.88 \%$ & $47.04 \%$ & $7.13 \%$ & $16.82 \%$ \\
\hline 177 & $8.39 \%$ & $67.30 \%$ & $7.36 \%$ & $12.33 \%$ & 294 & $16.31 \%$ & $44.74 \%$ & $6.38 \%$ & $18.33 \%$ \\
\hline 181 & $6.55 \%$ & $72.42 \%$ & $8.56 \%$ & $9.46 \%$ & 295 & $16.40 \%$ & $35.17 \%$ & $6.37 \%$ & $19.38 \%$ \\
\hline 182 & $4.88 \%$ & $60.20 \%$ & $9.22 \%$ & $14.95 \%$ & 286 & $11.34 \%$ & $52.71 \%$ & $6.91 \%$ & $14.28 \%$ \\
\hline *183 & $1.77 \%$ & $42.71 \%$ & $9.31 \%$ & $43.80 \%$ & 297 & $6.04 \%$ & $67.73 \%$ & $5.33 \%$ & $11.99 \%$ \\
\hline 191 & $13.54 \%$ & $40.22 \%$ & $3.56 \%$ & $37.51 \%$ & 300 & $11.51 \%$ & $64.58 \%$ & $8.51 \%$ & $9.15 \%$ \\
\hline 192 & $9.76 \%$ & $55.04 \%$ & $10.10 \%$ & $16.94 \%$ & 311 & $12.98 \%$ & $35.47 \%$ & $5.54 \%$ & $24.60 \%$ \\
\hline 193 & $14.47 \%$ & $57.60 \%$ & $4.55 \%$ & $14.80 \%$ & 312 & $11.90 \%$ & $37.25 \%$ & $6.54 \%$ & $26.86 \%$ \\
\hline 201 & $3.69 \%$ & $78.24 \%$ & $4.98 \%$ & $8.20 \%$ & 314 & $7.50 \%$ & $59.46 \%$ & $9.76 \%$ & $11.41 \%$ \\
\hline 202 & $3.60 \%$ & $74.13 \%$ & $6.18 \%$ & $10.60 \%$ & 315 & $7.90 \%$ & $53.03 \%$ & $9.35 \%$ & $16.24 \%$ \\
\hline 203 & $2.58 \%$ & $75.14 \%$ & $3.32 \%$ & $12.98 \%$ & 321 & $9.97 \%$ & $59.78 \%$ & $5.08 \%$ & $20.61 \%$ \\
\hline 204 & $2.20 \%$ & $83.62 \%$ & $7.21 \%$ & $4.89 \%$ & 323 & $6.76 \%$ & $62.57 \%$ & $7.66 \%$ & $12.72 \%$ \\
\hline 205 & $15.82 \%$ & $52.52 \%$ & $7.90 \%$ & $13.85 \%$ & 331 & $17.31 \%$ & $44.29 \%$ & $6.85 \%$ & $17.84 \%$ \\
\hline 212 & $9.85 \%$ & $64.05 \%$ & $5.28 \%$ & $11.44 \%$ & "335 & $6.62 \%$ & $26.24 \%$ & $16.40 \%$ & $44.35 \%$ \\
\hline 221 & $13.96 \%$ & $40.82 \%$ & $6.67 \%$ & $24.07 \%$ & 341 & $14.23 \%$ & $65.51 \%$ & $3.45 \%$ & $10.28 \%$ \\
\hline 222 & $9.72 \%$ & $51.97 \%$ & $8.67 \%$ & $13.39 \%$ & 342 & $5.29 \%$ & $71.22 \%$ & $5.41 \%$ & $11.61 \%$ \\
\hline 231 & $1.99 \%$ & $29.60 \%$ & $55.12 \%$ & $11.22 \%$ & 343 & $11.01 \%$ & $60.33 \%$ & $5.49 \%$ & $14.10 \%$ \\
\hline 242 & $8.86 \%$ & $47.10 \%$ & $4.80 \%$ & $17.73 \%$ & 352 & $4.50 \%$ & $45.43 \%$ & $11.89 \%$ & $30.66 \%$ \\
\hline 244 & $14.62 \%$ & $48.76 \%$ & $4.71 \%$ & $18.68 \%$ & 354 & $13.79 \%$ & $65.15 \%$ & $7.92 \%$ & $9.48 \%$ \\
\hline 245 & $4.34 \%$ & $55.98 \%$ & $9.41 \%$ & $16.36 \%$ & 355 & $4.67 \%$ & $51.17 \%$ & $8.09 \%$ & $27.72 \%$ \\
\hline 246 & $10.35 \%$ & $49.33 \%$ & $6.81 \%$ & $15.92 \%$ & 361 & $17.92 \%$ & $55.98 \%$ & $7.57 \%$ & $11.22 \%$ \\
\hline 252 & $8.32 \%$ & $58.31 \%$ & $9.24 \%$ & $10.94 \%$ & "362 & $13.98 \%$ & $21.88 \%$ & $15.22 \%$ & $43.30 \%$ \\
\hline 261 & $9.14 \%$ & $58.82 \%$ & $7.90 \%$ & $13.91 \%$ & 363 & $20.42 \%$ & $47.08 \%$ & $6.28 \%$ & $17.41 \%$ \\
\hline 262 & $18.31 \%$ & $37.97 \%$ & $4.20 \%$ & $25.06 \%$ & 364 & $10.31 \%$ & $60.50 \%$ & $13.22 \%$ & $9.56 \%$ \\
\hline 263 & $12.04 \%$ & $50.07 \%$ & $2.01 \%$ & $27.22 \%$ & 365 & $8.79 \%$ & $68.97 \%$ & $7.35 \%$ & $9.03 \%$ \\
\hline 264 & $1.85 \%$ & $67.33 \%$ & $1.73 \%$ & $25.47 \%$ & 366 & $10.92 \%$ & $48.65 \%$ & $7.90 \%$ & $17.78 \%$ \\
\hline
\end{tabular}

Euro area: Austria, France, Germany, Finland, Italy, Belgium, Spain, Greek, Portugal, Netherlands, Ireland, Luxembourg.

Other EC: Denmark, Norway, Switzerland, Sweden

* 5 industries with average share of rest of world greater than $40 \%$. 
Figure 2 show the first differences of logarithms of the REER index for industries 31-36 to investigate changes of REERs for each year. Changes across all industries before 2001 are quite similar, whereas changes after 2001 are quite different. From Figure 3 for log differences of USD and Euro, superficially it is not difficult to find a possible explanation: the changes in the USD and Euro broadly follow the same pattern before 2001, whereas after 2001, the shocks of these two are opposite. So the combination effects of shocks for the two are mixed. The statistics of percentage changes of REER across all industries are shown in Table 3.

Figure 2: First Differences of REER for SIC industry 31-36
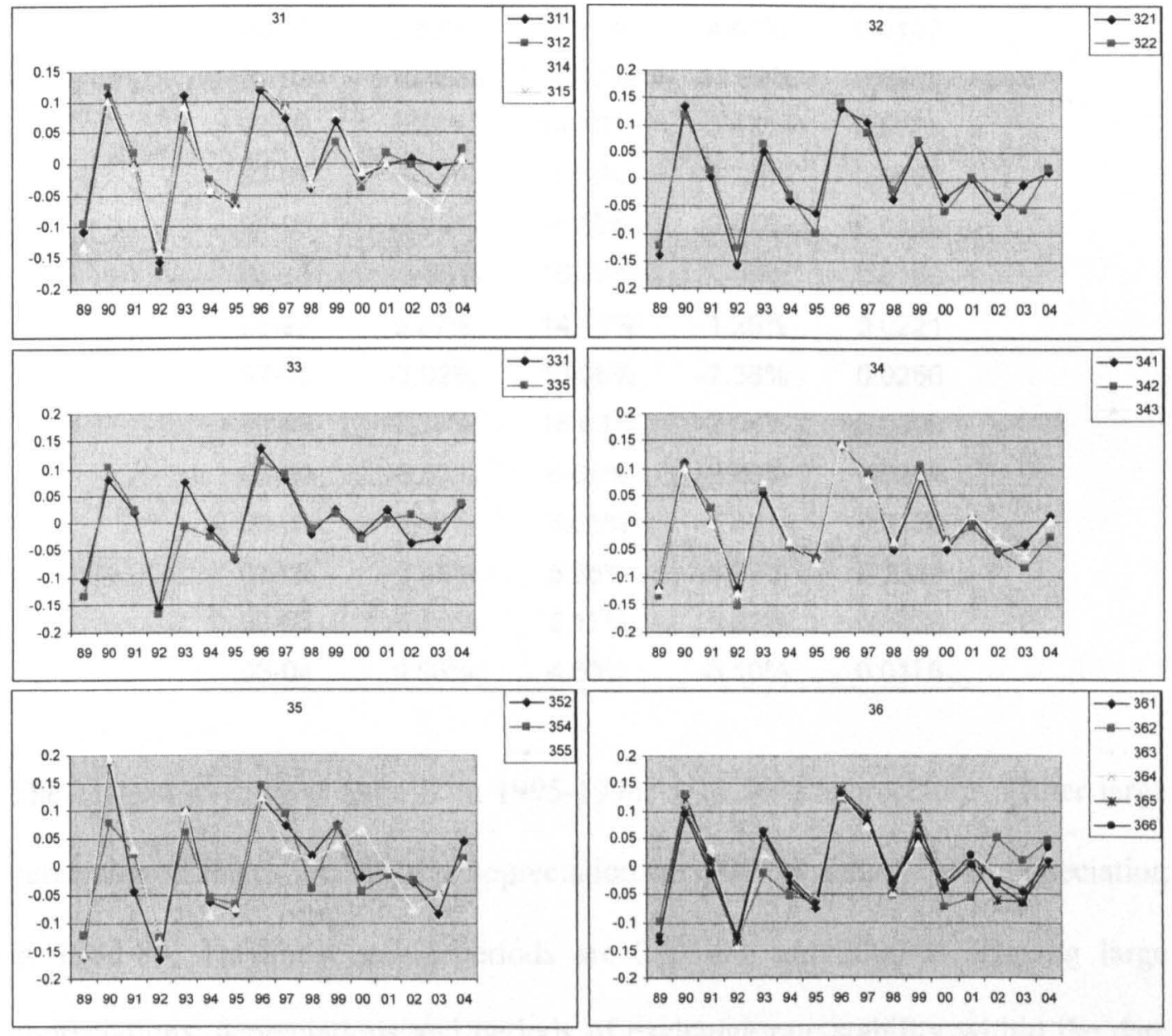
Figure 3: First Differences of Log USD/GBP and EURO(DM)/GBP

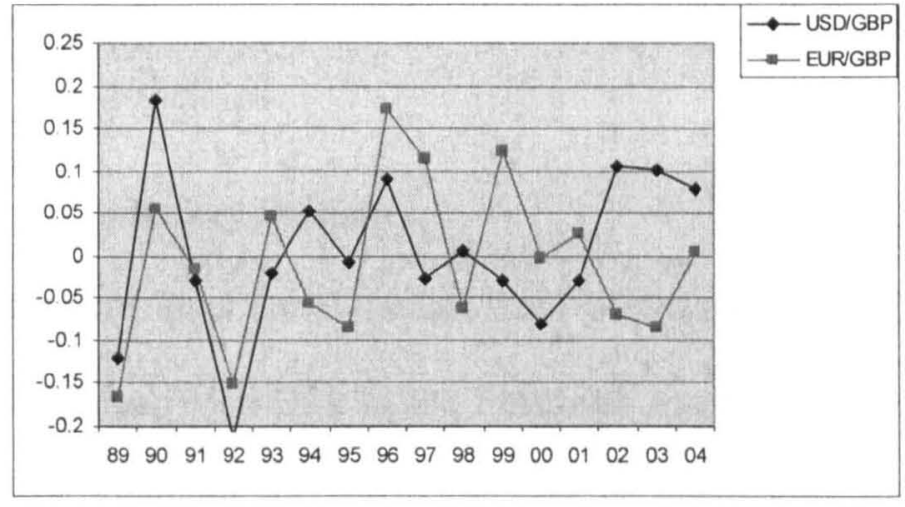

Table 3: Statistics of Percentage Changes of REER across All Industries

\begin{tabular}{ccccc}
\hline Year & Mean & Max & Min & SD \\
\hline $88-89$ & $-11.79 \%$ & $-8.93 \%$ & $-15.07 \%$ & 0.0132 \\
$89-90$ & $12.16 \%$ & $21.20 \%$ & $6.70 \%$ & 0.0276 \\
$90-91$ & $0.89 \%$ & $8.41 \%$ & $-4.41 \%$ & 0.0187 \\
$91-92$ & $-12.59 \%$ & $-6.47 \%$ & $-17.89 \%$ & 0.0206 \\
$92-93$ & $6.27 \%$ & $14.73 \%$ & $-3.48 \%$ & 0.0324 \\
$93-94$ & $-3.55 \%$ & $1.42 \%$ & $-8.25 \%$ & 0.0162 \\
$94-95$ & $-6.48 \%$ & $-4.16 \%$ & $-9.47 \%$ & 0.0100 \\
$95-96$ & $13.56 \%$ & $16.22 \%$ & $7.46 \%$ & 0.0160 \\
$96-97$ & $9.27 \%$ & $14.71 \%$ & $-1.29 \%$ & 0.0221 \\
$97-98$ & $-3.02 \%$ & $13.05 \%$ & $-7.38 \%$ & 0.0256 \\
$98-99$ & $7.38 \%$ & $16.83 \%$ & $-2.04 \%$ & 0.0300 \\
$99-00$ & $-3.52 \%$ & $6.65 \%$ & $-8.89 \%$ & 0.0204 \\
$00-01$ & $0.40 \%$ & $5.65 \%$ & $-5.43 \%$ & 0.0170 \\
$01-02$ & $-3.49 \%$ & $5.85 \%$ & $-8.29 \%$ & 0.0329 \\
$02-03$ & $-5.30 \%$ & $3.17 \%$ & $-9.92 \%$ & 0.0239 \\
$03-04$ & $0.56 \%$ & $4.63 \%$ & $-6.10 \%$ & 0.0176 \\
\hline \hline
\end{tabular}

The biggest average change is in 1995-1996: a 13.56\% appreciation. Other large percentage changes are $12.16 \%$ appreciation in $1989-90$ and $11.79 \%$ depreciation in 1988-89. The most stable periods are 2003-04 and 2000-01. Having large appreciations, depreciations and periods of exchange rate stability within the data makes the period 1988-2004 both interesting and information rich, and provides us 
with an excellent dataset to examine the impact of exchange rate movements on firm export behavior.

\section{Firm Data and Summary Statistics}

We construct our firm level panel dataset from profit and loss and balance sheet data gathered by Bureau Van Dijk in the Financial Analysis Made Easy (FAME) database and from OneSource. Due to the lack of availability of trade data for service industries, we focus only on manufacturing firms. Since firm level data from FAME only covers ten years from 1994 to 2004, we merge the dataset with OneSource which covers 1987 to 2000 . Our panel includes a total of 188,986 annual observations on 23,171 companies. ${ }^{13}$ It has an unbalanced structure, with an average of 8 observations per firm. Table 4 reports the structure of the panel. There are missing values for each key variable. The last figure in each box of Column 1 of Table 7 reports the number of observations for each variable, with the largest number of observations for firm age and smallest for firm intangible assets with about half of the overall observations missing. Table 5 shows the distribution of firm size for the entire sample. Half the observations come from medium-sized firms, micro and small-sized firms take up $27 \%$ and large firms account for $23 \%$. Our dataset has an oversampling of large firms, ${ }^{14}$ which could result in sample selection problems.

\footnotetext{
${ }^{13}$ This is our original number of observations after merging the two datasets. To calculate summary statistics, we use the original one. In order to run our regressions, we have to drop those observations with missing values for each variable in our regressions. So we end up with 44,215 observations in our later regressions.

${ }^{14}$ See Greenaway, Guariglia and Kneller (2007) appendix for the data reporting requirement regulations for partly explanation of the sample selection problem.
} 
Table 4: Structure of the Unbalanced Panel for the Entire Economy

\begin{tabular}{ccrc}
\hline $\begin{array}{c}\text { Number of } \\
\text { Obs. per Firm }\end{array}$ & $\begin{array}{c}\text { Number of } \\
\text { Firms }\end{array}$ & Percent & Cumulative \\
\hline 1 & 1,099 & 4.74 & 4.74 \\
2 & 1,387 & 5.99 & 10.73 \\
3 & 1,334 & 5.76 & 16.49 \\
4 & 1,360 & 5.87 & 22.36 \\
5 & 1,646 & 7.10 & 29.46 \\
6 & 1,595 & 6.88 & 36.34 \\
7 & 1,426 & 6.15 & 42.49 \\
8 & 1,399 & 6.04 & 48.53 \\
9 & 1,702 & 7.35 & 55.88 \\
10 & 5,580 & 24.08 & 79.96 \\
11 & 536 & 2.31 & 82.27 \\
12 & 595 & 2.57 & 84.84 \\
13 & 626 & 2.70 & 87.54 \\
14 & 865 & 3.73 & 91.27 \\
15 & 957 & 4.13 & 95.4 \\
16 & 395 & 1.70 & 97.1 \\
17 & 422 & 1.82 & 98.92 \\
18 & 247 & 1.07 & 100.00 \\
\hline Total & 23,171 & 100.00 & \\
\hline
\end{tabular}

Table 5: Distribution of Firm Size for the Entire Sample

\begin{tabular}{|c|c|c|c|c|}
\hline Size & $\begin{array}{l}\text { Number of } \\
\text { Employee }\end{array}$ & $\begin{array}{l}\text { Number of } \\
\text { Observations }\end{array}$ & Percent & Cum. \\
\hline Micro & $1-9$ & 7,122 & 4.07 & 4.07 \\
\hline Small & $10-49$ & 40,611 & 23.18 & 27.25 \\
\hline Medium & $50-249$ & 86,912 & 49.61 & 76.86 \\
\hline Large & $>=250$ & 40,529 & 23.14 & 100.00 \\
\hline Total & & 175,174 & 100.00 & \\
\hline
\end{tabular}

Table 6 shows the transition of firms in the sample from being an exporter/nonexporter in year 0 to either being an exporter/nonexporter again in year 1 or stopping export/starting exporting. The average percentage of switchers from nonexporter to exporter is about $22 \%$ across the sample, and average percentage of 
switchers from exporter to nonexporter is less than $5 \%$. This shows persistence of firm export behavior.

Table 6: Transition of Firms in the Sample

\begin{tabular}{lccccc}
\hline Year & $\begin{array}{c}\text { Total } \\
\text { firms }\end{array}$ & $\begin{array}{c}\text { Non- } \\
\text { exporter }\end{array}$ & $\begin{array}{c}\text { New } \\
\text { exporter }\end{array}$ & $\begin{array}{c}\text { Always } \\
\text { exports }\end{array}$ & $\begin{array}{c}\text { Firms } \\
\text { exit }\end{array}$ \\
\hline $87-88$ & 1520 & 185 & 162 & 1082 & 91 \\
$88-89$ & 2214 & 313 & 178 & 1609 & 114 \\
$89-90$ & 3494 & 504 & 358 & 2319 & 313 \\
$90-91$ & 4389 & 955 & 295 & 2964 & 175 \\
$91-92$ & 4898 & 1140 & 291 & 3338 & 129 \\
$92-93$ & 5460 & 1212 & 329 & 3747 & 172 \\
$93-94$ & 6701 & 1453 & 538 & 4439 & 271 \\
$94-95$ & 8761 & 1990 & 768 & 5641 & 362 \\
$95-96$ & 9929 & 2532 & 627 & 6490 & 280 \\
$96-97$ & 9841 & 2534 & 565 & 6533 & 209 \\
$97-98$ & 10099 & 2649 & 529 & 6691 & 230 \\
$98-99$ & 9979 & 2738 & 411 & 6607 & 223 \\
$99-00$ & 8573 & 2353 & 417 & 5573 & 230 \\
$00-01$ & 8507 & 2358 & 446 & 5554 & 149 \\
$01-02$ & 8609 & 2426 & 428 & 5588 & 167 \\
$02-03$ & 8407 & 2436 & 367 & 5438 & 166 \\
$03-04$ & 5012 & 1512 & 209 & 3203 & 88 \\
\hline
\end{tabular}

Table 7 reports means, standard deviations, medians and number of observations for the main variables considered. Column 1 refers to the entire sample; Column 2 to firms which never exported; Column 3 to firms that always exported; Column 4 to firms which changed export status. At the mean, exporters are larger than nonexporters, in terms of employees, intangible assets, wages, and sales, and typically older. Export shares are bigger for exporters than switchers. All the medians are lower than the means, indicating positively skewed distributions, highly skewed for sales, size, intangible assets, labor productivity, and export share for switchers (compared with nonexporter and always exporter). Table 8 shows t-tests of differences in means, conditional export premium and t- statistics. Although labor 
productivity is larger for nonexporters in Table 7 , $t$-tests of differences in means

(Table 8) show that the difference between nonexporters and always exporters is

Table 7: Summary Statistics of the Key Variables (mean, overall SD, median and \#obs.)

\begin{tabular}{|c|c|c|c|c|c|c|c|}
\hline & \multirow{2}{*}{$\begin{array}{l}\text { 1. Total } \\
\text { sample }\end{array}$} & \multirow{2}{*}{$\begin{array}{l}\text { 2. Firms } \\
\text { that } \\
\text { never } \\
\text { exported }\end{array}$} & \multirow{2}{*}{$\begin{array}{l}\text { 3. Firms } \\
\text { that } \\
\text { always } \\
\text { exported }\end{array}$} & \multicolumn{4}{|c|}{ 4. Switchers } \\
\hline & & & & $\begin{array}{l}\text { a. Total } \\
\text { sample }\end{array}$ & $\begin{array}{c}\mathrm{b} . \\
\text { Entrants }\end{array}$ & $\begin{array}{l}\text { c. Firms } \\
\text { stop } \\
\text { exporting }\end{array}$ & $\begin{array}{l}\text { d. Firms } \\
\text { keep } \\
\text { switching }\end{array}$ \\
\hline $\begin{array}{l}\text { Real } \\
\text { sales }\end{array}$ & $\begin{array}{l}(7804 \\
7297 . \\
171,8\end{array}$ & $\begin{array}{c}(141024) \\
4341.20 \\
35,744\end{array}$ & $\begin{array}{c}56001.53 \\
(337331) \\
11112.72 \\
64,624\end{array}$ & $\begin{array}{c}62782.94 \\
(392866) \\
7666.72 \\
48,129\end{array}$ & $\begin{array}{c}(406631) \\
7519.95 \\
37,279\end{array}$ & $\begin{array}{c}63334.76 \\
(370857) \\
8934.94 \\
5,223\end{array}$ & $\begin{array}{c}73846.41 \\
(340587) \\
8636.49 \\
4,398\end{array}$ \\
\hline $\begin{array}{l}\text { No. of } \\
\text { emp. }\end{array}$ & $\begin{array}{c}51.934 \\
(3414.92) \\
95 \\
175,174 \\
\end{array}$ & $\begin{array}{c}205.75 \\
(669.013) \\
68 \\
33,494 \\
\end{array}$ & $\begin{array}{c}518.97 \\
(2783.65) \\
132 \\
65,021 \\
\end{array}$ & $\begin{array}{c}500.91 \\
(2697.79) \\
96 \\
49,094 \\
\end{array}$ & $\begin{array}{c}486.88 \\
(2731.15) \\
94 \\
38,421 \\
\end{array}$ & $\begin{array}{c}486.91 \\
(2188.4) \\
112 \\
4,980 \\
\end{array}$ & $\begin{array}{c}654.98 \\
(3080.5) \\
105 \\
4,538\end{array}$ \\
\hline $\begin{array}{c}\text { Real } \\
\text { intang. } \\
\text { assets }\end{array}$ & $\begin{array}{c}7416.11 \\
(117634) \\
0 \\
98,875\end{array}$ & $\begin{array}{c}3043.65 \\
(33840.9) \\
0 \\
18,674\end{array}$ & $\begin{array}{c}7086.97 \\
(101749) \\
0 \\
38,196\end{array}$ & $\begin{array}{c}9440.014 \\
(137548) \\
0 \\
28,340\end{array}$ & $\begin{array}{c}10469.99 \\
(151083) \\
0 \\
22,180\end{array}$ & $\begin{array}{c}6833.85 \\
(81131.8) \\
0 \\
2,783\end{array}$ & $\begin{array}{c}5559.24 \\
(64796) \\
0 \\
2,713\end{array}$ \\
\hline Age & $\begin{array}{c}26.35 \\
(24.33) \\
18 \\
187,376 \\
\end{array}$ & $\begin{array}{c}23.51 \\
(22.71) \\
16 \\
38,040 \\
\end{array}$ & $\begin{array}{c}29.24 \\
(25.93) \\
21 \\
65,185 \\
\end{array}$ & $\begin{array}{c}26.44 \\
(23.815) \\
19 \\
51,558 \\
\end{array}$ & $\begin{array}{c}25.90 \\
(23.48) \\
18 \\
40,208 \\
\end{array}$ & $\begin{array}{c}29.21 \\
(25.97) \\
19 \\
5,293\end{array}$ & $\begin{array}{c}27.80 \\
(23.9) \\
20 \\
4,711\end{array}$ \\
\hline $\begin{array}{l}\text { Labor } \\
\text { prod. }\end{array}$ & $\begin{array}{c}172.22 \\
(2297) \\
78.41 \\
160,101 \\
\end{array}$ & $\begin{array}{c}155.2667 \\
(1264.97) \\
71.98 \\
31,504 \\
\end{array}$ & $\begin{array}{c}147.5264 \\
(1520.13) \\
80.13 \\
63,400 \\
\end{array}$ & $\begin{array}{c}204.0278 \\
(3607.44) \\
80.23 \\
45.814 \\
\end{array}$ & $\begin{array}{c}169.1388 \\
(1346.05) \\
80.14 \\
35,612 \\
\end{array}$ & $\begin{array}{c}180.27 \\
(1024.43) \\
80.63 \\
4,884\end{array}$ & $\begin{array}{c}548.8256 \\
(11133) \\
79.64 \\
4245\end{array}$ \\
\hline $\begin{array}{l}\text { Real } \\
\text { Wage }\end{array}$ & $\begin{array}{c}20.17 \\
(65.33) \\
17.91 \\
174,048\end{array}$ & $\begin{array}{c}19.54068 \\
(16.69) \\
17.24 \\
33,267\end{array}$ & $\begin{array}{c}19.80354 \\
(14.83) \\
18.12 \\
64,815\end{array}$ & $\begin{array}{c}20.34578 \\
(22.29) \\
18.08 \\
48,859\end{array}$ & $\begin{array}{c}20.34565 \\
(23.105) \\
18.12 \\
38.239\end{array}$ & $\begin{array}{c}20.75 \\
(24.62) \\
18.04 \\
4,962\end{array}$ & $\begin{array}{c}19.86549 \\
(11.83) \\
17.75 \\
4,520\end{array}$ \\
\hline $\begin{array}{l}\text { Export } \\
\text { Share }\end{array}$ & $\begin{array}{c}0.221 \\
(0.278) \\
0.184 \\
121,665\end{array}$ & 0 & $\begin{array}{c}0.332 \\
(0.284) \\
0.257 \\
60,069\end{array}$ & $\begin{array}{c}0.1876 \\
(0.264) \\
0.164 \\
36,972\end{array}$ & $\begin{array}{c}0.249 \\
(0.292) \\
0.217 \\
4,160\end{array}$ & $\begin{array}{c}0.132 \\
(0.231) \\
0.013 \\
4,505\end{array}$ & $\begin{array}{c}0.142 \\
(0.2636) \\
0.119 \\
1,163\end{array}$ \\
\hline
\end{tabular}

Note: In each box, mean, overall standard deviation (in the parentheses), median and number of observations are reported from top to bottom respectively.

statistically insignificant. Almost all the t-tests of the differences in means are statistically significant in Table 8. In the last row of Table 8 we follow Bernard and Jensen (1999) in running a regression controlling for other firm level characteristics (employment, wage, age and labor productivity), fixed industry effects and fixed time effects to investigate the conditional export premium and its t- statistic. The 
export premiums are generally positive and significant. The premium for the real wage is significantly negative.

Table 8: T-test of Difference in Means and Conditional Export Premium

\begin{tabular}{lcccccc|}
\hline & $\begin{array}{c}\text { Real } \\
\text { sales }\end{array}$ & $\begin{array}{c}\text { Number } \\
\text { of } \\
\text { employees }\end{array}$ & $\begin{array}{c}\text { Real } \\
\text { intangible } \\
\text { assets }\end{array}$ & Age & $\begin{array}{c}\text { Labor } \\
\text { productivity }\end{array}$ & $\begin{array}{c}\text { Real } \\
\text { Wage }\end{array}$ \\
\hline $\begin{array}{l}\text { Always Export vs. } \\
\text { Never export }\end{array}$ & $23.7^{*}$ & $27.2^{* *}$ & $7.01^{* *}$ & $37.02^{* *}$ & -0.83 & $2.43^{*}$ \\
$\begin{array}{l}\text { Always Export vs. } \\
\text { Switcher }\end{array}$ & $-3.04^{* *}$ & 1.104 & $-2.43^{*}$ & $19.11^{* *}$ & $-3.15^{* *}$ & $-4.66^{* *}$ \\
$\begin{array}{l}\text { Conditional export } \\
\text { premium (t-stat.) }\end{array}$ & $12.52 \%$ & $21.14 \%$ & $10.49 \%$ & $1.16 \%$ & $2.10 \%$ & $-1.206 \%$ \\
\hline
\end{tabular}

Note: 1 . Row 1 and Row 2 show the t-test of difference in means. * indicates significant at 5\%; ** indicates significant at $1 \%$.

2. Row 3 shows the conditional export premium, $t$-statistic is in the parentheses. The regression equation is: $\ln Y_{i t}=a_{0}+a_{1} E X P D U M_{i t}+a_{2} \ln Z_{i t-1}+\sum a_{j} I N D_{j}+\sum a_{i} T_{i}+\varepsilon_{i t}$

Although sales and labor productivity for switchers are the largest among the three categories, the medians are below those of exporters. This is a better measure than the mean for highly skewed distributions. The statistics for the remaining variables for switchers are all between non-exporters and exporters. In Table 7 Subcolumns b, c, and $\mathrm{d}$, we further report statistics for the sub-sample of firms which entered export markets for the first time, firms which stopped exporting for the first time across the period, and firms which switched export status more than twice respectively. These show that except for age, intangible assets and real wage, all are highest for firms which stopped exporting (except for the median of labor productivity). T-tests of differences in means are significantly negative compared to firms that always export. Since these statistics are calculated without separating exporting and nonexporting periods, we further report in Table 9 summary statistics of the variables for switchers, calculating statistics which distinguish exporting 
from nonexporting firm-years within each subgroup of switchers. The table confirms that means for export-year observations are all higher than that for nonexport-year observations except labor productivity and wage for exitors.

Table 9: Summary Statistics of the Key Variables for Switchers

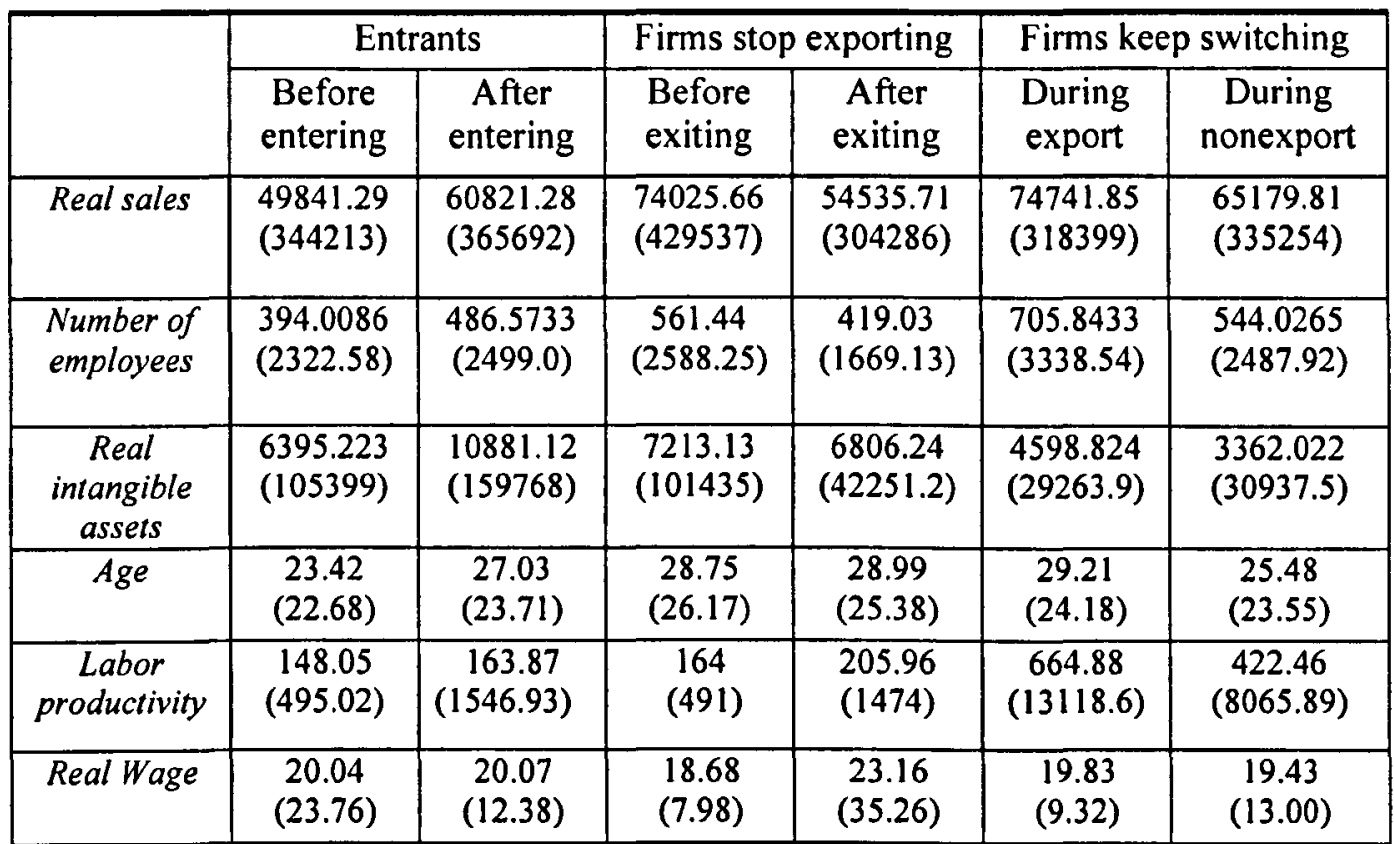

Note: Mean and overall standard deviation (in the parentheses) are shown in each box.

Table 10 compares summary statistics and percentages of exporters by 2-digit industry. The last row in each box shows that the sectors characterized by the highest average percentages of exporting firm-years are medical, precision and optical instruments (83\%), chemicals and chemical products (81\%), and machinery and equipment (81\%). Those characterized by the lowest are wood and products of wood, cork, and plaiting materials (31\%), publishing, printing and reproduction of recorded media (37\%), and food and drink (45\%). The remaining rows report the overall mean of key variables within each industry, the export premium (at the mean) and number of observations. Motor vehicles, trailers and semi-trailers has the highest average annual sales; tobacco products employed the biggest number of employees; fabricated metal products and publishing, printing and reproduction of 
recorded media have the largest number of observations at an average of 20,000 .

There are some negative export premiums and quite large premiums we believe are

due to highly positively skewed distributions.

Table 10: Summary Statistics and Percentages of Exporters by Industry

\begin{tabular}{|c|c|c|c|c|c|c|}
\hline & Real sales & $\begin{array}{l}\text { Number of } \\
\text { employees }\end{array}$ & $\begin{array}{c}\text { Real } \\
\text { intangible } \\
\text { assets }\end{array}$ & Age & $\begin{array}{l}\text { Labor } \\
\text { productivity }\end{array}$ & Real Wage \\
\hline $\begin{array}{l}\text { 15. food } \\
\text { products and } \\
\text { beverages }\end{array}$ & $\begin{array}{r}98837.01 \\
77.548 \% \\
13306 \\
45.06 \%\end{array}$ & $\begin{array}{r}860.5301 \\
74.831 \% \\
13923 \\
45.23 \%\end{array}$ & $\begin{array}{r}16692.03 \\
80.505 \% \\
7298 \\
43.44 \%\end{array}$ & $\begin{array}{r}29.48596 \\
21.115 \% \\
14849 \\
43.47 \%\end{array}$ & $\begin{array}{r}227.9302 \\
44.278 \% \\
12501 \\
46.49 \%\end{array}$ & $\begin{array}{r}17.15078 \\
16.346 \% \\
13841 \\
45.34 \%\end{array}$ \\
\hline $\begin{array}{l}\text { 16. tobacco } \\
\text { products }\end{array}$ & $\begin{array}{r}1012110 \\
-11.648 \% \\
271 \\
71.88 \%\end{array}$ & $\begin{array}{r}6080.582 \\
76.81 \% \\
249 \\
71.70 \%\end{array}$ & $\begin{array}{r}428111 \\
165.87 \% \\
122 \\
74.16 \%\end{array}$ & $\begin{array}{r}44.52055 \\
-20.223 \% \\
292 \\
68.86 \%\end{array}$ & $\begin{array}{r}1592.351 \\
370.61 \% \\
232 \\
75.00 \%\end{array}$ & $\begin{array}{r}24.52172 \\
-26.533 \% \\
244 \\
72.15 \%\end{array}$ \\
\hline 17. textiles & $\begin{array}{r}20116.88 \\
105.69 \% \\
6084 \\
76.88 \%\end{array}$ & $\begin{array}{r}385.4021 \\
98.428 \% \\
6526 \\
76.64 \%\end{array}$ & $\begin{array}{r}424.407 \\
-1.275 \% \\
3345 \\
78.83 \%\end{array}$ & $\begin{array}{r}37.29179 \\
10.925 \% \\
6820 \\
74.89 \%\end{array}$ & $\begin{array}{r}101.6006 \\
-3.672 \% \\
5799 \\
78.48 \%\end{array}$ & $\begin{array}{r}15.4031 \\
4.852 \% \\
6507 \\
76.75 \%\end{array}$ \\
\hline $\begin{array}{l}\text { 18. wearing } \\
\text { apparel; } \\
\text { dressing and } \\
\text { dyeing of fur }\end{array}$ & $\begin{array}{r}25543.5 \\
65.784 \% \\
3711 \\
68.53 \%\end{array}$ & $\begin{array}{r}470.3145 \\
23.609 \% \\
3720 \\
69.27 \%\end{array}$ & $\begin{array}{r}1006.62 \\
174.38 \% \\
1933 \\
68.74 \%\end{array}$ & $\begin{array}{r}26.0266 \\
23.760 \% \\
4060 \\
66.54 \%\end{array}$ & $\begin{array}{r}158.8678 \\
-5.445 \% \\
3354 \\
70.92 \%\end{array}$ & $\begin{array}{r}19.76481 \\
-15.432 \% \\
3691 \\
69.39 \%\end{array}$ \\
\hline $\begin{array}{l}\text { 19. Tanning } \\
\text { and dressing } \\
\text { of leather }\end{array}$ & $\begin{array}{r}25607.73 \\
51.106 \% \\
1291 \\
71.36 \%\end{array}$ & $\begin{array}{r}396.5324 \\
-8.071 \% \\
1313 \\
73.18 \%\end{array}$ & $\begin{array}{r}849.0834 \\
3194.4 \% \\
722 \\
70.14 \%\end{array}$ & $\begin{array}{r}37.34468 \\
12.401 \% \\
1381 \\
70.40 \%\end{array}$ & $\begin{array}{r}265.0157 \\
154.37 \% \\
1213 \\
73.60 \%\end{array}$ & $\begin{array}{r}15.04919 \\
31.661 \% \\
1306 \\
73.12 \%\end{array}$ \\
\hline $\begin{array}{l}20 . \text { wood \& } \\
\text { products of } \\
\text { wood, cork, } \\
\text { and plaiting } \\
\text { materials }\end{array}$ & $\begin{array}{r}16114.08 \\
209.70 \% \\
3385\end{array}$ & $\begin{array}{r}178.4721 \\
169.01 \% \\
3423\end{array}$ & $\begin{array}{r}272.8243 \\
258.83 \% \\
1684\end{array}$ & $\begin{array}{r}27.35183 \\
0.459 \% \\
3874\end{array}$ & $\begin{array}{r}122.9501 \\
14.226 \% \\
2982\end{array}$ & $\begin{array}{r}16.66377 \\
7.916 \% \\
3406 \\
32.47 \%\end{array}$ \\
\hline $\begin{array}{l}\text { 21. pulp } \\
\text { \& paper } \\
\text { products,pub }\end{array}$ & $\begin{array}{r}44673.98 \\
175.72 \% \\
5032\end{array}$ & $\begin{array}{r}334.9411 \\
182.56 \% \\
5350\end{array}$ & $\begin{array}{r}2379.209 \\
566.38 \% \\
2533\end{array}$ & $\begin{array}{r}31.06548 \\
37.119 \% \\
5544\end{array}$ & $\begin{array}{r}136.4919 \\
-32.348 \% \\
4811\end{array}$ & $\begin{array}{r}19.35055 \\
2.983 \% \\
5314\end{array}$ \\
\hline & $56.36 \%$ & $56.22 \%$ & $59.19 \%$ & $54.51 \%$ & $57.56 \%$ & $56.28 \%$ \\
\hline $\begin{array}{l}22 . \\
\text { Publishing, } \\
\text { printing and } \\
\text { reproduction } \\
\text { of recorded } \\
\text { media }\end{array}$ & $\begin{array}{r}25357.69 \\
188.90 \% \\
20730\end{array}$ & $\begin{array}{r}239.9735 \\
123.25 \% \\
19894\end{array}$ & $\begin{array}{r}14913.04 \\
273.26 \% \\
12284\end{array}$ & $\begin{array}{r}25.00072 \\
0.718 \% \\
22149\end{array}$ & $\begin{array}{r}159.9755 \\
55.915 \% \\
18629\end{array}$ & $\begin{array}{r}23.98517 \\
4.521 \% \\
19750\end{array}$ \\
\hline $\begin{array}{l}\text { 23. coke, } \\
\text { refined } \\
\text { petroleum }\end{array}$ & $\begin{array}{r}1602887 \\
19.261 \% \\
730\end{array}$ & $\begin{array}{r}2080.313 \\
75.444 \% \\
718\end{array}$ & $\begin{array}{r}63997.66 \\
-390.73 \% \\
426\end{array}$ & $\begin{array}{r}41.73571 \\
26.270 \% \\
787\end{array}$ & $\begin{array}{r}959.8132 \\
-34.135 \% \\
658\end{array}$ & $\begin{array}{r}26.63382 \\
-4.542 \% \\
709\end{array}$ \\
\hline & $66.49 \%$ & $68.91 \%$ & $70.13 \%$ & $65.21 \%$ & $70.06 \%$ & $69.11 \%$ \\
\hline $\begin{array}{c}24 . \\
\text { chemicals } \\
\text { and }\end{array}$ & $\begin{array}{r}114219.8 \\
39.038 \% \\
13036\end{array}$ & $\begin{array}{r}778.0224 \\
43.619 \% \\
13352\end{array}$ & $\begin{array}{r}13505.27 \\
-4.085 \% \\
7884\end{array}$ & $\begin{array}{r}28.43709 \\
21.954 \% \\
13997\end{array}$ & $\begin{array}{r}272.4934 \\
-22.612 \% \\
12395\end{array}$ & $\begin{array}{r}24.08016 \\
-5.385 \% \\
13255\end{array}$ \\
\hline & $80.54 \%$ & $80.99 \%$ & $81.95 \%$ & $79.06 \%$ & $82.09 \%$ & $81.17 \%$ \\
\hline $\begin{array}{l}\text { 25. rubber } \\
\text { and plastic } \\
\text { products }\end{array}$ & $\begin{array}{r}22837.85 \\
159.83 \% \\
9444 \\
70.22 \%\end{array}$ & $\begin{array}{r}267.9583 \\
119.02 \% \\
9888 \\
70.35 \%\end{array}$ & $\begin{array}{r}1043.916 \\
177.70 \% \\
5727 \\
70.54 \%\end{array}$ & $\begin{array}{r}23.68672 \\
36.877 \% \\
10371 \\
68.49 \%\end{array}$ & $\begin{array}{r}93.68361 \\
6.028 \% \\
8991 \\
71.79 \%\end{array}$ & $\begin{array}{r}17.33123 \\
0.836 \% \\
9852 \\
70.54 \%\end{array}$ \\
\hline
\end{tabular}


Table 10 (cont.)

\begin{tabular}{|c|c|c|c|c|c|c|}
\hline & Real sales & $\begin{array}{l}\text { Number of } \\
\text { employees }\end{array}$ & $\begin{array}{c}\text { Real } \\
\text { intangible } \\
\text { assets }\end{array}$ & Age & $\begin{array}{c}\text { Labor } \\
\text { productivity }\end{array}$ & Real Wage \\
\hline $\begin{array}{l}\text { 26. other non- } \\
\text { metallic } \\
\text { mineral } \\
\text { products }\end{array}$ & $\begin{array}{r}67336.27 \\
81.941 \% \\
4723 \\
57.35 \%\end{array}$ & $\begin{array}{r}680.2011 \\
100.09 \% \\
4952 \\
57.48 \%\end{array}$ & $\begin{array}{r}5672.406 \\
315.34 \% \\
2685 \\
58.17 \%\end{array}$ & $\begin{array}{r}29.45587 \\
7.314 \% \\
5234 \\
55.25 \%\end{array}$ & $\begin{array}{r}98.54662 \\
-15.149 \% \\
4445 \\
58.99 \%\end{array}$ & $\begin{array}{r}18.52681 \\
-2.467 \% \\
4877 \\
57.76 \%\end{array}$ \\
\hline $\begin{array}{c}\text { 27. basic } \\
\text { metals }\end{array}$ & $\begin{array}{r}66150.1 \\
96.154 \% \\
5069 \\
74.54 \%\end{array}$ & $\begin{array}{r}457.4458 \\
77.626 \% \\
5238 \\
74.46 \%\end{array}$ & $\begin{array}{r}1529.135 \\
338.83 \% \\
2964 \\
75.65 \%\end{array}$ & $\begin{array}{r}28.056 \\
10.347 \% \\
5393 \\
73.15 \%\end{array}$ & $\begin{array}{r}459.6961 \\
-26.034 \% \\
4863 \\
75.52 \%\end{array}$ & $\begin{array}{r}24.48454 \\
-5.313 \% \\
5180 \\
74.58 \%\end{array}$ \\
\hline $\begin{array}{r}\text { 28. fabr } \\
\text { metal } p \\
\text { exce } \\
\text { machin }\end{array}$ & $\begin{array}{r}21102.42 \\
215.08 \% \\
21808\end{array}$ & $\begin{array}{r}273.4015 \\
168.43 \% \\
22729\end{array}$ & $\begin{array}{r}1592.126 \\
368.87 \% \\
12713\end{array}$ & $\begin{array}{r}28.07062 \\
37.115 \% \\
24525\end{array}$ & $\begin{array}{r}95.00948 \\
-0.857 \% \\
20119\end{array}$ & $\begin{array}{r}19.18857 \\
-3.182 \% \\
22554\end{array}$ \\
\hline & & $67.45 \%$ & $68.31 \%$ & $64.58 \%$ & $69.17 \%$ & $67.60 \%$ \\
\hline $\begin{array}{l}\text { 29. machinery } \\
\text { \& equipment } \\
\text { not elsewhere } \\
\text { clasified }\end{array}$ & $\begin{array}{r}44926.18 \\
178.01 \% \\
15071 \\
77.89 \%\end{array}$ & $\begin{array}{l}743 \\
6 \% \\
358 \\
0 \%\end{array}$ & $\begin{array}{r}3175.522 \\
51.075 \% \\
8993 \\
79.76 \%\end{array}$ & $\begin{array}{r}26.4155 \\
36.346 \% \\
15935 \\
76.21 \%\end{array}$ & $\begin{array}{r}129.6248 \\
-9.704 \% \\
14335 \\
79.83 \%\end{array}$ & $\begin{array}{r}20.43715 \\
-5.523 \% \\
15273 \\
78.70 \%\end{array}$ \\
\hline $\begin{array}{l}\text { 30. office } \\
\text { machinery and } \\
\text { computers }\end{array}$ & $\begin{array}{r}86319.5 \\
128.52 \% \\
3144 \\
68.82 \%\end{array}$ & $\begin{array}{r}425.1414 \\
81.42 \% \\
3013 \\
71.23 \%\end{array}$ & $\begin{array}{r}3023.364 \\
137.94 \% \\
1696 \\
70.53 \%\end{array}$ & $\begin{array}{r}14.76321 \\
33.523 \% \\
3235 \\
67.78 \%\end{array}$ & $\begin{array}{r}167.3065 \\
-1.757 \% \\
2936 \\
71.75 \%\end{array}$ & $\begin{array}{r}25.09631 \\
-5.324 \% \\
2996 \\
71.50 \%\end{array}$ \\
\hline $\begin{array}{l}31 . \mathrm{e} \\
\text { mach } \\
\text { appar }\end{array}$ & $\begin{array}{r}42325.07 \\
57.904 \% \\
9226\end{array}$ & $\begin{array}{r}511.9408 \\
66.544 \% \\
9327\end{array}$ & $\begin{array}{r}5750.179 \\
132.43 \% \\
5269\end{array}$ & $\begin{array}{r}22.60907 \\
27.722 \% \\
10012\end{array}$ & $\begin{array}{r}121.9972 \\
13.785 \% \\
8594\end{array}$ & $\begin{array}{r}19.62564 \\
-4.141 \% \\
9279\end{array}$ \\
\hline & & & $79.53 \%$ & $74.03 \%$ & $78.48 \%$ & $77.01 \%$ \\
\hline $\begin{array}{r}\text { 32. ra } \\
\text { televi } \\
\text { \&comm }\end{array}$ & $\begin{array}{r}45890.84 \\
116.73 \% \\
5663\end{array}$ & $\begin{array}{r}556.7592 \\
63.781 \% \\
5635\end{array}$ & $\begin{array}{r}10045.72 \\
405.34 \% \\
3377\end{array}$ & $\begin{array}{r}20.04688 \\
36.085 \% \\
5930\end{array}$ & $\begin{array}{r}144.7865 \\
-16.295 \% \\
5340\end{array}$ & $\begin{array}{r}20.41039 \\
-1.345 \% \\
5621\end{array}$ \\
\hline & & & $82.20 \%$ & & $82.41 \%$ & $81.62 \%$ \\
\hline $\begin{array}{l}\text { 33. medical, } \\
\text { precision and } \\
\text { optical } \\
\text { instruments, } \\
\text { watches \& } \\
\text { clocks }\end{array}$ & $\begin{array}{r}28423.08 \\
64.727 \% \\
6934\end{array}$ & $\begin{array}{r}341.7175 \\
69.070 \% \\
7013\end{array}$ & $\begin{array}{r}1517.58 \\
144.49 \% \\
4384\end{array}$ & $\begin{array}{r}22.28633 \\
35.919 \% \\
7383\end{array}$ & $\begin{array}{r}98.0916 \\
-21.105 \% \\
6611\end{array}$ & $\begin{array}{r}21.66466 \\
-8.416 \% \\
6986\end{array}$ \\
\hline $\begin{array}{l}\text { 34. motor } \\
\text { vehicles, } \\
\text { trailers and } \\
\text { semi-trailers }\end{array}$ & $\begin{array}{r}134845.5 \\
1.653 \% \\
4166 \\
71.57 \%\end{array}$ & $\begin{array}{r}906.643 \\
47.070 \% \\
4280 \\
71.02 \%\end{array}$ & $\begin{array}{r}6191.136 \\
76.188 \% \\
2540 \\
70.55 \%\end{array}$ & $\begin{array}{r}22.37166 \\
45.573 \% \\
4383 \\
70.04 \%\end{array}$ & $\begin{array}{r}115.838 \\
-23.999 \% \\
4025 \\
71.96 \%\end{array}$ & $\begin{array}{r}19.07651 \\
0.683 \% \\
4254 \\
71.14 \%\end{array}$ \\
\hline $\begin{array}{l}\text { 35. other } \\
\text { transport } \\
\text { equipment }\end{array}$ & $\begin{array}{r}66921.83 \\
173.86 \% \\
3792 \\
68.86 \%\end{array}$ & $\begin{array}{r}746.0013 \\
127.24 \% \\
3722 \\
69.97 \%\end{array}$ & $\begin{array}{c}21027.1 \\
1913 \% \\
2203 \\
70.88 \%\end{array}$ & $\begin{array}{r}25.8367 \\
70.146 \% \\
3913 \\
67.32 \%\end{array}$ & $\begin{array}{r}694.2221 \\
-26.940 \% \\
3548 \\
71.19 \%\end{array}$ & $\begin{array}{r}20.66197 \\
-8.668 \% \\
3683 \\
70.17 \%\end{array}$ \\
\hline $\begin{array}{l}\text { 36. furniture; } \\
\text { manufacturing } \\
\text { not elsewhere } \\
\text { classified }\end{array}$ & $\begin{array}{r}20939.05 \\
82.968 \% \\
15207 \\
64.22 \%\end{array}$ & $\begin{array}{r}235.6704 \\
59.679 \% \\
15551 \\
65.30 \%\end{array}$ & $\begin{array}{r}2376.74 \\
314.28 \% \\
8093 \\
67.26 \%\end{array}$ & $\begin{array}{r}22.81749 \\
25.840 \% \\
17309 \\
61.82 \%\end{array}$ & $\begin{array}{r}125.4626 \\
-19.541 \% \\
13720 \\
67.34 \%\end{array}$ & $\begin{array}{r}18.76894 \\
-5.967 \% \\
15470 \\
65.38 \%\end{array}$ \\
\hline
\end{tabular}

Note: The overall mean within an industry is listed in the first row, the export premium (measured at mean) is listed in the second row, number of observations in the third row, and the percentage of exporters for firm-year is in the last row in each box. 


\section{Main Results}

Effects of exchange rate movements: Columns 1 and 2 in Table 11 present the results from estimating Equation (4) for the linear probability model and Equation

(5) for the random effects probit. We compare the results with those from a Heckman selection model. For each estimation, results with and without lagged export status dummies are reported in Subcolumns (a) and (b). Of the firm level determinants, a number are consistent with those found in the previous literature. Size and labor productivity always have a significantly positive effect on export participation. The effects of wage and age are insignificant. Foreign owned firms are more likely to export than other firms. The lag of the export dummy in both Column (b)'s has a significant impact on export status next year, which suggests the existence of sunk costs. The coefficient of REERs shows that exchange rate movements did not significantly affect firms' participation behavior, which is consistent with Bernard and Jenson (2004a). Our results are also consistent with those who use subsamples of the same dataset for the UK such as Girma, Greenaway and Kneller (2007), Greenaway and Kneller (2004), and Greenaway, Guariglia and Kneller (2007). Excluding the lagged export dummy allows us to check for robustness of the remaining explanatory variables. The results from this specification are similar to those in column (b) (only the age coefficients become significant), with generally higher levels of statistical significance. Exchange rate movements again have little impact on firm export participation. One point should be noted: the between and within R-squares in the fixed-effects linear probability model in Column 1 are 0.83 and 0.17 respectively. For goodness of fit, the within $\mathrm{R}$-square of 0.17 is directly relevant, which is quite low. The high between Rsquares suggest that correlation between means of our explanatory variables and 
those of export entry of firms are high. However, the limitations of linear probability model and including lagged dependent variable also play a role in $\mathrm{R}$ squares.

Table 11: Models of Export Entry and Exit

\begin{tabular}{|c|c|c|c|c|c|}
\hline & \multicolumn{2}{|c|}{$\begin{array}{l}\text { (1)Entry: Fixed-effects } \\
\text { Linear probability } \\
\text { (t statistic) }\end{array}$} & \multicolumn{2}{|c|}{$\begin{array}{c}\text { (2)Entry: Ramdom-effects } \\
\text { Probit } \\
\text { (z statistic) }\end{array}$} & \multirow{2}{*}{$\begin{array}{c}\text { (3)Exit: } \\
\text { Ramdom- } \\
\text { effects } \\
\text { Probit } \\
\text { (z statistic) }\end{array}$} \\
\hline & (a) & (b) & (a) & (b) & \\
\hline $\begin{array}{l}\text { Lag Industry } \\
\text { REER }\end{array}$ & $\begin{array}{c}0.00048 \\
(0.94)\end{array}$ & $\begin{array}{l}0.00037 \\
(0.80)\end{array}$ & $\begin{array}{l}0.0037 \\
(0.54)\end{array}$ & $\begin{array}{l}0.0026 \\
(0.42)\end{array}$ & $\begin{array}{l}-0.0086 \\
(-1.00)\end{array}$ \\
\hline $\begin{array}{l}\text { Lag log of } \\
\text { wage }\end{array}$ & $\begin{array}{l}-0.0082 \\
(-1.36)\end{array}$ & $\begin{array}{l}-0.0033 \\
(-0.60)\end{array}$ & $\begin{array}{l}-0.062 \\
(-0.95)\end{array}$ & $\begin{array}{l}0.0365 \\
(0.76)\end{array}$ & $\begin{array}{l}-0.045 \\
(-0.70)\end{array}$ \\
\hline $\begin{array}{l}\text { Lag log of } \\
\text { employment }\end{array}$ & $\begin{array}{c}0.045 \\
(17.39)^{* * *}\end{array}$ & $\begin{array}{c}0.0186 \\
(7.74)^{* * *}\end{array}$ & $\begin{array}{c}0.368 \\
(18.80)^{* * *}\end{array}$ & $\begin{array}{c}0.0857 \\
(6.53)^{* * *}\end{array}$ & $\begin{array}{l}-0.0029 \\
(-0.20)\end{array}$ \\
\hline $\begin{array}{l}\text { Lag log of } \\
\text { labor } \\
\text { productivity }\end{array}$ & $\begin{array}{c}0.026 \\
(6.82)^{* * *}\end{array}$ & $\begin{array}{r}0.0075 \\
(2.16)^{* *}\end{array}$ & $\begin{array}{c}0.274 \\
(7.43)^{* * *}\end{array}$ & $\begin{array}{c}0.064 \\
(2.52)^{* *}\end{array}$ & $\begin{array}{l}0.0451 \\
(1.39)\end{array}$ \\
\hline$\underset{\text { age }}{\operatorname{Lag} \log \text { of }}$ & $\begin{array}{c}0.011 \\
(2.71)^{* * *}\end{array}$ & $\begin{array}{c}0.00006 \\
(0.02)\end{array}$ & $\begin{array}{c}0.0953 \\
(3.56)^{* * *}\end{array}$ & $\begin{array}{c}0.00134 \\
(0.09)\end{array}$ & $\begin{array}{l}-0.0003 \\
(-0.02)\end{array}$ \\
\hline $\begin{array}{l}\text { Foreign } \\
\text { owner } \\
\text { dummy }\end{array}$ & & & $\begin{array}{c}0.4001 \\
(5.49)^{* * *}\end{array}$ & $\begin{array}{c}0.2022 \\
(5.43)^{* * *}\end{array}$ & $\begin{array}{c}-0.152 \\
(-3.82)^{* * *}\end{array}$ \\
\hline $\begin{array}{l}\text { Lag Export } \\
\text { dummy }\end{array}$ & & $\begin{array}{c}0.3565 \\
(87.6)^{* * *}\end{array}$ & & $\begin{array}{c}2.888 \\
(93.2)^{* * *}\end{array}$ & \\
\hline Wald chi2 & & & 1885.63 & 11095.08 & 107.18 \\
\hline $\begin{array}{c}\text { Number of } \\
\text { firms }\end{array}$ & \multicolumn{2}{|c|}{5,876} & \multicolumn{2}{|c|}{5,876} & 4,238 \\
\hline $\begin{array}{l}\text { Number of } \\
\text { observations }\end{array}$ & \multicolumn{2}{|c|}{44,215} & \multicolumn{2}{|c|}{44,215} & 33,529 \\
\hline
\end{tabular}

(i) (a) reports results without lagged export status dummy,

(b) reports those with lagged export status dummies.

(ii) * indicates significant at 10\%; ** indicates significant at 5\%; ${ }^{* *}$ indicates significant at $1 \%$ 
Column 3 in Table 11 shows the effects of exchange rates on firm export market exit. We exclude nonexporters and new exporters in our sample. The only significant coefficient is foreign ownership, which is negative and implies a foreign owned firm is less likely to exit from export markets. The coefficient for industry specific REER is insignificant, which shows little impact of exchange rate changes on exit decisions.

However, the rest of the coefficients for firm characteristics are insignificant, which is inconsistent with the related literature. All the industry dummies have highly significant coefficients, suggesting that industry heterogeneity may play an important role on a firm's exit decision as shown in Das, Roberts, and Tybout (2007).

Table 12 reports results for the sample selection model. Column 1 report results from a specification in which we exclude the exchange rate variable. In the first subcolumn, the coefficient on previous export experience is always positive and highly significant suggesting that export participation depends strongly on previous export status. The statistics indicate that the probability of exporting is increasing in the size of the firm. This may reflect the fact that large firms are more likely to be able to compete successfully in international markets. The coefficients of wage and labor productivity are positive as expected, but insignificant. This may be due to controlling for selection bias and is consistent with Kneller and Pisu (2005) using the same methodology for a subsample of the data. 
Table 12: Heckman Selection Model (MLE)

\begin{tabular}{|c|c|c|c|c|c|c|}
\hline & \multicolumn{2}{|c|}{$\begin{array}{l}\text { (1) Heckman } \\
\text { Selection Without } \\
\text { REER }\end{array}$} & \multicolumn{2}{|c|}{$\begin{array}{c}\text { (2) Heckman Selection } \\
\text { with REER }\end{array}$} & \multicolumn{2}{|c|}{$\begin{array}{l}\text { (3) Heckman Selection } \\
\text { (interact with } \\
\text { productivity) }\end{array}$} \\
\hline & $\begin{array}{l}\text { Export } \\
\text { Dummy }\end{array}$ & $\begin{array}{l}\text { Export } \\
\text { Share }\end{array}$ & $\begin{array}{l}\text { Export } \\
\text { Dummy }\end{array}$ & $\begin{array}{l}\text { Export } \\
\text { Share }\end{array}$ & $\begin{array}{l}\text { Export } \\
\text { Dummy }\end{array}$ & $\begin{array}{l}\text { Export } \\
\text { Share }\end{array}$ \\
\hline $\begin{array}{l}\text { Lag Export } \\
\text { dummy }\end{array}$ & $\begin{array}{c}3.04 \\
(39.85) \\
* * *\end{array}$ & & $\begin{array}{c}3.04 \\
(39.94) \\
* * *\end{array}$ & & $\begin{array}{c}3.04 \\
(39.95) \\
* * *\end{array}$ & \\
\hline $\begin{array}{l}\text { Lag Industry } \\
\text { REER }\end{array}$ & & & $\begin{array}{c}0.00214 \\
(0.33)\end{array}$ & $\begin{array}{c}-0.0039 \\
(-2.02) \\
* *\end{array}$ & $\begin{array}{c}- \\
0.00206 \\
(-0.23)\end{array}$ & $\begin{array}{c}-0.0024 \\
(-0.77)\end{array}$ \\
\hline $\begin{array}{l}\text { Lag log of } \\
\text { employment }\end{array}$ & $\begin{array}{c}0.0435 \\
(2.32)^{* *}\end{array}$ & $\begin{array}{l}0.0019 \\
(0.39)\end{array}$ & $\begin{array}{l}0.0435 \\
(2.32)^{* *}\end{array}$ & $\begin{array}{c}0.00207 \\
(0.44)\end{array}$ & $\begin{array}{c}0.0436 \\
(2.32)^{* *}\end{array}$ & $\begin{array}{c}0.0021 \\
(0.43)\end{array}$ \\
\hline $\begin{array}{l}\text { Lag log of } \\
\text { wage }\end{array}$ & $\begin{array}{l}0.0358 \\
(0.73)\end{array}$ & $\begin{array}{c}0.0927 \\
(3.12) \\
* * *\end{array}$ & $\begin{array}{l}0.0362 \\
(0.73)\end{array}$ & $\begin{array}{c}0.0916 \\
(3.09) \\
* * *\end{array}$ & $\begin{array}{l}0.037 \\
(0.75)\end{array}$ & $\begin{array}{c}0.0913 \\
(3.09) \\
* * *\end{array}$ \\
\hline $\begin{array}{l}\text { Lag log of } \\
\text { labor } \\
\text { productivity }\end{array}$ & $\begin{array}{l}0.0375 \\
(1.20)\end{array}$ & $\begin{array}{l}-0.011 \\
(-1.14)\end{array}$ & $\begin{array}{r}0.0375 \\
(1.20)\end{array}$ & $\begin{array}{l}-0.0102 \\
(-1.09)\end{array}$ & $\begin{array}{c}-0.0719 \\
(-0.47)\end{array}$ & $\begin{array}{l}0.0284 \\
(0.48)\end{array}$ \\
\hline $\begin{array}{c}\text { Lag log of } \\
\text { age }\end{array}$ & $\begin{array}{l}-0.025 \\
(-1.57)\end{array}$ & $\begin{array}{c}-0.01 \\
(-2.50) \\
* *\end{array}$ & $\begin{array}{l}-0.0244 \\
(-1.56)\end{array}$ & $\begin{array}{c}-0.0096 \\
(-2.53) \\
* *\end{array}$ & $\begin{array}{c}-0.0246 \\
(-1.57)\end{array}$ & $\begin{array}{c}-0.0096 \\
(-2.52) \\
* *\end{array}$ \\
\hline $\begin{array}{l}\text { Foreign } \\
\text { owner } \\
\text { dummy }\end{array}$ & $\begin{array}{c}0.1317 \\
(4.27) \\
* * *\end{array}$ & $\begin{array}{c}0.058 \\
(6.82) \\
* * * \\
\end{array}$ & $\begin{array}{c}0.1316 \\
(4.26)^{* * *}\end{array}$ & $\begin{array}{c}0.058 \\
(6.80) \\
* * *\end{array}$ & $\begin{array}{c}0.1315 \\
(4.26) \\
* * *\end{array}$ & $\begin{array}{c}0.058 \\
(6.80) \\
* * *\end{array}$ \\
\hline $\begin{array}{l}\text { InREER* } \\
\text { Labor prod. }\end{array}$ & & & & & $\begin{array}{c}0.00093 \\
(0.71)\end{array}$ & $\begin{array}{c}-0.0003 \\
(-0.64)\end{array}$ \\
\hline $\begin{array}{c}\text { Lambda } \\
\text { (standard } \\
\text { error) } \\
\end{array}$ & \multicolumn{2}{|c|}{$\begin{array}{c}-0.034 \\
(0.006)^{* * *}\end{array}$} & \multicolumn{2}{|c|}{$\begin{array}{c}-0.0341 \\
(0.0057)^{* * *}\end{array}$} & \multicolumn{2}{|c|}{$\begin{array}{c}-0.0341 \\
(0.0057)^{* * *}\end{array}$} \\
\hline $\begin{array}{c}\text { Rho } \\
\text { (standard } \\
\text { error) }\end{array}$ & \multicolumn{2}{|c|}{$\begin{array}{c}-0.133 \\
(0.02)^{* * *}\end{array}$} & \multicolumn{2}{|c|}{$\begin{array}{c}-0.1331 \\
(0.0210)^{* * *}\end{array}$} & \multicolumn{2}{|c|}{$\begin{array}{c}-0.1331 \\
(0.0210)^{* * *}\end{array}$} \\
\hline
\end{tabular}

\section{Observations: $44,251 \quad$ Firms: 5, 876}

(i) $\mathrm{Z}$ statistics in parentheses, robust standard errors adjusted for 83 clusters in 3-digit industries.

(ii) *significant at $10 \%$; ${ }^{* *}$ significant at $5 \% ; * * *$ significant at $1 \%$

(iii) $p$ is the estimated correlation between the error terms of the two equations; if it is different from zero it suggests that the two equations are related and that the selection model is appropriate; $\lambda$ is the estimated coefficients of the inverse Mills ratio; if it is different from zero it suggests that there is sample selection.

The second subcolumn reports results for the export share equation. It tells a different story: the effect of size becomes insignificant, the effects of wages 
become significant, and the coefficient of age is negative as before but significant. Foreign ownership has a significant coefficient in both equations as expected. Foreign country dummies are very important both in the participation and export share decision, which is consistent with Kneller and Pisu (2005) and the theory of Baldwin and Ottaviano (2001). Multiproduct firms use trade costs to reduce intervariety competition by placing production of some varieties abroad. Since the varieties are differentiated, all varieties are sold in all markets. Thus FDI/multinationals create trade via reverse imports. Foreign firms in the host country are more likely to be involved in exporting to other countries.

Column 2 reports the effects of including the exchange rate as an independent exogenous variable. Adding this has little impact on other coefficients, which implies that the level of the exchange rate is independent of other variables. The coefficients on the exchange rate are never significant in the export participation equation, which is not consistent with Campa (2004), but is consistent with other empirical evidence referred to in Section 2. However, exchange rate movements have a significant impact on firms' export share decisions with expected signs and significant coefficients in the export share equation even after controlling for industry clustering. ${ }^{15}$ The results suggest that although the exchange rate does not significantly affect a firm's decision on export participation, it does significantly influence the intensity of exports. Or put differently, adjustment is primarily on the intensive margin of trade. Export adjustments to changes of exchange rates are

\footnotetext{
${ }^{15}$ Since our exchange rate is industry-specific REER, industry clustered adjustment may mitigate the effects of exchange rate on export. The sample selection models we use hereafter throughout the thesis are all controlled for industry clustering.
} 
mainly made by existing exporters. This is consistent with the microeconomic findings of Campa (2004) and Bernard and Jensen (2004b).

There may be an effect on the most productive non-exporting firms (i.e. firms whose productivity is just below the cut-off necessary to make positive profits from exporting). To capture this we interact firms' labor productivity with industry specific REERs. The results in Column 3 of Table 12 show the interaction term is insignificant and positive in the export participation regression, which suggest little effect of REER on export entry for marginal firms.

Table 13: Marginal Effects of the Heckman Selection Model (clustered)
\begin{tabular}{|c|c|c|}
\hline & $\begin{array}{c}\text { Export } \\
\text { Dummy }\end{array}$ & Export Share \\
$\qquad \begin{array}{c}\text { Lag Export } \\
\text { dummy }\end{array}$ & $\begin{array}{c}0.817 \\
(0.0115)^{* * *}\end{array}$ & \\
$\qquad \begin{array}{c}\text { Lag Industry } \\
\text { REER }\end{array}$ & 0.00038 & -0.0034 \\
$(0.00115)$ & $(0.002)^{*}$ \\
\hline Lag log of & 0.0078 & 0.00456 \\
employment & $(0.0035)^{* *}$ & $(0.00455)$ \\
\hline $\begin{array}{c}\text { Lag log of } \\
\text { wage }\end{array}$ & 0.0065 & 0.084 \\
& $(0.0087)$ & $(0.027)^{* * *}$ \\
\hline $\begin{array}{c}\text { Lag log of } \\
\text { labor } \\
\text { productivity }\end{array}$ & 0.0067 & -0.0068 \\
& $(0.0055)$ & $(0.00876)$ \\
\hline Lag log of age & -0.00437 & -0.01015 \\
& $(0.0027)$ & $(0.0036)^{* * *}$ \\
\hline Foreign owner \\
dummy & 0.0234 & 0.0605 \\
& $(0.00567)^{* * *}$ & $(0.0086)^{* * *}$ \\
\hline
\end{tabular}

*significant at $10 \%$; ${ }^{* *}$ significant at $5 \% ;{ }^{* * *}$ significant at $1 \%$

To understand the economic magnitude of the effects we report in Table 13 the 
marginal effect of the Heckman selection model calculated at the mean of each variable. Concentrating on the effect of exchange rates on export share, the table shows that adding 1 index point $(1995=100)$ to the REER will decrease export share by about 0.0034 percentage points, which is equivalent to a decrease of about 1.28 percent. ${ }^{16}$ As the REER index mainly changes between 3 and 10 index points each year, it therefore induces changes of export share between 5 and 13 percent at the mean. Big changes of REERs in some years may cause a change of 25 percent in export share at the mean, for example in 1995-1996. The evidence shows a higher negative exchange rate impact on export shares, compared with those of other studies from micro data such as Campa (2004), in which a 10 percent depreciation results in increases in export volume due to the increase in export intensity of 6.3 percent.

Effects of REER: foreign vs. domestic firms: We are also interested in the effects of exchange rate movements on different type of firms: foreign owned firms and domestic firms. To capture this we interact the foreign ownership dummy with the industry specific REER. The reference group is domestic firms. We only report the coefficients for REER, the product term and the foreign ownership dummy in Table 14, as our main concern is the interaction between the exchange rate and ownership dummy and the other independent variables have little effect when adding the interaction terms in the regression. The results in Column 1 show the interaction terms are both insignificant in the export intensity and export participation decisions, which suggests little difference between the two groups. However, we find that the coefficient on the foreign ownership dummy also

\footnotetext{
${ }^{16}$ This is computed using the mean of export share. From the estimates in table 12 the mean of export share is 0.2662 . So the change in percentage terms is $(0.0034 / 0.2662) 100=1.28$.
} 
becomes insignificant. We checked the correlation between the interaction terms and foreign ownership dummy, and found a correlation of more than 0.99 . There may be a problem of multicollinearity. To avoid this, we mean centre the REER index (subtract mean from REER), generate an interaction term between country owner dummy and the centered REER, and rerun the regression. ${ }^{17}$ The coefficients for country dummies in both equations become strongly significant again. However, this only changes the coefficients on foreign dummies.

Table 14: Heckman Selection Model: Foreign vs. Domestic Firms

\begin{tabular}{|c|c|c|c|c|c|c|c|c|}
\hline & \multicolumn{2}{|c|}{$\begin{array}{l}\text { (1) interacting with } \\
\text { foreign dummy }\end{array}$} & \multicolumn{2}{|c|}{$\begin{array}{l}\text { (2) quadratic } \\
\text { interacting with } \\
\text { foreign dummy* }\end{array}$} & \multicolumn{2}{|c|}{$\begin{array}{l}\text { (3) } \\
\text { foreign dummy=1 }\end{array}$} & \multicolumn{2}{|c|}{$\begin{array}{c}\text { (4) } \\
\text { foreign dummy }=0\end{array}$} \\
\hline & $\begin{array}{l}\text { Export } \\
\text { Dummy }\end{array}$ & $\begin{array}{l}\text { Export } \\
\text { Share }\end{array}$ & $\begin{array}{l}\text { Export } \\
\text { Dummy }\end{array}$ & $\begin{array}{l}\text { Export } \\
\text { Share }\end{array}$ & $\begin{array}{l}\text { Export } \\
\text { Dummy }\end{array}$ & $\begin{array}{l}\text { Export } \\
\text { Share }\end{array}$ & $\begin{array}{l}\text { Export } \\
\text { Dummy }\end{array}$ & $\begin{array}{l}\text { Export } \\
\text { Share }\end{array}$ \\
\hline $\begin{array}{c}\text { Lag } \\
\text { Industry } \\
\text { REER }\end{array}$ & $\begin{array}{l}0.0006 \\
(0.10)\end{array}$ & $\begin{array}{c}-0.004 \\
(-2.02) \\
* *\end{array}$ & $\begin{array}{l}-0.004 \\
(-0.62)\end{array}$ & $\begin{array}{c}-0.004 \\
(-1.93) \\
*\end{array}$ & $\begin{array}{l}0.0015 \\
(0.19)\end{array}$ & $\begin{array}{l}-0.003 \\
(-1.40)\end{array}$ & $\begin{array}{l}0.0025 \\
(0.34)\end{array}$ & $\begin{array}{c}-0.005 \\
(-2.47) \\
* *\end{array}$ \\
\hline $\begin{array}{l}\text { Foreign } \\
\text { owner } \\
\text { dummy }\end{array}$ & $\begin{array}{l}-0.278 \\
(-0.94)\end{array}$ & $\begin{array}{l}0.0552 \\
(1.24)\end{array}$ & $\begin{array}{c}0.128 \\
(3.22) \\
* * *\end{array}$ & $\begin{array}{c}0.0531 \\
(6.28) \\
* *\end{array}$ & & & & \\
\hline $\begin{array}{l}\text { InREER* } \\
\text { Foreign } \\
\text { dummy }\end{array}$ & $\begin{array}{l}0.0035 \\
(1.40)\end{array}$ & $\begin{array}{l}0.0000 \\
(0.07)\end{array}$ & $\begin{array}{l}0.0036 \\
(1.39)\end{array}$ & $\begin{array}{l}0.0003 \\
(0.57)\end{array}$ & & & & \\
\hline InREER $^{2}$ & & & $\begin{array}{c}0.001 \\
(2.07) \\
* *\end{array}$ & $\begin{array}{l}-0.000 \\
(-0.49)\end{array}$ & & & & \\
\hline $\begin{array}{c}\text { InREER }{ }^{2} \\
{ }^{*} \text { foreign } \\
\text { dummy }\end{array}$ & & & $\begin{array}{l}0.000 \\
(0.04)\end{array}$ & $\begin{array}{c}0.000 \\
(1.99) \\
* *\end{array}$ & & & & \\
\hline $\begin{array}{l}\text { Lambda } \\
\text { (std. } \\
\text { error) }\end{array}$ & \multicolumn{2}{|c|}{$\begin{array}{c}-0.0341 \\
(0.0056)^{* * *}\end{array}$} & \multicolumn{2}{|c|}{$\begin{array}{c}-0.0341 \\
(0.0056)^{* * *}\end{array}$} & \multicolumn{2}{|c|}{$\begin{array}{c}-0.0249 \\
(0.0105)^{* * *}\end{array}$} & \multicolumn{2}{|c|}{$\begin{array}{c}-0.0402 \\
(0.0059)^{* * *}\end{array}$} \\
\hline $\begin{array}{l}\text { Rho } \\
\text { (std. } \\
\text { error) }\end{array}$ & \multicolumn{2}{|c|}{$\begin{array}{c}-0.1332 \\
(0.0209)^{* * *}\end{array}$} & \multicolumn{2}{|c|}{$\begin{array}{c}-0.1332 \\
(0.0208)^{* * *}\end{array}$} & \multicolumn{2}{|c|}{$\begin{array}{c}-0.0924 \\
(0.0388)^{* * *}\end{array}$} & \multicolumn{2}{|c|}{$\begin{array}{c}-0.167 \\
(0.0245)^{* * *}\end{array}$} \\
\hline $\begin{array}{l}\text { No. of } \\
\text { obs. }\end{array}$ & \multicolumn{2}{|c|}{44,251} & \multicolumn{2}{|c|}{44,251} & \multicolumn{2}{|c|}{20,572} & \multicolumn{2}{|c|}{23,679} \\
\hline
\end{tabular}

Notes for Table 14 - Table 16, see notes for Table 12 .

* The REER index used in Column 2 is mean centered.

${ }^{17}$ See Wooldridge (2005) P235 for the method. 
As stated in Jaccard and Turrisi (2003), the traditional way to detect interaction effect using product terms only tests for an interaction with a specific form, i.e. simple linear relationship between the two variables. The traditional interaction models assuming simple linear interactions are too restrictive; other forms of interaction may exist but cannot be detected by them. So the possibility of curvilinear effects should be accommodated. One way of doing this is to allow for quadratic relationships between REER and foreign ownership dummy. ${ }^{18}$ So we generate two new independent variables: squared mean centered REER and the product of the squared REER and the foreign ownership dummy, and add them into our original interaction model to test for nonlinear interaction effects between the exchange rate and firm ownership. The reference group is still domestic firms. Column 2 in Table 14 reports the results for the quadratic interaction effect. The coefficient for the product term between squared REER and foreign dummy is positive and significant in export share equation, whereas the coefficient on the squared REER is insignificant. The positive and significant coefficient for the quadratic interaction term in export intensity shows there are strong nonlinear interaction effects between REER and foreign ownership dummy: foreign firms are less negatively affected by REER changes than domestic firms. The results suggest statistically significant differences between foreign firms and indigenous firms in the effects of changes in exchange rate level on firms' export intensity decision. It is possible that the significant coefficient for the squared interaction term is driven by outliers other than the nonlinear relationship we mentioned above. To check this, we use the method of Hadi $(1992,1994)$ to identify multiple outliers in multivariate

\footnotetext{
${ }^{18}$ To test a quadratic interaction effect between a continuous variable $X$ and a dummy variable $Y$, the following model is used: $Z=\alpha_{0}+\alpha_{1} X+\alpha_{2} Y+\alpha_{3} X Y+\alpha_{4} X^{2}+\alpha_{5} X^{2} Y+\varepsilon_{1}$, where $X$ is mean centered. The coefficient $\alpha_{5}$ represents the quadratic interaction effect.
} 
data. We find 186 outliers in our data. Results after dropping those outliers remain unchanged, which means that outliers do not affect our results. So the significant coefficient does imply significant differences between foreign and indigenous firms.

Table 15: Heckman Selection Model: Big vs. Small Firms

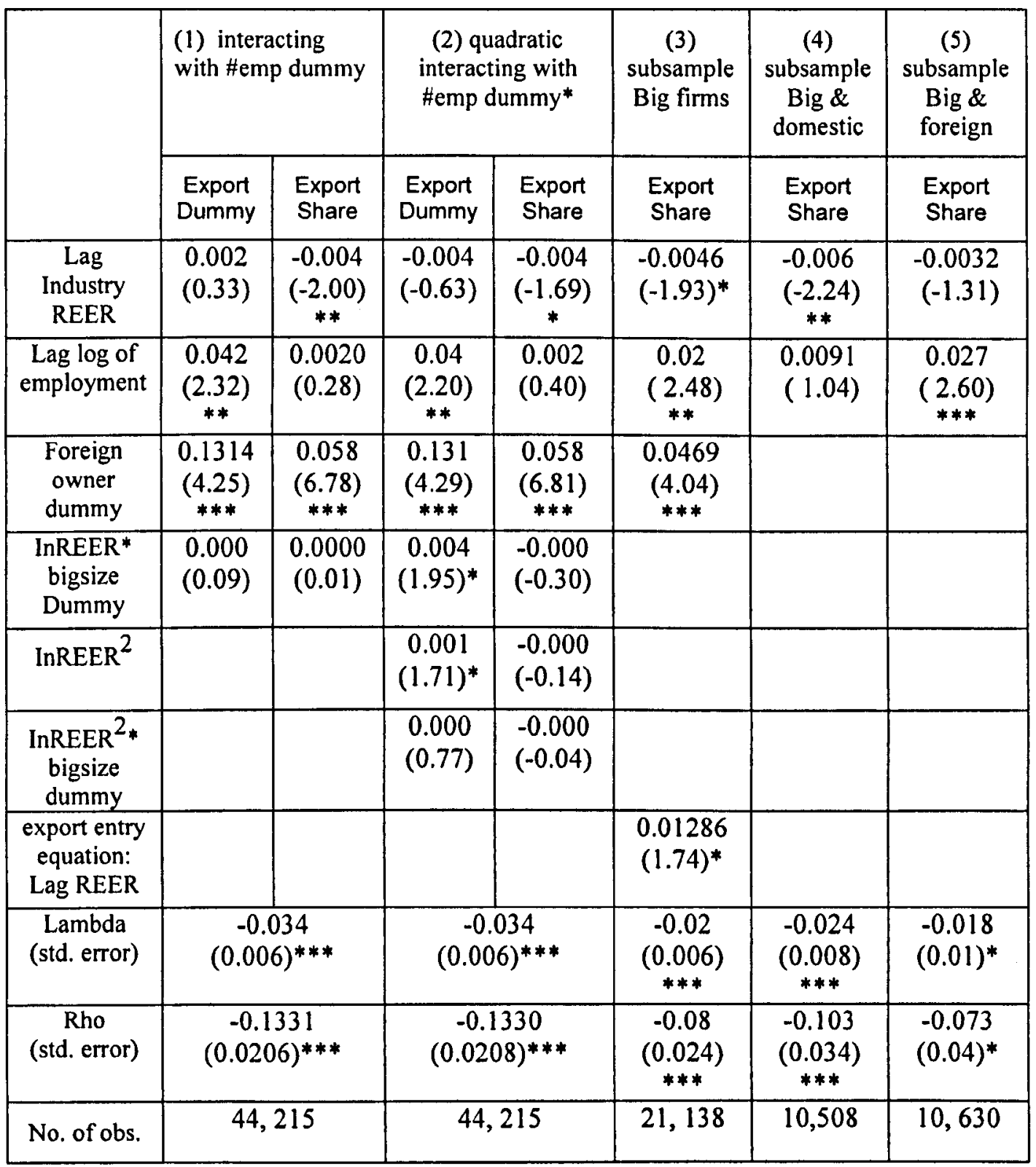

* The REER index used in Column 2 is mean centered.

An alternative approach to dealing with this is to estimate the sample selection model separately within the two subsamples. Columns 3 and 4 report the results of doing so. Column 3 shows the results for foreign owned firms. The coefficients of 
the exchange rate in the export share equation become insignificant albeit with expected signs. The results in Column 4 for domestic firms show that exchange rate changes have more significant effects on export shares than those in Table 12. Exchange rates have little impact on firm export participation decisions in both cases. The results are consistent with the idea that exchange rate changes have less impact on multinationals due to their ways of dealing with exchange rate risk. Although the method of subgroup comparison by calculating separate regressions for each group is not a standard way to test interaction effects, it provides significant supportive evidence for the hypothesis of the different effects.

Different effects of REER for different ownership types may be due to other factors such as size and country of origin. Size is the best and most obvious discriminator to use. As pointed out in some papers on financial factors such as Greenaway, Guariglia and Kneller (2007), size has been extensively used as a proxy for financial constraints faced by firms. It plays some role in affecting the firm's ability to finance export market entry and impact of macro shocks. Big firms are less likely to face financial constraints and less likely to be influenced by shocks. As a robustness check, we examine the effects of REER on big/small firms, using number of employees to separate two groups by the median of size. We interact the large size dummy with REER and include the interaction terms in the Heckman selection model. Column 1 of Table 15 reports our results. Size does not seem to matter under the assumption of a linear interaction: the coefficients of the interaction terms are both insignificant. We also interact the continuous variable of number of employees with the REER index, and the product terms in both equations are insignificant. 
As before, we then test the interaction effects for a nonlinear relationship by adding two more variables: squared mean centered REER and the product of the squared REER and the large size dummy. Column 2 shows that the quadratic interaction terms in both equations are always insignificant, which provides no significant evidence for the nonlinear difference of exchange rate effects on exports for firms of different size. Although this time the linear interaction term in export entry equation becomes positive and weakly significant, it does not contradict the argument that the different effects of the exchange rate on exports for different ownerships are not due to differences in size. We also separately examine the effects of REER for subsamples of firms. The results are shown in Columns 3, 4 and 5. Column 3 shows that the effects of REER on big firms are both weakly significant in export intensity and export entry decisions (negative in the former and positive in the latter), whereas those on small firms are not, which may be not consistent with the hypothesis of financial constraints in export intensity decisions but may be in export participation. However, it suggests that the insignificant effects of REER in export intensity equation found in Table 14 are not due to size but to ownership. Further splitting big firms into foreign and domestic, we find the coefficient for foreign firms is insignificant and significant for domestic big firms in Columns 4 and 5 . We only report the results for export share decisions since those on export participation are always insignificant except the subsample of big firms which is reported in the fourth last row in Column 3. This confirms the role of ownership. Splitting small firms by ownership, the coefficients are all insignificant. $^{19}$

\footnotetext{
${ }^{19}$ Real sales is an alternative proxy for size. For our sample, the differences in sales between foreign and domestic firms are much bigger than those in the number of employees. So we then interact sales dummy with REER, and also separate firms into two groups by the median of real sales to
} 
the coefficients for the 2 digit level industry dummies are strongly significant, which raises the question of whether there is any industry heterogeneity in effects of exchange rate changes on exports. This is another interaction effect we can detect. However, this time the qualitative variable is not binary, but twenty-two 2 digit manufacturing industries. To examine the interaction effect between exchange rate and 2 digit level industry, we can choose an industry as the reference group, generate 21 industry dummies for the rest of industries, and interact each of the industry dummies with REER. ${ }^{20}$ Adding the 21 product terms, we run the regression and get the coefficients for the product terms to see if there are significant difference between the reference industry and each of the rest of industries.

For 22 industries, we will have a $22 * 22$ matrix. For brevity, we just report the sign and significance of the interaction terms in Column 1 of Table 16, using Industries 22,29 , and 36 as the reference. The results are typical of industry heterogeneity in the effects of exchange rate on exports. Subcolumn (a) reports the slope difference between Industry 22 and the other industries: only 4 out of 21 industries have insignificant coefficients in export share equation, which means that the effects of

check the effects of REER. The results are similar to those in Table 15: size does not matter. Results suggest that the difference in the effects of REER we find between domestic and foreign firms comes mainly from the different ownership of firms rather than the different firm size.

${ }^{20}$ For example, the method to test interaction effect between a continuous variable $\mathrm{X}$ and a qualitative variable $\mathrm{D}$ with 3 levels (level $\mathrm{a}, \mathrm{b}$, and $\mathrm{c}$ ), is to generate two dummies $D b$ and $D_{c}$ (level a as the reference), the following is estimated: $Z=\alpha_{0}+\alpha_{1} X+\alpha_{2} D_{b}+\alpha_{3} D_{c}+\alpha_{4} X D_{b}+\alpha_{5} X D_{c}+\varepsilon_{1}$. The coefficients $\alpha_{4}$ and $\alpha_{5}$ reflect the difference in slopes of $Z$ on $X$ between level $a$ and $b$ and between $a$ and $c$ respectively. If one wants to evaluate the contrast comparing the slope for $b$ versus $c$, the contrast and its relevant significance tests can be done by changing the reference group to either level $b$ or level $c$ and creating a new set of dummies and product terms, then rerunning the regression and examining the coefficients for the interaction terms. For qualitative variable with more than 3 levels, the process is curbersome. 
exchange rate on export intensity for 17 industries are significantly different from that on Industry 22 . The effects in export entry decision are a bit different: there are fewer industries ( 10 industries) with different exchange rate effects for export entry, compared to that for Industry 22. Subcolumn (b) shows that 8 industries are significantly different from Industry 29 in the effects of exchange rate on export intensity decisions, whereas 14 are different in the export entry equation. Subcolumn (c) also shows a similar picture: some industries incur significantly different effects of exchange rate on both export entry and export intensity decisions, with Industry 36 as the reference industry.

We also split our sample into 22 subgroups by 2 -digit level industries, and separately run the sample selection model within each to estimate the coefficients of REER. Column 2 in Table 16 reports the sign and significance of REER on export decisions for each 2-digit industry. We observe significant effects on export entry for 7 industries. 7 out of 16 industries are insignificant both in export participation and export share decisions. There are 4 industries with significantly negative coefficients for either export entry or export share decision or both, and 4 industries with significantly positive coefficients. Table 17 reports the summary of the effects reported in Column 2 of Table 16 across 2-digit industries with detailed industry names, in which the industries are split into 6 categories according to different effects in export entry and export intensity decisions. We list negative effect categories at the top, the insignificant effect category in the middle, and the positive effect categories at the bottom of the table. The different effects of exchange rate level changes on exports across industries cannot be easily explained 
by just superficially looking at the names of the industries. This table can be used

to make some comparisons later in the thesis.

Table 16: Industry Heterogeneity: Level of REER on Exports

\begin{tabular}{|c|c|c|c|c|c|c|c|c|c|}
\hline \multirow[t]{3}{*}{$\begin{array}{l}\text { Industry } \\
\text { Code }\end{array}$} & \multicolumn{6}{|c|}{$\begin{array}{l}\text { (1) Interaction term } \\
\text { (centered REER*industry dummy) }\end{array}$} & \multirow{2}{*}{\multicolumn{3}{|c|}{$\begin{array}{c}\text { (2) REER } \\
\text { ( for subsample of each } 2 \\
\text { digit level industry) }\end{array}$}} \\
\hline & \multicolumn{2}{|c|}{ (a) Ref. Ind.:22 } & \multicolumn{2}{|c|}{ (b) Ref. Ind.:29 } & \multicolumn{2}{|c|}{ (c) Ref. Ind.:36 } & & & \\
\hline & $\begin{array}{l}\text { Export } \\
\text { dummy }\end{array}$ & $\begin{array}{l}\text { Export } \\
\text { share }\end{array}$ & $\begin{array}{l}\text { Export } \\
\text { dummy }\end{array}$ & $\begin{array}{l}\text { Export } \\
\text { share }\end{array}$ & $\begin{array}{l}\text { Export } \\
\text { dummy }\end{array}$ & $\begin{array}{c}\text { Export } \\
\text { share }\end{array}$ & $\begin{array}{l}\text { Export } \\
\text { dummy }\end{array}$ & $\begin{array}{c}\text { Export } \\
\text { share }\end{array}$ & \#Obs. \\
\hline 15 & + & - & $+* *$ & $-*$ & - & $-* * *$ & + & - & 1830 \\
\hline 16 & $-* * *$ & $+* * *$ & $-* * *$ & $+* * *$ & -*** & $+* * *$ & $\mathrm{~N} / \mathrm{A}$ & & \\
\hline 17 & $+* *$ & $+* * *$ & $+* * *$ & + & + & - & - & - & 1271 \\
\hline 18 & - & - & $+* *$ & $-* *$ & $-* * *$ & $-* * *$ & + & + & 641 \\
\hline 19 & + & $+* * *$ & + & $+* * *$ & + & $+* * *$ & N/A & & \\
\hline 20 & - & + & + & - & - & - & - & + & 344 \\
\hline 21 & $+* * *$ & $+* * *$ & $+* * *$ & - & - & $-* * *$ & N/A & & \\
\hline 22 & N/A & N/A & $+* *$ & $-* *$ & $-* * *$ & $-* * *$ & $-* * *$ & $-* * *$ & 6503 \\
\hline 23 & $+* *$ & $+* * *$ & $+* * *$ & + & - & - & N/A & & \\
\hline 24 & $+* * *$ & $+* * *$ & $+* * *$ & + & + & + & - & + & 2699 \\
\hline 25 & $+* *$ & $+* * *$ & $+* * *$ & + & - & - & N/A & & \\
\hline 26 & + & + & + & - & - & $-* *$ & + & $\overline{-}$ & 1776 \\
\hline 27 & + & $+* * *$ & $+^{*}$ & + & $-* *$ & - & $-* *$ & $-*$ & 1486 \\
\hline 28 & + & $+* * *$ & $+* *$ & + & - & - & - & $-* * *$ & 4808 \\
\hline 29 & $-* *$ & $+* *$ & $\mathrm{~N} / \mathrm{A}$ & $\mathrm{N} / \mathrm{A}$ & $-* * *$ & $-*$ & + & - & 5856 \\
\hline 30 & $+* *$ & $+* * *$ & $+* * *$ & $+* *$ & - & $+* *$ & N/A & & \\
\hline 31 & + & $+* * *$ & + & $+* *$ & - & + & - & + & 1008 \\
\hline 32 & $+* *$ & $+* *$ & $+* * *$ & + & - & + & $+* *$ & $+* * *$ & 1528 \\
\hline 33 & - & $+* *$ & + & - & $-* * *$ & $-* * *$ & $+^{* *}$ & -**** & 720 \\
\hline 34 & - & $+*$ & + & + & $-* * *$ & + & $+* *$ & + & 1736 \\
\hline 35 & - & $+^{*}$ & - & + & - & - & $-* * *$ & - & 326 \\
\hline 36 & $+* * *$ & $+* * *$ & $+* * *$ & $+^{*}$ & $\mathrm{~N} / \mathrm{A}$ & N/A & $+* * *$ & + & 5553 \\
\hline
\end{tabular}

* indicates significant at $10 \% ;{ }^{* *}$ indicates significant at $5 \% ;{ }^{* *}$ indicates significant at $1 \%$

Generally, Tables 16 and 17 shows strong evidence for industry heterogeneity in

exchange rate effects. This is consistent with Das, Roberts, and Tybout (2007) who also find significant cross-industry variation in the effects of exchange rate 
movements. However, the existing theoretical literature does not provide any clear guidance in explaining heterogeneity.

\section{Table 17: Summary of Industry Heterogeneity in Effects of Exchange Rate Level}

\begin{tabular}{|c|c|}
\hline $\begin{array}{l}\text { Different } \\
\text { effect type }\end{array}$ & Industries \\
\hline $\begin{array}{l}\text { export entry } \\
\text { (negative), } \\
\text { export share } \\
\text { (insignificant } \\
\text { or negative) }\end{array}$ & $\begin{array}{l}22 \text { Publishing, Printing and Reproduction of Recorded Media } \\
27 \text { Manufacture of Basic Metals } \\
35 \text { Manufacture of Other Transport Equipment }\end{array}$ \\
\hline $\begin{array}{l}\text { export entry } \\
\text { (insignificant) } \\
\text { export share } \\
\text { ( negative) } \\
\end{array}$ & $\begin{array}{l}28 \text { Manufacture of Fabricated Metal Products, Except } \\
\text { Machinery and Equipment }\end{array}$ \\
\hline $\begin{array}{l}\text { Insignificant in } \\
\text { both decisions }\end{array}$ & $\begin{array}{l}15 \text { Manufacture of Food Products and Beverages } \\
17 \text { Manufacture of Textiles } \\
18 \text { Manufacture of Wearing Apparel; Dressing and Dyeing of Fur } \\
20 \text { Manufacture of Wood And Products of Wood And Cork, Except } \\
\text { Furniture; Manufacture of Articles of Straw and Plaiting Materials } \\
24 \text { Manufacture of Chemicals and Chemical Products } \\
26 \text { Manufacture of Other Non-metallic Mineral Products } \\
29 \text { Manufacture of Machinery and Equipment Not Elsewhere } \\
\text { Classified } \\
\text { 31 Manufacture of Electrical Machinery and Apparatus Not } \\
\text { Elsewhere Classified }\end{array}$ \\
\hline $\begin{array}{l}\text { export entry } \\
\text { (positive) } \\
\text { export share } \\
\text { (negative) }\end{array}$ & $\begin{array}{l}33 \text { Manufacture of Medical, Precision and Optical Instruments, } \\
\text { Watches and Clocks }\end{array}$ \\
\hline $\begin{array}{l}\text { export entry } \\
\text { (positive) } \\
\text { export share } \\
\text { (insignificant } \\
\text { or positive) } \\
\end{array}$ & $\begin{array}{l}32 \text { Manufacture of Radio, Television and Communication } \\
\text { Equipment and Apparatus } \\
34 \text { Manufacture of Motor Vehicles, Trailers and Semi-trailers } \\
36 \text { Manufacture of Furniture; Manufacturing Not Elsewhere } \\
\text { Classified }\end{array}$ \\
\hline
\end{tabular}

\section{Conclusions}

This chapter examined the effects of exchange rate movements on firm decisions 
on export entry, exit and export share. The analysis breaks down export adjustments between changes in export share by existing exporters and those due to changes in entry into and exit from export markets. Using data on a sample of UK manufacturing firms, we find evidence for the presence of sunk costs. Results show that firm export participation and exit decisions are not strongly related to exchange rate movements. The exchange rate has a significant and negative impact on the export share of firms after entry. The responsiveness of export share on the degree of exchange rate changes is not quantitatively as small as in Campa (2004). One index point depreciation in REER index will increase export share by about 1.28 percent. Generally, the evidence suggests that export adjustments due to exchange rate changes mainly occur through export share by existing exporters rather than changes in the number of exporting firms.

We also find the export behavior of multinational firms is less likely to be affected by exchange rate changes than that of non-multinationals. The results provide the first direct evidence for the hypothesis of multinationals' ability to deal with exchange rate risk. In the end, we showed that industry heterogeneity is important in the effects of exchange rate on exports. For some industries, the effects of exchange rate changes on exports are negative, for some, the effects are insignificant or positive. Ignoring heterogeneity can mean questions being posed against evidence. If there is industry heterogeneity in the effects, evidence from macro data may suffer from an aggregation problem. This might partly explain why evidence from macro data is mixed whereas that from micro data is not. So an interesting direction for future research is to try to explain industry heterogeneity. We will propose two hypotheses to do so in the next two chapters. 
Appendix: Definitions of the Variables Used

Export dummy: dummy variable equal to 1 if the firm's overseas turnover is positive

Real intangible assets: the firm's intangible assets deflated by RPI indices (Source: Office of National Statistics)

Real Sales: includes both UK and overseas turnover deflated by PPI indices (Source: Office of National Statistics)

Labor productivity: the ratio of the firm's total real sales to its total number of employees.

Real Wage: the ratio of the firms' total wage bill (which includes wages, salaries, social security and pension costs) to number of employees, deflated by RPI indices. Foreign owner dummy: dummy variable equal to 1 if the firm's primary ownership country is not $\mathrm{UK}$, and 0 otherwise. This variable is only available in the last year of observations available for each firm. We therefore have to assume that a firm which was foreign owned in its last available year was foreign owned throughout the period in which it was observed.

Log of employment: Number of employees

Export Share: ratio between overseas turnover and total turnover Age: the subtraction of current year and the incorporation year for each firm in each year

Industry specific REER: 3-digit manufacturing industry level real effective exchange rate 


\section{Chapter 3}

\section{Explaining Industry Heterogeneity: Controlling for}

\section{External Orientation}

\section{Introduction}

In Chapter 2, we showed that the effects of changes in exchange rate level on export decision of firms are different across industries, when we had controlled for firm characteristics. In this chapter, we aim to explain industry heterogeneity by test a hypothesis on the cost impact of exchange rate changes.

To understand how export behaviour in an industry is affected by international shocks, such as exchange rate movements, we need to investigate the role of the degree of exposure to external shocks ${ }^{1}$ of the industry. Real exchange rate appreciation not only makes the foreign export price more expensive, it also makes imported inputs cheaper, which may lead to cost reduction. Thus the net effects of an exchange rate change on export prices and production costs are not therefore so obvious. If induced cost changes are significant, the negative effects of exchange rate changes on exports may be upward biased. Since international trade in intermediate inputs is becoming more and more important, we cannot ignore the effect of exchange rate movement on firms' production costs. In the previous chapter, we only considered the effects of exchange rate changes on exports, and ignored those on imported intermediate inputs and their indirect role on exports. In this chapter, we will take the cost impact of exchange rate changes into

\footnotetext{
' In Campa and Goldberg (1997a), the degree of exposure to external shocks is called external orientation. We follow them in this chapter.
} 
consideration. So the study on the relationship between exchange rate changes and exports should also require basic knowledge on the level and type of external orientation for a particular industry.

Several papers look at the role of external orientation. Using data for US industries, Goldberg (1993) finds that US industry investment activities under exchange rate appreciation are sensitive to the magnitude of industry external orientation. Using two decades annual data for U.S. manufacturing industries, Campa and Goldberg (2001) argue that industry external orientation has significant effects on the degree of industry employment response to exchange rates. Using data for 21 OECD countries, Campa and Goldberg (2006) find that imported intermediate inputs is a key factor to influence the degree of exchange rate pass-through across countries. Athukorala and Menon (1994) study exchange rate pass-through in Japanese exports, and find that "the cost lowering effect of exchange rate changes seems to have provided Japanese exporters with considerable leverage in enduring the massive yen appreciation". They also suggest that industry variation in the degree of pass-through may reflect differences in cost conditions across industries, and disaggregate data are needed to generate more meaningful studies. Since each industry has a different degree of external orientation, we may expect this partly explain the industry heterogeneity in the effects of exchange rate changes on exports.

There does not exist a single measure of industry external orientation. An ideal measure should reflect differences across industries and over time in industry sensitivity to external forces like exchange rate movements, and should be specific 
to the particular subject of exposure (Campa and Goldberg, 1997). At present, the most widely used indicator is industry openness to trade, calculated as value of the industry trade (exports and imports) in final goods relative to domestic production or consumption in the industry. However, this measure ignores other channels of external orientation that have growing importance, including the increasing role of imported inputs into production and various components of multinational activity. An industry with a low level of "openness to trade" could have significant exposure to external shocks if it relies heavily on imported goods as inputs or if it engages in FDI. So a good measure of external exposure to exchange rate shocks should include at least two main aspects: exports of final goods and imports of intermediate inputs, which we refer to as outward orientation and inward orientation.

Chapter 2 used export weighted real effective exchange rates to investigate the relationship between exchange rate movements and exports. This has already embodied outward orientation. There are two main ways to control for external orientation: one is to add measures of inward orientation, i.e. imported intermediate inputs, in our previous outward oriented export weighted REER related regressions; the other is to generate new indicators which take both outward orientation and inward orientation into consideration and to substitute the new indicators for our previous outward oriented export weighted REER in the regressions. We use both approaches.

The structure of this chapter is as follows: the next section focuses on measuring external orientation and summary statistics; Section 3 presents the results after 
controlling for external orientation in two ways; Section 4 concludes.

\section{Measures of External Orientation and Summary Statistics}

\subsection{Classification of measures and hypotheses}

We rely on two families of indicators for each 2-digit manufacturing industry to proxy for the inward orientation (Family 1), and net external orientation, combining inward orientation with outward orientation (Family 2).

To evaluate the impact of the inward orientation for each 2-digit manufacturing industry, we will use two measures of inward orientation (Family 1), one based on imported input share alone, and the other on both imported input share and imported input related real effective exchange rates (REER). Specifically:

(1) The ratio of imported intermediate inputs relative to total intermediate inputs in producing goods in each 2-digit output industry (IMPORT RATIO), which measures the level of inward orientation.

(2) The imported intermediate input REER (III REER), which is generated not only from imported input ratios but also from the exchange rate movements of the currencies in countries from which imports originate relative to sterling. This assumes that exchange rate changes are highly passed-through into imported goods prices and thus change the production costs of firms. The second measure is novel and is, to our knowledge, the first attempt to account for the effects of imported related REER changes. Appendix 1 introduces the method to calculate III REER.

To evaluate the net effects of external orientation, combining inward orientation 
with outward orientation simultaneously for each 2-digit manufacturing industry, we rely on the following three measures of net external orientation (Family 2):

(3) Net external ratio (NET RATIO), calculated as the difference between export revenues relative to total domestic output and the imported intermediate input ratio (i.e. indicator (1) in Family 1). This follows Campa and Goldberg (1997), which takes the offset effects of exports and imports into account. ${ }^{2}$ Although this measure includes both export and import roles, the differences in exchange rate movements between export-related products and imported intermediate inputs are ignored.

(4) Net REER (NET REER), calculated simply as the product of export REER and export share (export revenues relative to total domestic output) minus the imported-intermediate REER (i.e. indicator (2) in Family 1). This is the simplest way to incorporate outward orientation with inward orientation to calculate new REER indices, which takes changes both in exported output related REER and in imported inputs related REER into consideration.

(5) Effective external orientation index (EFFECT INDEX), is a more complex index. The effective index borrows the method in computing effective protection in trade policy (see for example in Greenaway and Milner, 1993). The main idea is to generate an index to represent changes in value added due to exchange rate changes within an industry. For each industry, there are imported intermediate inputs and exported outputs that incur the impacts from exchange rate changes. The measure is trying to include all these impacts simultaneously in a fairly simple way. The method to obtain EFFECT INDEX is detailed in Appendix 2.

\footnotetext{
${ }^{2}$ See Campa and Goldberg (1999) and Campa and Goldberg (1995) for detailed model of exposure to generate the net exposure share.
} 
To our knowledge, the last two measures are the first to include imported intermediate input ratio, export share, export related REER and import related REER to generate net and effective indices due to exchange rate changes within a given industry.

The two measures in Family 1 consider only inward aspect of external orientation, which can be interacted respectively with export weighted REER in regressions to see whether an industry with a high degree of inward orientation would incur less negative impact from export REER than industry with a low degree of inward orientation. In this way, we account for both aspects of external orientation. And we expect that there are offsetting effects between the inward orientation measures and the effects of export REER on exports. The intuition behind this hypothesis is that exchange rate movements move the export price and imported inputs price in opposite directions.

Since measure (3), NET RATIO, indicates the net export share (i.e. net outward ratio) within an industry, if it is interacted with export related REER, we expect that there may be a reinforcing effect on the role of export REER. The intuition here is that the higher the net ratio, the more the industry is exposed to export related exchange rate impacts, and the stronger the effects of exported weighted REER on export decisions of firms. However, interacting NET RATIO with export REER may not be a good way to evaluate the role of external orientation, as export REER represents solely the outward orientation disregarding the inward side, whereas NET RATIO is just a trade related index including both import and export. If there is an interaction effect between the two, it is hard to tell whether it implies 
the role of external orientation, or the role of market power (represented by the trade related index) or something else.

The last two measures (4) (5), which have already taken both export and import related exchange rate movements, as well as outward and inward ratio, into consideration, cannot be put simultaneously with export related REER into regressions. So we substitute the two indicators for our previous outward oriented export REER in the regressions. We expect that the effects of net and effective indices on export behavior may be weaker than those of export REER due to offsetting effects between inward and outward orientation.

\subsection{Data sources, descriptive statistics and correlations}

To be consistent with other variables in our study, measures of external orientation should cover the period of $1988-2004$. Due to the availability of data from inputoutput tables, we calculate the measures for each 2-digit manufacturing industry instead of more disaggregated levels such as 3-digit industry level.

IMPORT RATIO is calculated from the annual input-output tables for the UK published by the ONS. The available input-output tables to compute the ratio are available only for three years: 1990,1995 and $1998 .^{3}$ The resulting gaps are imputed by linear extrapolation. The imported intermediate inputs we used to compute the ratio include both goods and services. Table 1 shows the IMPORT RATIO for each 2-digit manufacturing industry in 1990, 1995 and 1998. More than

\footnotetext{
${ }^{3}$ IO tables for 1990 and 1995 are from ONS, IO table for 1998 is from OECD. In the table for 1998, some industries are aggregated together. So we have to guess the value according to its previous pattern when we do linear regression to fill up the missing values.
} 
half of industries change little between 1990 and 1998. The average share is $29.3 \%$ in $1998,29.5 \%$ in 1995 and $30.9 \%$ in 1990 (excluding industry 16). About half of the industries have a quite stable IMPORT RATIO across the period. Industries with significant decreasing ratios are $18,21,23,25$, whereas the ratios for 26,29 , 30,35 rise significantly.

Table 1: IMPORT RATIO for Each 2-digit Manufacturing Industry

\begin{tabular}{|c|c|c|c|}
\hline Industry & 1990 & 1995 & 1998 \\
\hline 15 & $16.91 \%$ & $15.95 \%$ & \multirow{2}{*}{$19.35 \%$} \\
\hline 16 & $39.13 \%$ & $9.83 \%$ & \\
\hline 17 & $44.44 \%$ & $34.28 \%$ & \multirow{2}{*}{$35.91 \%$} \\
\cline { 1 - 3 } 18 & $52.22 \%$ & $41.61 \%$ & \\
\hline 19 & $27.91 \%$ & $27.81 \%$ & \\
\hline 20 & $30.95 \%$ & $23.21 \%$ & $24.52 \%$ \\
\hline 21 & $39.25 \%$ & $36.94 \%$ & \multirow{2}{*}{$21.45 \%$} \\
\hline 22 & $23.28 \%$ & $23.44 \%$ & \\
\hline 23 & $37.88 \%$ & $21.44 \%$ & $13.73 \%$ \\
\hline 24 & $31.08 \%$ & $31.92 \%$ & $29.36 \%$ \\
\hline 25 & $37.71 \%$ & $28.05 \%$ & $27.01 \%$ \\
\hline 26 & $14.37 \%$ & $18.83 \%$ & $24.38 \%$ \\
\hline 27 & $28.98 \%$ & $27.61 \%$ & $31.36 \%$ \\
\hline 28 & $21.53 \%$ & $21.87 \%$ & $24.23 \%$ \\
\hline 29 & $20.28 \%$ & $24.53 \%$ & $26.13 \%$ \\
\hline 30 & $36.00 \%$ & $48.95 \%$ & $54.15 \%$ \\
\hline 31 & $27.42 \%$ & $27.31 \%$ & $31.95 \%$ \\
\hline 32 & $35.25 \%$ & $38.49 \%$ & $38.62 \%$ \\
\hline 33 & $26.80 \%$ & $29.65 \%$ & $31.23 \%$ \\
\hline 34 & $36.48 \%$ & $32.54 \%$ & $31.72 \%$ \\
\hline 35 & $32.11 \%$ & $38.52 \%$ & $37.83 \%$ \\
\hline 36 & $27.29 \%$ & $26.18 \%$ & $24.86 \%$ \\
\hline
\end{tabular}

Appendix 1 explains the method and data sources for computing III REER. Figure 1 shows the patterns of III REER. ${ }^{4}$ They are different across industry and time due to different imported input ratios across industry and import REER across industry and time. Table 2 shows the summary statistics. Industry 15 has the lowest average

\footnotetext{
${ }^{4}$ The abrupt trend break in Figure 1 for Industry 16 is due to using different values of data from input-output table for different time periods: 1988-1994 and 1995-2004. See Appendix 1 for details. However, Industry 16 is a small industry. So we don't worry too much about the break.
} 
index of 24.5 and is the most stable industry with the smallest standard deviation. Industry 18 has the highest average index of 71.4. Differences among the industries are quite large, which may due to differences in imported input ratios as well as import REER.

Figure 1: III REER
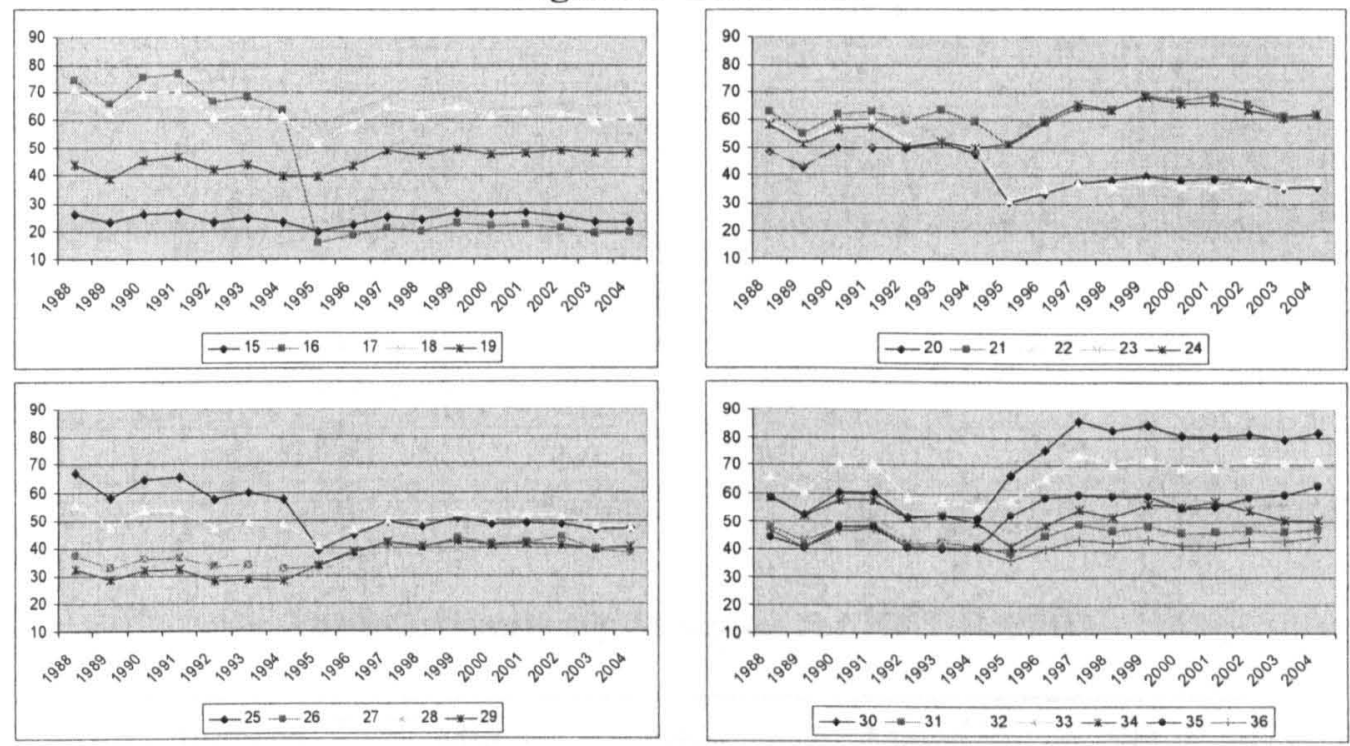

Table 2: Summary Statistics for III REER

\begin{tabular}{cccc}
\hline Industry & Mean & Median & SD \\
\hline 15 & 24.51 & 24.70 & 1.84 \\
16 & 40.78 & 22.36 & 25.42 \\
17 & 62.69 & 62.05 & 4.64 \\
18 & 71.43 & 68.05 & 9.60 \\
19 & 45.21 & 46.73 & 3.57 \\
20 & 41.49 & 38.84 & 6.64 \\
21 & 61.87 & 62.69 & 4.64 \\
22 & 43.84 & 37.74 & 10.05 \\
23 & 38.10 & 32.78 & 11.17 \\
24 & 58.80 & 59.47 & 6.13 \\
25 & 52.98 & 49.29 & 8.05 \\
26 & 37.74 & 38.06 & 3.92 \\
27 & 49.80 & 49.85 & 3.59 \\
28 & 35.92 & 36.75 & 2.50 \\
29 & 35.94 & 38.87 & 5.66 \\
30 & 69.71 & 75.79 & 13.39 \\
31 & 45.30 & 46.27 & 2.98 \\
32 & 66.42 & 69.07 & 6.12 \\
33 & 51.36 & 53.71 & 5.28 \\
34 & 52.76 & 52.19 & 4.36 \\
35 & 51.77 & 54.78 & 8.18 \\
36 & 42.36 & 42.35 & 2.78 \\
\hline
\end{tabular}


Figure 2: NET RATIO for Each 2-digit Manufacturing Industry
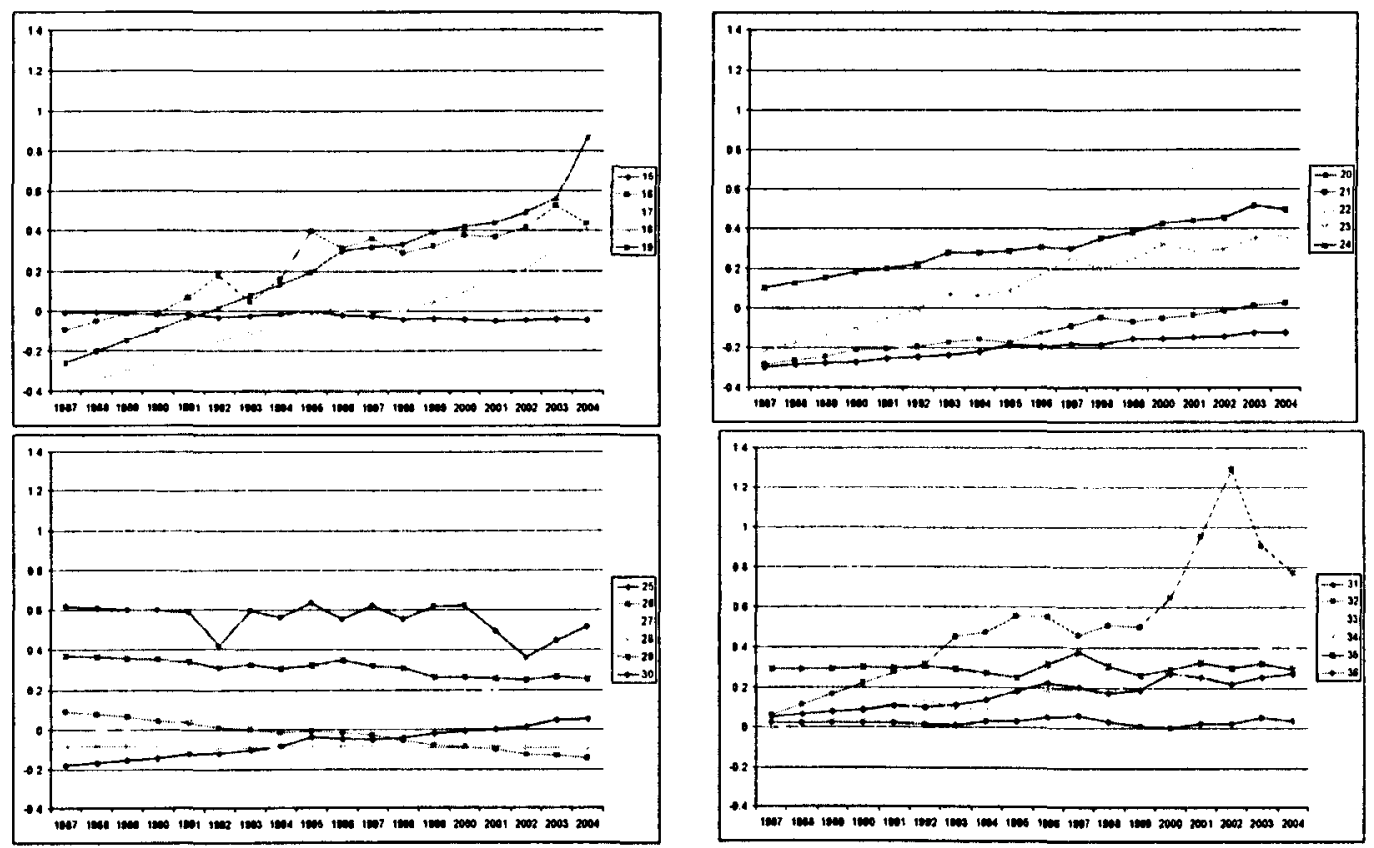

Table 3: Summary Statistics for NET RATIO

\begin{tabular}{ccccc}
\hline Industry & Min & Max & Mean & SD \\
\hline 15 & -0.050 & 0.001 & -0.027 & 0.016 \\
16 & -0.096 & 0.523 & 0.225 & 0.195 \\
17 & -0.222 & 0.248 & -0.013 & 0.133 \\
18 & -0.381 & 0.411 & -0.034 & 0.222 \\
19 & -0.260 & 0.864 & 0.210 & 0.301 \\
20 & -0.299 & -0.120 & -0.206 & 0.059 \\
21 & -0.285 & 0.024 & -0.129 & 0.099 \\
22 & -0.153 & -0.073 & -0.117 & 0.024 \\
23 & -0.219 & 0.364 & 0.111 & 0.190 \\
24 & 0.104 & 0.518 & 0.306 & 0.128 \\
25 & -0.182 & 0.053 & -0.066 & 0.074 \\
26 & -0.148 & 0.090 & -0.027 & 0.074 \\
27 & -0.021 & 0.329 & 0.118 & 0.097 \\
28 & -0.095 & -0.079 & -0.088 & 0.005 \\
29 & 0.247 & 0.369 & 0.309 & 0.041 \\
30 & 0.357 & 0.634 & 0.555 & 0.079 \\
31 & 0.051 & 0.270 & 0.164 & 0.075 \\
32 & 0.059 & 1.293 & 0.512 & 0.318 \\
33 & 0.219 & 0.411 & 0.305 & 0.057 \\
34 & -0.008 & 0.317 & 0.156 & 0.105 \\
35 & 0.251 & 0.377 & 0.298 & 0.028 \\
36 & -0.002 & 0.061 & 0.026 & 0.016 \\
\hline
\end{tabular}


NET RATIO requires data for final output export share and IMPORT RATIO. The annual input-output tables for the UK published by the ONS are used to calculate the export share, which cover the period 1992-2004. So the export shares for 19881991 are also imputed with linear extrapolation. For each 2-digit final output industry each year, NET RATIO = Export Share - IMPORT RATIO. Figure 2 shows net exposure, which generally increase over time. Table 3 reports the summary statistics for each industry. Industries 30 and 32 have the highest average rate above 0.5 . Industry 20 has the lowest average rate -0.21 , which implies IMPORT RATIO is higher than the export ratio of final goods. The most stable industries are in Industry 28 with the smallest standard deviation.

To calculate NET REER, 2-digit export REER, export share, and IMPORT REER are needed. The 2-digit export REER can be easily obtained using the same data sources and same method as calculating 3-digit export REER. And we have already obtained export share and IMPORT REER from the first several measures. Figure 3 and Table 4 present the net REER indices and their statistics. The REER indices are generally stable across time and similar to the pattern of NET RATIO. Industry 32 is an exception, with the biggest standard deviation. 10 out of 22 industries have negative average net REER. Industry 21 has the lowest mean of -40 . Industries 30 and 32 have the highest mean, which is similar to those in NET RATIO.

EFFECT INDEX is obtained using the method and data sources detailed in Appendix 2. The pattern of EFFECT INDEX is shown in Figure 4. More than half of the industries are quite stable. The statistics for these indices are shown in Table 5. Industry 30 has the highest volatility. Comparing this with the 2-digit export 
Figure 3: NET REER
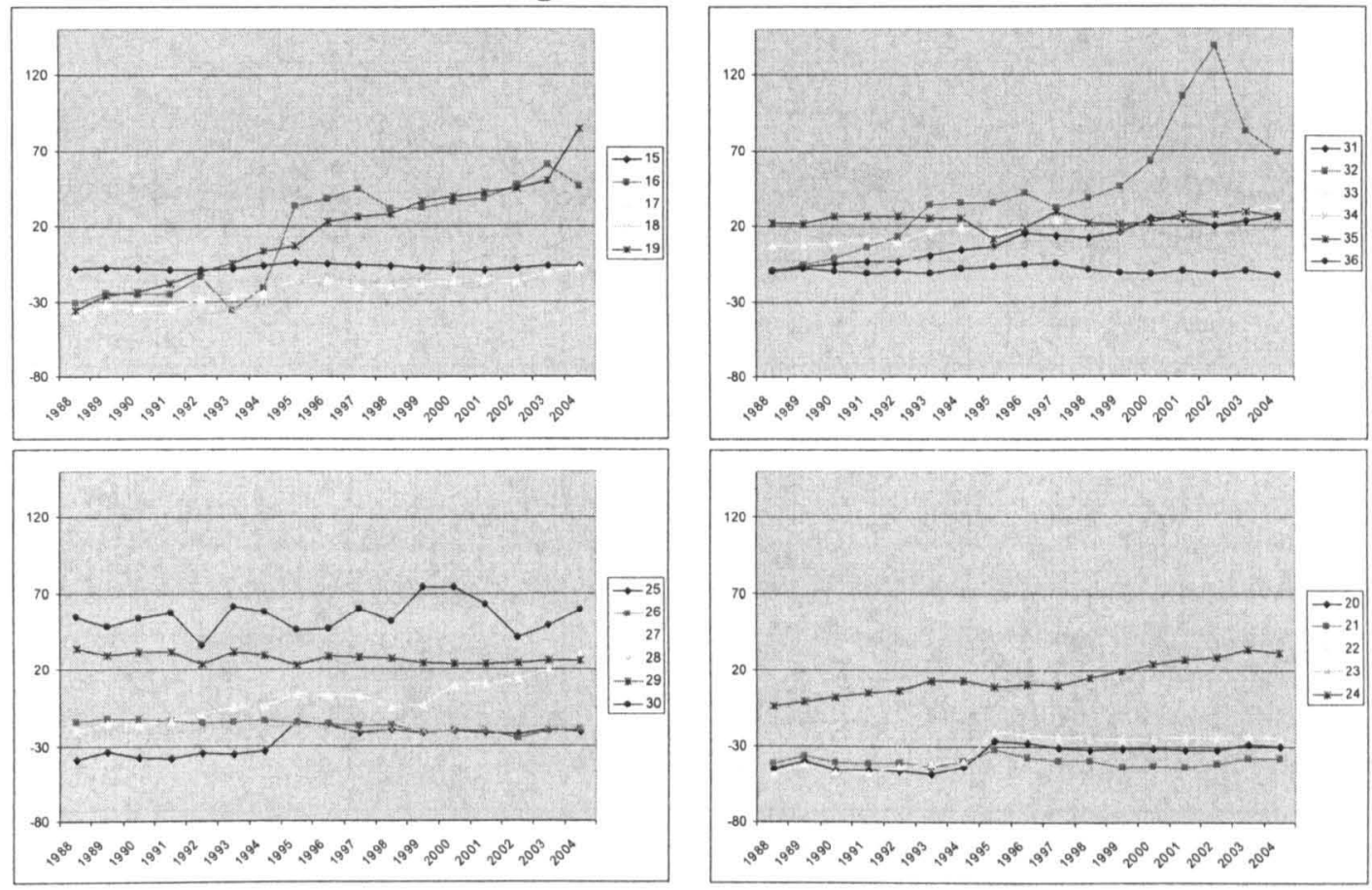

Table 4: Summary Statistics for NET REER

\begin{tabular}{crrrr}
\hline Year & Min & Max & Mean & \multicolumn{1}{c}{ SD } \\
\hline 15 & -9.292 & -3.906 & -7.187 & 1.578 \\
16 & -35.844 & 60.382 & 13.752 & 34.683 \\
17 & -38.907 & -6.752 & -22.191 & 9.094 \\
18 & -65.024 & 14.760 & -23.619 & 23.912 \\
19 & -36.303 & 84.527 & 15.886 & 32.973 \\
20 & -48.005 & -26.025 & -36.031 & 7.748 \\
21 & -43.283 & -31.844 & -39.571 & 3.037 \\
22 & -48.058 & -20.824 & -32.167 & 10.504 \\
23 & -28.060 & 25.904 & 3.913 & 18.613 \\
24 & -3.309 & 33.090 & 14.237 & 11.224 \\
25 & -38.939 & -14.946 & -26.534 & 8.348 \\
26 & -25.111 & -12.900 & -17.172 & 3.524 \\
27 & -21.158 & 29.107 & -0.194 & 13.619 \\
28 & -23.188 & -17.114 & -19.293 & 1.699 \\
29 & 23.088 & 33.360 & 27.295 & 3.272 \\
30 & 35.766 & 74.212 & 54.780 & 10.361 \\
31 & -8.757 & 26.751 & 9.468 & 12.201 \\
32 & -9.780 & 138.859 & 42.752 & 39.851 \\
33 & 7.591 & 33.492 & 18.870 & 7.845 \\
34 & -11.718 & 18.105 & 4.858 & 10.550 \\
35 & 11.475 & 29.058 & 23.854 & 4.389 \\
36 & -12.569 & -4.428 & -9.485 & 2.307 \\
\hline & & & & \\
\hline
\end{tabular}


Figure 4: EFFECT INDEX
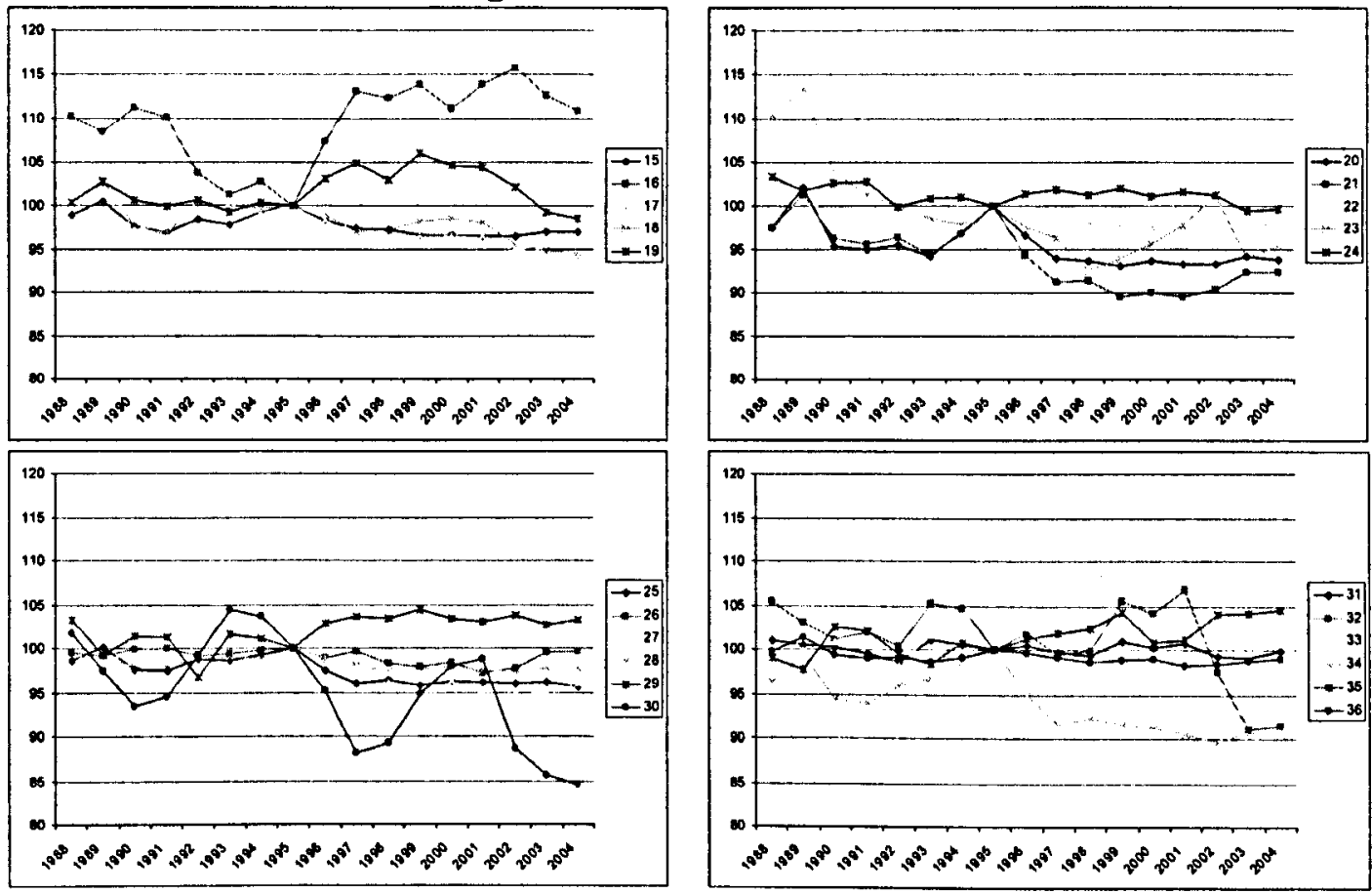

Table 5: Summary Statistics for EFFECT INDEX

\begin{tabular}{ccccc}
\hline Industry & Min & Max & Mean & SD \\
\hline 15 & 96.39 & 100.55 & 97.74 & 1.29 \\
16 & 100.00 & 115.62 & 109.30 & 4.73 \\
17 & 93.85 & 101.21 & 97.36 & 2.01 \\
18 & 94.51 & 102.86 & 98.15 & 2.17 \\
19 & 98.49 & 105.91 & 101.72 & 2.27 \\
20 & 93.11 & 102.04 & 95.42 & 2.49 \\
21 & 89.51 & 101.19 & 94.03 & 3.61 \\
22 & 97.45 & 100.46 & 98.53 & 0.92 \\
23 & 92.45 & 113.29 & 99.36 & 5.57 \\
24 & 99.35 & 103.39 & 101.27 & 1.11 \\
25 & 95.63 & 100.13 & 97.45 & 1.52 \\
26 & 97.14 & 100.00 & 99.05 & 0.86 \\
27 & 94.78 & 101.86 & 97.23 & 1.92 \\
28 & 97.27 & 100.00 & 98.19 & 0.76 \\
29 & 96.84 & 104.41 & 102.09 & 1.96 \\
30 & 84.63 & 104.46 & 95.17 & 6.12 \\
31 & 98.67 & 101.06 & 100.09 & 0.73 \\
32 & 91.14 & 106.83 & 101.18 & 4.52 \\
33 & 98.29 & 103.04 & 101.32 & 1.29 \\
34 & 89.57 & 100.00 & 94.02 & 3.34 \\
35 & 97.77 & 104.60 & 101.46 & 2.10 \\
36 & 98.25 & 101.40 & 99.21 & 0.73 \\
\hline & & & &
\end{tabular}


REER shown in Figure 5, the volatility of the value added index is much smaller than that of export REER, which may due to the offsetting effects from imported intermediate inputs. And patterns between the two are different. The correlation between the two is -0.2 as shown in Table 6 , which implies that export REER may not be a good measure of the exchange rate impact on firms when only considering the export aspect of trade.

Table 6 presents the correlation matrix between 3-digit export REER we use in our previous chapter and our two families of measures. We can see that the correlation between 3-digit export REER and measures of external orientation are quite low. Among the correlations, there are two pairs with high correlations. III REER is highly correlated with IMPORT RATIO with a correlation of 0.81 , which implies that the differences in the imported-intermediate input REER across industry mainly come from the differences in imported input ratios. The correlation between

Figure 5: 2-digit export REER index (1995=1)
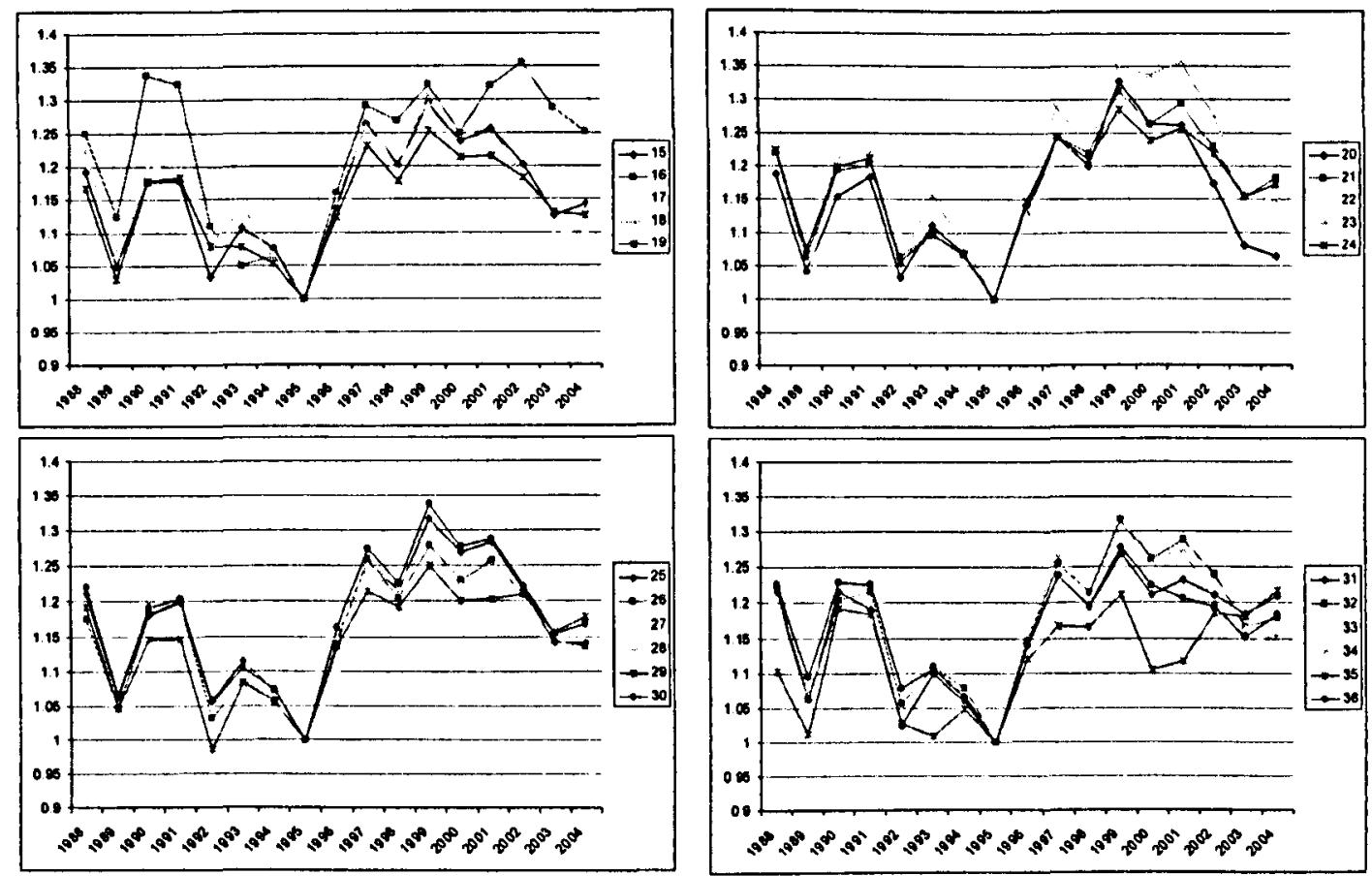
NET RATIO and NET REER is 0.96 , which is very high. So we can just regard NET RATIO as a proxy for NET REER.

Table 6: Correlation Matrix

\begin{tabular}{|l|l|l|l|l|l|l|}
\hline & $\begin{array}{l}\text { 3-digit } \\
\text { export } \\
\text { REER }\end{array}$ & $\begin{array}{l}\text { IMPORT } \\
\text { RATIO }\end{array}$ & III REER & $\begin{array}{l}\text { NET } \\
\text { RATIO }\end{array}$ & $\begin{array}{l}\text { NET } \\
\text { REER }\end{array}$ & $\begin{array}{l}\text { EFFECT } \\
\text { INDEX }\end{array}$ \\
\hline 3-digit export REER & 1.0000 & & & & & \\
\hline IMPORT RATIO & 0.0145 & 1.0000 & & & & \\
\hline III REER & 0.2261 & 0.8051 & 1.0000 & & & \\
\hline NET RATIO & 0.0428 & 0.3946 & 0.2934 & 1.0000 & & \\
\hline NET REER & 0.0484 & 0.3403 & 0.1149 & 0.9636 & 1.0000 & \\
\hline EFFECT INDEX & -0.2135 & -0.0348 & -0.1683 & 0.3050 & 0.3440 & 1.0000 \\
\hline
\end{tabular}

\section{Results after Controlling for External Orientation}

Firstly, we add the first three measures: IMPORT RATIO, III REER and NET RATIO to our Heckman selection regressions. The standard way to examine the interaction effects is to add both independent variables and the product of the two. The correlations between the interaction terms and export REER are quite low: ranging from 0.07 to 0.4 , whereas correlations between the interaction terms and measures for external orientation are very high: all above 0.95 . However, the high correlation can be avoided by mean centering the measure of external orientation. This transformation does not affect the coefficient for the interaction terms.

By adding interaction terms between export REER and the measures of external orientation, we obtain the results reported in Table 7. Adding the interaction terms and new measures has little impact on other coefficients. The main effects of export REER do not change after adding the interaction term. In Column 1 and 2, the interaction terms, in which two inward orientated IMPORT RATIO and III REER 
as measures, are only weakly significant for the former in the export intensity decision and the coefficient is positive. The results show that inward orientation has positive impact on the effects of REER on exports intensity, which is what we expected since the role of inward orientation measures should offset the negative effects of export REER on exports. So there is some evidence for our hypothesis of offsetting effects.

Table 7: Heckman Selection Model (MLE) with External Orientation

\begin{tabular}{|c|c|c|c|c|c|c|}
\hline & \multicolumn{2}{|c|}{$\begin{array}{l}\text { (1) interact with } \\
\text { IMPORT RATIO }\end{array}$} & \multicolumn{2}{|c|}{$\begin{array}{l}\text { (2) interact with } \\
\text { III REER }\end{array}$} & \multicolumn{2}{|c|}{$\begin{array}{l}\text { (3) interact with } \\
\text { NET RATIO }\end{array}$} \\
\hline & $\begin{array}{l}\text { Export } \\
\text { Dummy }\end{array}$ & $\begin{array}{l}\text { Export } \\
\text { Share }\end{array}$ & $\begin{array}{l}\text { Export } \\
\text { Dummy }\end{array}$ & $\begin{array}{l}\text { Export } \\
\text { Share }\end{array}$ & $\begin{array}{l}\text { Export } \\
\text { Dummy }\end{array}$ & $\begin{array}{l}\text { Export } \\
\text { Share }\end{array}$ \\
\hline $\begin{array}{l}\text { Lag Industry } \\
\text { REER }\end{array}$ & $\begin{array}{l}-0.001 \\
(-0.15)\end{array}$ & $\begin{array}{c}-0.0057 \\
(-2.33) \\
* *\end{array}$ & $\begin{array}{c}-0.0026 \\
(-0.35)\end{array}$ & $\begin{array}{c}-0.0055 \\
(-2.31) \\
* *\end{array}$ & $\begin{array}{r}0.0027 \\
(0.41)\end{array}$ & $\begin{array}{c}-0.0042 \\
(-2.19) \\
* *\end{array}$ \\
\hline $\begin{array}{c}\text { REER* } \\
\text { IMPORT } \\
\text { RATIO }\end{array}$ & $\begin{array}{l}0.012 \\
(1.33)\end{array}$ & $\begin{array}{c}0.006 \\
(1.78)^{*}\end{array}$ & & & & \\
\hline $\begin{array}{l}\text { IMPORT } \\
\text { RATIO }\end{array}$ & $\begin{array}{c}-2.282 \\
(-2.02)^{* *}\end{array}$ & $\begin{array}{r}-0.757 \\
(-1.67)^{*} \\
\end{array}$ & & & & \\
\hline $\begin{array}{l}\text { REER* }^{*} \\
\text { III REER }\end{array}$ & & & $\begin{array}{l}0.0001 \\
(1.50) \\
\end{array}$ & $\begin{array}{c}0.00003 \\
(1.46)\end{array}$ & & \\
\hline III REER & & & $\begin{array}{c}-0.016 \\
(-1.77)^{*}\end{array}$ & $\begin{array}{l}-0.0035 \\
(-1.27)\end{array}$ & & \\
\hline $\begin{array}{c}\text { REER* } \\
\text { NET RATIO }\end{array}$ & & & & & $\begin{array}{l}-0.005 \\
(-1.11)\end{array}$ & $\begin{array}{c}0.0003 \\
(2.26)^{* *}\end{array}$ \\
\hline NET RATIO & & & & & $\begin{array}{l}0.789 \\
(1.31)\end{array}$ & $\begin{array}{c}-0.337 \\
(-2.21)^{* *}\end{array}$ \\
\hline $\begin{array}{c}\text { Lambda } \\
\text { (standard } \\
\text { error) } \\
\end{array}$ & \multicolumn{2}{|c|}{$\begin{array}{c}-0.0339 \\
(0.0057)^{* * *}\end{array}$} & \multicolumn{2}{|c|}{$\begin{array}{c}-0.0341 \\
(0.0057)^{* * *}\end{array}$} & \multicolumn{2}{|c|}{$\begin{array}{c}-0.0340 \\
(0.0057)^{* * *}\end{array}$} \\
\hline $\begin{array}{c}\text { Rho } \\
\text { (standard } \\
\text { error) }\end{array}$ & \multicolumn{2}{|c|}{$\begin{array}{c}-0.1325 \\
(0.021)^{* * *}\end{array}$} & \multicolumn{2}{|c|}{$\begin{array}{c}-0.1330 \\
(0.021)^{* * *}\end{array}$} & \multicolumn{2}{|c|}{$\begin{array}{c}-0.1330 \\
(0.021)^{* * *}\end{array}$} \\
\hline
\end{tabular}

Observations: 44,251 Firms: 5, 876

(i) $\mathrm{Z}$ statistics in parentheses, robust standard errors adjusted for 83 clusters in 3-digit industries.

(ii) *significant at $10 \%$; ${ }^{* *}$ significant at $5 \%$; ${ }^{* *}$ significant at $1 \%$

(iii) $\rho$ is the estimated correlation between the error terms of the two equations; if it is different from zero it suggests that the two equations are related and that the selection model is appropriate; $\lambda$ is the estimated coefficients of the inverse Mills ratio; if it is different from zero it suggests that there is sample selection. 
In Column 3, the interaction term, in which NET RATIO is the external orientation measure, is positive and significant in export share decision. this may seem counter-intuitive as we expect its reinforce effect on export REER. However, it is not surprising, since the measure may be capturing other factors embodied in the trade index, which may lead to unexpected results for external orientation.

Then, we include NET REER and EFFECT INDEX in our regression. Since the index has already taken export related REER into account, we replace export REER with the two measures to examine whether changes in net external orientation and value added due to exchange rate movement could affect the export decision of firms. Column 1 of Table 8 reports the results for NET REER. The coefficients are always insignificant. The results of Column 2 in table 8 show that EFFECT INDEX does not have any significant effect on export decisions of firms. There is a possibility that value added changes will induce changes in the wages of their employees. That is: if firms get more value added, they may increase wages; whereas if their value added decreases, the wage would shrink accordingly. So there may be a correlation between EFFECT INDEX and the independent variable, real wage, in our regression. We therefor drop the variable "wage" in the regression. Column 3 reports the results, which show that dropping wage does not change the results for EFFECT INDEX. So there is no evidence for correlation between the two. The insignificance of the coefficients for the NET RATIO and EFFECT INDEX supports our prediction that the effects of net and effective indices on export behavior may be weaker than those of export REER due to offsetting effects between inward and outward orientation. 
Table 8: Heckman Selection Model (MLE) with NET REER and EFFECT INDEX

\begin{tabular}{|c|c|c|c|c|c|c|}
\hline & \multicolumn{2}{|c|}{ (1) NET REER } & \multicolumn{2}{|c|}{ (2) EFFECT INDEX } & \multicolumn{2}{|c|}{$\begin{array}{l}\text { (3) EFFECT INDEX } \\
\text { without wage }\end{array}$} \\
\hline & $\begin{array}{l}\text { Export } \\
\text { Dummy }\end{array}$ & $\begin{array}{l}\text { Export } \\
\text { Share }\end{array}$ & $\begin{array}{l}\text { Export } \\
\text { Dummy }\end{array}$ & $\begin{array}{l}\text { Export } \\
\text { Share }\end{array}$ & $\begin{array}{l}\text { Export } \\
\text { Dummy }\end{array}$ & $\begin{array}{l}\text { Export } \\
\text { Share }\end{array}$ \\
\hline $\begin{array}{l}\text { Lag Export } \\
\text { dummy }\end{array}$ & $\begin{array}{c}3.04 \\
(39.80) \\
* * *\end{array}$ & & $\begin{array}{c}3.04 \\
(39.93) \\
* * *\end{array}$ & & $\begin{array}{c}3.04 \\
(40.12) \\
* * *\end{array}$ & \\
\hline $\begin{array}{l}\text { Lag NET } \\
\text { REER }\end{array}$ & $\begin{array}{l}0.0006 \\
(0.39)\end{array}$ & $\begin{array}{c}0.00023 \\
(0.58)\end{array}$ & & & & \\
\hline $\begin{array}{c}\text { Lag } \\
\text { EFFECT } \\
\text { INDEX } \\
\end{array}$ & & & $\begin{array}{r}-0.0079 \\
(-1.61)\end{array}$ & $\begin{array}{r}0.0002 \\
(0.13)\end{array}$ & $\begin{array}{r}-0.0078 \\
(-1.61)\end{array}$ & $\begin{array}{r}0.0002 \\
(0.13)\end{array}$ \\
\hline $\begin{array}{l}\text { Lag log of } \\
\text { employment }\end{array}$ & $\begin{array}{c}0.0436 \\
(2.31)^{* *}\end{array}$ & $\begin{array}{l}0.0019 \\
(0.39)\end{array}$ & $\begin{array}{c}0.0432 \\
(2.30)^{* *}\end{array}$ & $\begin{array}{r}0.0029 \\
(0.60)\end{array}$ & $\begin{array}{c}0.0434 \\
(2.31)^{* *}\end{array}$ & $\begin{array}{r}0.0029 \\
(0.60)\end{array}$ \\
\hline $\begin{array}{l}\text { Lag log of } \\
\text { wage }\end{array}$ & $\begin{array}{l}0.0357 \\
(0.72)\end{array}$ & $\begin{array}{c}0.093 \\
(3.12) \\
* * * \\
\end{array}$ & $\begin{array}{l}0.0557 \\
(1.15)\end{array}$ & $\begin{array}{c}0.0914 \\
(3.09) \\
* * *\end{array}$ & & \\
\hline $\begin{array}{c}\text { Lag log of } \\
\text { labor } \\
\text { productivity }\end{array}$ & $\begin{array}{r}0.0376 \\
(1.21)\end{array}$ & $\begin{array}{r}-0.0106 \\
(-1.14)\end{array}$ & $\begin{array}{r}0.0307 \\
(1.00)\end{array}$ & $\begin{array}{l}-0.0101 \\
(-1.59)\end{array}$ & $\begin{array}{l}0.048 \\
(1.37)\end{array}$ & $\begin{array}{c}0.0154 \\
(1.79) \\
*\end{array}$ \\
\hline $\begin{array}{c}\text { Lag log of } \\
\text { age }\end{array}$ & $\begin{array}{r}-0.0245 \\
(-1.57)\end{array}$ & $\begin{array}{c}-0.0095 \\
(-2.50) \\
* *\end{array}$ & $\begin{array}{r}-0.0247 \\
(-1.59)\end{array}$ & $\begin{array}{c}-0.0096 \\
(-2.25) \\
* *\end{array}$ & $\begin{array}{l}-0.0245 \\
(-1.58)\end{array}$ & $\begin{array}{c}-0.0086 \\
(-2.25) \\
* *\end{array}$ \\
\hline $\begin{array}{l}\text { Foreign } \\
\text { owner } \\
\text { dummy }\end{array}$ & $\begin{array}{c}0.1315 \\
(4.25) \\
* * *\end{array}$ & $\begin{array}{c}0.0584 \\
(6.82) \\
* * *\end{array}$ & $\begin{array}{c}0.1317 \\
(4.23) \\
* * *\end{array}$ & $\begin{array}{c}0.06 \\
(7.40) \\
* * *\end{array}$ & $\begin{array}{c}0.1333 \\
(4.36) \\
* * *\end{array}$ & $\begin{array}{c}0.06 \\
(7.63) \\
* * *\end{array}$ \\
\hline $\begin{array}{c}\text { Lambda } \\
\text { (standard } \\
\text { error) } \\
\end{array}$ & $\begin{array}{c}-0.0341 \\
(0.0057)^{* *} \\
*\end{array}$ & & $\begin{array}{c}-0.0340 \\
(0.0057) \\
* * *\end{array}$ & & $\begin{array}{c}-0.0332 \\
(0.0057) \\
* * *\end{array}$ & \\
\hline $\begin{array}{c}\text { Rho } \\
\text { (standard } \\
\text { error) } \\
\end{array}$ & $\begin{array}{c}-0.1331 \\
(0.021)^{* * *}\end{array}$ & & $\begin{array}{c}-0.1326 \\
(0.0210) \\
* * *\end{array}$ & & $\begin{array}{c}-0.129 \\
(0.0214) \\
* * *\end{array}$ & \\
\hline
\end{tabular}

Notes: See notes in Table 7.

After controlling for external orientation, we look at the industry heterogeneity in the effects of exchange rate changes to see whether there are any changes. We split the sample into twenty-two 2-digit level industries and use EFFECT INDEX instead of the export REER used in Chapter 2 to run the regressions. We use EFFECT INDEX as the exchange rate measure rather than NET REER, as the former examines changes in value added due to exchange rate movement. Table 9 
reports the results for each 2-digit industry and Table 10 shows the summary of the effects reported in Table 9 across 2-digit industries, in which the industries are split into 5 categories according to different effects in export entry and export intensity decisions.

Table 9: Industry Heterogeneity Using EFFECT INDEX as REER Measure

\begin{tabular}{|c|c|c|}
\hline $\begin{array}{c}\text { Industry } \\
\text { code }\end{array}$ & $\begin{array}{c}\text { Export } \\
\text { dummy }\end{array}$ & $\begin{array}{c}\text { Export } \\
\text { share }\end{array}$ \\
\hline 15 & + & + \\
\hline 17 & + & $+^{*}$ \\
\hline 18 & $+* * *$ & $+* * *$ \\
\hline 20 & - & + \\
\hline 22 & + & + \\
\hline 24 & - & - \\
\hline 26 & $+^{*}$ & + \\
\hline 27 & - & $-* * *$ \\
\hline 28 & + & - \\
\hline 29 & + & + \\
\hline 31 & $+* *$ & $+* * *$ \\
\hline 32 & $-* *$ & - \\
\hline 33 & $+* * *$ & + \\
\hline 34 & $+*$ & - \\
\hline 35 & n.a. & n.a. \\
\hline 36 & + & $-* * *$ \\
\hline
\end{tabular}

* indicates significant at $10 \% ;{ }^{* *}$ indicates significant at $5 \% ;{ }^{* * *}$ indicates significant at $1 \%$

Now we can compare Table 10 with Table 17 in Chapter 2. It is obvious that the effects become quite different for most of industries after controlling for external orientation. Only seven industries (Industries 15, 20, 24, 27, 29, 33 and 34) have similar effects as before. Two (Industries 32 and 36) change from positive effects to negative effects; two industries (Industries 22 and 26) change from negative effects to insignificant effects, four (Industries 17, 18, 26, and 31) from insignificant to positive. When we look at Table 1 showing IMPORT RATIO for each 2-digit manufacturing industry, the ratios for Industries 17,18 and 32 are among the top with largest imported intermediate inputs relative to total intermediate inputs. (Unfortunately, results for some of the industries with large 
import ratios are unavailable due to the small number of observations) So superficially we have a plausible explanation for the changes after controlling of external orientation: it may be imported intermediate inputs that play a role in the effects of exchange rate on exports. There are other factors affecting the effects, as there is still industry heterogeneity after controlling for external orientation.

Table 10: Summary of Industry Heterogeneity after Controlling for External Orientation (EFFECT INDEX as REER Measure)

\begin{tabular}{|c|c|}
\hline $\begin{array}{l}\text { Different } \\
\text { effect type }\end{array}$ & Industries \\
\hline $\begin{array}{l}\text { export entry } \\
\text { (negative), } \\
\text { export share } \\
\text { (insignificant } \\
\text { or negative) }\end{array}$ & $\begin{array}{l}32 \text { Manufacture of Radio, Television and Communication } \\
\text { Equipment and Apparatus }\end{array}$ \\
\hline $\begin{array}{l}\text { export entry } \\
\text { (insignificant) } \\
\text { export share } \\
\text { (negative) } \\
\end{array}$ & $\begin{array}{l}27 \text { Manufacture of Basic Metals } \\
36 \text { Manufacture of Furniture; Manufacturing Not Elsewhere } \\
\text { Classified }\end{array}$ \\
\hline $\begin{array}{l}\text { Insignificant in } \\
\text { both decisions }\end{array}$ & $\begin{array}{l}15 \text { Manufacture of Food Products and Beverages } \\
20 \text { Manufacture of Wood and Products of Wood and Cork, } \\
\text { Except Furniture; Manufacture of Articles of Straw and } \\
\text { Plaiting Materials } \\
22 \text { Publishing, Printing and Reproduction of Recorded Media } \\
24 \text { Manufacture of Chemicals and Chemical Products } \\
28 \text { Manufacture of Fabricated Metal Products, Except } \\
\text { Machinery and Equipment } \\
29 \text { Manufacture of Machinery and Equipment Not Elsewhere } \\
\text { Classified }\end{array}$ \\
\hline $\begin{array}{l}\text { export entry } \\
\text { (insignificant) } \\
\text { export share } \\
\text { (positive) }\end{array}$ & 17 Manufacture of Textiles \\
\hline $\begin{array}{l}\text { export entry } \\
\text { (positive) } \\
\text { export share } \\
\text { (insignificant } \\
\text { or positive) }\end{array}$ & $\begin{array}{l}18 \text { Manufacture of Wearing Apparel; Dressing and Dyeing of } \\
\text { Fur } \\
26 \text { Manufacture of Other Non-metallic Mineral Products } \\
31 \text { Manufacture of Electrical Machinery and Apparatus Not } \\
\text { Elsewhere Classified } \\
33 \text { Manufacture of Medical, Precision and Optical Instruments, } \\
\text { Watches and Clocks } \\
34 \text { Manufacture of Motor Vehicles, Trailers and Semi-trailers }\end{array}$ \\
\hline
\end{tabular}




\section{Conclusions}

In this chapter, we controlled for external orientation to investigate whether taking the level of external orientation and imported intermediate inputs into consideration would affect our results for the relationship between exchange rate movements and export decisions of firms, and thus expected to partly explain industry heterogeneity in the effects of exchange rate changes on exports. We used several new measures of inward orientation and external orientation to examine the issue. We find some evidence for offsetting effects between inward and outward orientation. Further investigation in some other aspects, for example, the market structure, may suggest other ways to explain industry heterogeneity. We will investigate this issue further in the next chapter. 


\section{Appendix 1: Calculating Imported Intermediate Input REER (III REER)}

To calculate III REER, we have to firstly calculate annual import weighted REER for each 2-digit tradable product industry ${ }^{5}$ (i.e. each intermediate inputs industry, in which the goods will be used as intermediate inputs to produce outputs in final goods industries) using the following equation as in export REER:

$$
i m R E E R=\prod_{i}\left[\left(e_{i} / e\right)\left(p / p_{i}\right)\right]^{w_{i}}
$$

Where $e_{i}$ : Exchange rate of currency $i$ against Special Drawing Rights (annual average)

(Units of Currency $i$ per SDR in index form, 1995 as the base year)

e: Exchange rate of GBP against Special Drawing Rights (annual average)

(Units of GBP per SDR in index form, 1995 as the base year)

p: Price index of UK (using inflation index as a proxy, 1995 as the base year)

$\mathrm{p}_{i}$ : Price index of country $i$ (using inflation index as a proxy, 1995 as the base year)

$w_{i}$ : the import value from import origin country $i$ relative to the total import value from abroad in each 2-digit industry

The imREER index is the import REER index for each intermediate input industry. Trade data for import weight is from two sources: OECD bilateral trade commodity data and trade commodity data from www.uktradeinfo.com. Here we express the exchange rate in terms of foreign currency value of a unit of the domestic currency. An upward movement therefore represents appreciation of sterling and imported input prices decrease accordingly, and a downward movement represents depreciation and thus a rise in imported input prices.

After obtaining the import REER for each intermediate tradable input industry, we multiply it by the imported intermediate input ratio, since only imported inputs

\footnotetext{
${ }^{5}$ In the exchange rate pass-through literature, the exchange rate pass-through is high for tradable goods, but low for nontradable goods. For simplicity, here we only consider the exchange rate passthrough in tradable goods.
} 
incur exchange rate shocks whereas domestic intermediate inputs are not affected by exchange changes. Then the annual imported intermediate input REER for each 2-digit manufacturing output industry is obtained by:

$$
\text { Imported-intermediate-input } R E E R_{i}=\sum_{j} i m R E E R_{j}{ }^{*} M_{i j}
$$

Where $i$ : 2-digit output industry

$j$ : intermediate input industry (tradable products) to produce final products in $i$

$M_{i j}$ : imported intermediate input share of industry $j$ in producing products of industry $i$, calculated as imported intermediate inputs value for industry $j$ relative to total input value (tradable and nontradable) for all intermediate input industries to produce final products in industry $i$.

imREER $R_{j}$ import REER index for intermediate inputs industry $j$, calculated from Equation (1)

Here we have to assume that the imported inputs are evenly distributed across intermediate input use and final use and input-output relationships are identical across firms in a given industry.

Data available for imported intermediate inputs ratio is from UK input-output tables in 1990 and 1995(data for 1998 cannot be used here due to the aggregation in some industries). Since there are only two annual data series, we choose not to use linear regression to fill up the missing value. So we are assuming fixed imported ratio, and use data for 1990 to cover the period 1988-1994 and data for 1995 to period $1995-2004$. 


\section{Appendix 2: Calculating Effective External Orientation Index (EFFECT}

\section{INDEX)}

The NET REER measure in Family 2 simply takes the input and output of products at the same scale, which is not realistic. The value added part for each product is what influences the decision of firms. To be precise and accurate, we need to see to what extent exchange rates affect value added of a firm and thus influence firm's export decisions. So we calculate an effective external orientation index. The effective value added index borrows the method for computing effective protection in the trade policy literature. The main idea is to generate an index to represent the changes in value added due to exchange rate changes on both imported intermediate inputs and exported outputs within an industry. To elaborate the concept formally, industry $j$ purchases inputs of $i$, combines these inputs to add value and produces output of $\mathrm{j}$. The percentage changes in value added can be defined as:

$$
T_{j}=\frac{v_{j}^{1}-v_{j}^{0}}{v_{j}^{0}}=\frac{v_{j}^{1}}{v_{j}^{0}}-1
$$

Where $v_{j}^{0}$ is the value added to the final product $\mathrm{j}$ with no international exposure to trade and $v_{j}^{1}$ is the value added to the final product $\mathrm{j}$ with external exposure to trade and incur exchange rate movement impacts ${ }^{6}$. We define $v_{j}^{0}$ and $v_{j}^{1}$ as:

$$
v_{j}^{0}=p_{j}\left\{1-\sum_{i} a_{i j}\right\}
$$

\footnotetext{
${ }^{6}$ For effective protection, value added of product $\mathrm{j}$ is $v_{j}^{r}=p_{j}\left\{\left(1+r_{j}\right)-\sum_{i} a_{i j}\left(1+r_{i}\right)\right\}$, where $r_{i}$ and $r_{j}$ are tariff rates for input $i$ and final product $j$. the counter part in the case of external exposure to exchange rate changes becomes Equation (3).
} 


$$
v_{j}^{1}=\left[(1-\beta) p_{j}+\beta\left(1+t_{j}\right) p_{j}^{*}\right]\left\{1-\sum_{i} a_{i j}\left[\lambda_{i}\left(1+t_{i}\right)+\left(1-\lambda_{i}\right)\right]\right\}
$$

where $p_{j}$ is the domestic price of the final product $j, p_{j}^{*}$ is the world price, $\beta$ is export share for final product $\mathrm{j}, \mathrm{t}_{\mathrm{j}}$ is the percentage change of exchange rate for exporting product $\mathrm{j}, \mathrm{t}_{\mathrm{i}}$ is the percentage change of exchange rate for importing input $i, a_{i j}$ is the share of final value of $j$ accounted for by input $i . \lambda_{i}$ is the imported intermediate inputs (tradable) relative to the total intermediate inputs of $i$. We use similar assumptions as in computing effective protection and further assume $p_{j}=$ $\mathrm{p}_{\mathrm{j}}^{*}$. Substitute (3) and (4) into (2), we have

$$
\begin{aligned}
\mathrm{T}_{j} & =\frac{\left[(1-\beta) p_{j}+\beta\left(1+t_{j}\right) p_{j}^{*}\right] *\left[1-\sum_{i} a_{i j}\left[\lambda_{i}\left(1+t_{i}\right)+\left(1-\lambda_{i}\right)\right]\right]-p_{j}\left(1-\sum_{i} a_{i j}\right)}{p_{j}\left(1-\sum_{i} a_{i j}\right)} \\
& =\frac{\left(1+\beta t_{j}\right)\left[1-\sum_{i} a_{i j}\left(\lambda_{i} t_{i}+1\right)\right]-\left(1-\sum_{i} a_{i j}\right)}{1-\sum_{i} a_{i j}} \\
& =\frac{\beta t_{j}-\left(1+\beta t_{j}\right) \sum_{i} a_{i j}\left(\lambda_{i} t_{i}+1\right)+\sum_{i} a_{i j}}{1-\sum_{i} a_{i j}} \\
& =\frac{\beta t_{j}-\left(1+\beta t_{j}\right)\left(\sum_{i} a_{i j}+\sum_{i} a_{i j} \lambda_{i} t_{i}\right)+\sum_{i} a_{i j}}{1-\sum_{i} a_{i j}} \\
& =\beta t_{j}-\frac{\left(1+\beta t_{j}\right) \sum_{i} a_{i j} \lambda_{i} t_{i}}{1-\sum_{i} a_{i j}}
\end{aligned}
$$

So the percentage changes for value added are affected by $\beta, t_{j}, t_{i}, a_{i j}$ and $\lambda_{i}$. This value added change has quite strict assumptions, and potential conflicts as, for example, between the implicit dynamic assumption and static framework. 
However, we still regard it as a feasible proxy for the value added changes due to exchange rate movements. Campa and Goldberg (1995) and Campa and Goldberg (1999) use a different way to examine the exposure to exchange rate movements. Many assumptions can be relaxed in their theoretical equation. But it would be quite complex to measure it according to their equations, so they end up with the net exposure rate as the simple measure.

Data for $\beta, a_{i j}$ and $\lambda_{i}$ are obtained from IO tables. For some missing values in certain years, we use linear extrapolation. For each year, Data for $t_{j}$ and $t_{i}$ is obtained by computing respectively the percentage changes of export REER and imREER which we have obtained from previous measures in 2-digit industry level. After obtaining percentage change $\mathrm{Tj}$, we then transfer percentage change $\mathrm{Tj}$ to the value added index (set $1995=100$ ), i.e. effective external orientation index (EFFECT INDEX). 


\section{Chapter 4}

\section{Explaining Industry Heterogeneity: the Role of Pricing to Markets}

\section{Introduction}

In Chapter 3, we explained cross-industry differences in the effect of the level of the exchange rate on firms' exports by taking the cost impact of the exchange rate into consideration. However, this may not be the only factor driving heterogeneity. This chapter aims to explore another possible driver by drawing upon the literature of exchange rate pass-through. Within this literature, there are a number of theoretical and empirical papers (such as Krugman 1987, Dornbusch 1987, Faruqee 1995, Clark and Faruqee 1997, and Feenstra and Kendall 1997) that attempt to explain the apparently low level of exchange rate pass-through by firms' discriminatory pricing behavior, termed as pricing to market (PTM hereafter). Marston (1990) describes PTM as "pricing policies designed to keep export price competitive despite changes in exchange rates". Highly incomplete exchange rate pass-through due to firms' PTM behaviour stabilizes export prices in terms of foreign currency in destination markets, and thus cushions the impact of exchange rate changes on exports. PTM, therefore, provides a potentially important explanation for differences in exchange rate pass-through across industries.

PTM involves imperfect competition. As stated by Krugman (1987): "the intellectual interest of PTM is that it offers evidence on the role of market structure in international trade". Thus PTM provides us with a way to connect market structure, exchange rate changes and export behavior. We expect that exports in sectors with a high degree of PTM and low degree of exchange rate pass-through 
are less negatively affected by exchange rate movements in terms of local currencies, as PTM behaviour stabilizes the demand from export markets.

To empirically examine whether PTM plays a role, we need to identify potential factors inducing incomplete exchange rate pass-through and the determinants of its magnitude. Although theoretical models are based on a wide-ranging set of assumptions, they can provide us with some of the main factors and determinants of PTM. Moreover, we draw upon existing empirical papers to suggest possible measures to capture the important features of PTM. So the theoretical models are summarized in Section 2, and related measures empirical papers used are shown in Section 3. In Section 4, we present our measures. These are then used to test whether PTM affects the role of exchange rate changes on export decisions. Methods to account for interaction effects are discussed in Section 5. Our empirical results are reported in Section 6, and Section 7 concludes.

\section{Theoretical Background: Determinants of PTM}

In an early paper by Dornbusch (1987), the monopolistic firm's pricing power is a function of demand elasticity, which in turn depends on substitutability among varieties within an industry. The relative price adjustment to exchange rate movements depends on product differentiation, the relative number of domestic and foreign firms, product substitutability and market structure. Exchange rate pass-through should be lower in industries in which firms have larger market power with high markups and in countries with large share of domestic firms. When markets are segmented and elasticities of demand are not constant, a monopolistic firm's optimal pricing behavior in response to exchange rate changes leads to price 
discrimination by market destination. Such pricing behavior was described as PTM by Krugman (1987), in which some suggestive theoretical models are presented to explain the phenomenon. Krugman (1987) argues that a full explanation of PTM should come from dynamic models of imperfect competition. Market shares, elasticity of demand, together with supply dynamics (such as costs of adjusting to international markets and distributional infrastructure) and demand dynamics (such as investment in reputation) can be the key elements to understand PTM. In other early papers, such as Feenstra (1989), Marston (1990) and Knetter (1995), PTM is a function of the convexity of the demand curve in the export market. Changes in marginal costs due to changes in output level also influence PTM in Marston (1990). Kasa (1992) builds a dynamic model to show that adjustment costs can generate short-run PTM. And as Knetter (1993) points out, adding competitors will increase the likelihood of observing PTM.

Of interest to this chapter are those models that attempt to measure PMT using measures of international trade. Faruqee (1995) develops a dynamic general equilibrium model of market segmentation and PTM to investigate the consequences of PTM for exchange rate pass-through, and finds that across different patterns of trade (inter-industry trade and intra-industry trade), significant variation exists in the degree of pass-through. For inter-industry trade, as the elasticity of substitution among varieties increases, the degree of PTM decreases. For intra-industry trade, the degree of PTM increases with this elasticity. In an intermediate structure between the two, the degree of PTM would be increasing with the elasticity of substitution. So PTM depends on the degree of intra-industry trade and elasticity of substation among varieties. Clark and Faruqee (1997) extend 
the two-country market segmentation and PTM model of Faruqee (1995) to analyze the consequences of PTM for the effects of exchange rate changes on the levels and variance of export prices and quantities, and finds the effects are considerably dampened. They also explore the effects of exchange rate uncertainty and finds that unpredictable volatility of exchange rates has comparatively small effects in raising the level of export prices and reducing the volume of international trade.

Yang (1998)'s theoretical model shows that PTM in the domestic market depends on the future and existing market share, the product differentiation, and the marginal costs. Recently, Atkeson and Burstein (2008) build a model with two key elements: imperfect competition with variable markups and international trade costs $^{1}$. The model is then used to assess which features of market structure are important in generating PTM. They use two measures of market structure: export participation, and the extent of within-sector cost dispersion across firms. The quantitatively most important feature of market structure that generates PTM in the paper is the within-sector cost dispersion. In their model, it is only the large firms that experience PTM in the direction suggested by the aggregate data.

Although distribution costs have not formally been included in PTM theoretical models, ${ }^{2}$ most recent papers find that they are important. Choudhri, Faruqee and Hakura (2005) examine the relative performance of different macroeconomic models in explaining exchange rate pass-through and find that the introduction of distribution costs improves the fit of the models substantially from the evidence for

\footnotetext{
'In that paper, international trade costs are export entry sunk costs and iceberg type marginal costs ${ }^{2}$ In a model of producer currency pricing (the opposite of PTM or local currency pricing), Corsetti and Dedola (2002) show that local distribution costs influence the price elasticity of demand which is then sensitive to the exchange rate.
} 
non-US G7 countries. They argue that PTM models should be extended to include distribution costs. Using data for 21 OECD countries, Campa and Goldberg (2006) find that distribution costs take up " 32 to 50 percent of total cost of goods across OECD countries", and they are crucial in explaining exchange rate pass-through. In support of this, Goldberg and Hellerstein (2007) empirically identify the sources of local-currency price stability using micro data from the beer market in the US and find that $54.1 \%$ of the incomplete exchange rate pass-through is due to local nontraded costs (destination market specific costs for non-traded services); $33.7 \%$ to markup adjustment; and $12.2 \%$ to fixed costs of repricing (price adjustment costs) ${ }^{3}$. Distribution costs which may be embodied in local non-traded costs and costs of repricing, are therefore an important source of incomplete transmission of exchange rate variation.

Theoretically, there are many possible explanations of PTM and incomplete exchange rate pass-through. Main factors include: market shares, convexity of demand curve, adjustment costs, inter-industry versus intra-industry trade patterns, international trade costs, and distribution costs. As we have seen, each paper highlights some of the factors under various assumptions and frameworks. These models motivate empirical work in this area.

\section{Empirical Literature: Measures of the Determinants}

Empirical papers have employed various measures to operationalize the factors suggested by theories. Most papers focus on PTM behavior within the context of a

\footnotetext{
${ }^{3}$ In that paper, the fixed costs of repricing include "menu-costs", the more substantive costs to figure out the new optimal price, the additional costs of advertising and more generally communicating the price change to the consumers, and so on.
} 
nation's domestic market. However, as our focus is the export behavior of UK firms under exchange rate changes, i.e. their behavior in international markets, the measurement becomes more complicated. We first summarize the stylized facts reported in the empirical literature, then turn to measures of the determinants used in empirical papers, which in turn provide a basis for the measures we employ.

Knetter (1993) makes international comparisons of PTM using U.S, U.K., German, and Japanese industry-level data. The main finding is that industry specific factors are the critical dimension in explaining PTM rather than export destinations or source countries. The evidence shows that German and Japanese exporters practice PTM, while U.S exporters show little evidence of PTM. To explain this difference, they suggest a role of FDI from the US to other destination markets. Rangan and Lawrence (1993) have used a similar argument to explain that the lack of PTM in US export industries is related to FDI. Using a somewhat different approach, Feenstra and Kendall (1997) find PTM contributing substantially to the postBretton Woods PPP deviations among G5 countries. Interestingly, although evidence shows that PTM is prevalent in industries with heterogeneous products, there is evidence of PTM in some industries with homogenous products, such as chemical products (Knetter, 1993) and linerboard paper (Goldberg and Knetter, 1997).

The empirical literature on PTM is well summarized in Goldberg and Knetter (1997), who state: "while the PTM literature has delivered convincing evidence of price discrimination, there have been fewer attempts to identify the determinants of the magnitude of PTM across industries." This occurs because of difficulties in 
identifying and measuring the determinants across industries. PTM behavior is the outcome of market power. Market power and market structure are aspects of industrial organization and they are always analyzed in a country's domestic market. At the same time, PTM is a phenomenon in international markets. It becomes more complicated to measure an exporter's market power in international or foreign markets. Goldberg and Knetter (1999) propose a novel method to measure the intensity of competition in export markets with extensive product differentiations. They calculate the elasticity of a firm's residual demand as a proxy for the intensity of competition exporters face in a particular export market. Exchange rate fluctuations are then used as a cost shifter in identifying the residual demand elasticity in various export destination markets. Unfortunately, since data for each market are needed to compute this elasticity, this method cannot be applied in this study due to the unavailability of data and complexity of calculation.

In a most recent paper, using yearly data for five main euro area countries over the period of 1990-1999, Bugamelli and Tedeschi (2008) find that exchange rate passthrough is "highly incomplete for sales by oligopolistic industries into advanced economics", and is "almost complete in emerging and developing economies". The paper tries to explain exchange rate pass-through heterogeneity in terms of market structure. Manufacturing industries are divided into four categories as in Paviit (1984): traditional sectors, industries dominated by specialized suppliers, scaleintensive industries, and science-based industries. And they regard industries (called oligopolistic industries in the paper) in latter categories have more market power than those in the former categories due to economies of scales and innovation. And the evidence shows that PTM is more likely to happen in those 
oligopolistic industries. They also consider the role of destination markets characteristics. Contrary to Knetter (1993)'s assertion that characteristics of export destination is unimportant, they find low level of exchange rate pass-through in intra-industry-trade between advanced countries and almost complete pass-through in inter-industry trade between developed and developing countries. They argue that differences across destination markets have much larger impact on the level of exchange rate pass-through than those across industries.

We summarize some stylized features and measures of determinants of PTM in the existing PTM and exchange rate pass-through literature as follows: markups (such as price-cost margin), trade patterns (intra-industry trade vs. inter-industry trade), R\&D intensity, types of scale of economy, market concentration (such as Herfindahl indices), international trade costs and so on. According to the theoretical models, the elasticity of substitution among varieties is one of the determinants of PTM. The issue of measuring this has received relatively little attention due to its inherent difficulty. ${ }^{4}$

\section{Measuring the Determinants of PTM}

\subsection{Intra-industry trade index}

According to the theoretical literature, one of the key determinants of PTM is trade patterns: specifically the balance between inter-industry and intra-industry trade. The trade pattern determines the extent to which firms face international

\footnotetext{
4 Since measures of elasticities of substitution among varieties are applied in other areas of economics, these literatures (such as Anderson, 1979; Romer, 1994; Hummels, 1999; Feenstra 1994; and Broda and Weinstein, 2006) provide possible alternative measures. Their methods are complicated and require good data. However, they provide us with a broader horizon to understand and measure the elasticity.
} 
competition in domestic and export markets, and affects PTM. As pointed out in Faruqee (1995), trade patterns and patterns of industry specialization largely determine the degree of strategic complementarity or price linkage between producers in different countries. Under inter-industry trade, countries specialize and trade according to comparative advantage. This pattern of trade, usually trade between north and south, is the traditional $\mathrm{H}-\mathrm{O}$ view of trade, which is due to different factor endowments. The absence of local competitors in each export destination country may limit producers' concerns about international relative prices. Under intra-industry trade, often observed between OECD countries, countries with identical factor endowments gain from scale economies and product differentiation. Domestic and foreign products are close substitutes, which implies stronger linkage between domestic and foreign prices in the same market than under inter-industry trade. So there is a greater degree of PTM under intra-industry trade. Compared to the measures of other determinants, such as market shares, marginal costs and international trade costs which require detailed data for many countries, an intra-industry trade index is relatively straightforward and can be computed from readily available trade data.

Moreover, intra-industry trade indices not only circumvent the difficulty of measuring the market structure of exporting markets in the international context, but also carry some related information about market structure. Imperfect competition due to product differentiation and economy of scales are the main factors to explain increasing intra-industry trade in the last 30 years. So industries with high level of intra-industry trade (IIT) index may have high degree of product differentiation, high level of R\&D investment with large barriers to entry, and 
possibly high level of economy of scales; thus firms in these industries may have larger market power than those in other industries with low level of intra-industry trade. In addition, intra-industry trade index differentiates trade between developed countries and that between developed and developing economies, which in a sense indicates differences in destination markets as argued in Bugamelli and Tedeschi (2008). So we use the index to proxy different trade patterns and market power. The larger the value of the IIT index, the greater the market power of firms in the industry. According to the exchange rate pass-through literature, such as Dornbusch (1987), exchange rate pass-through should be lower in industries with significant market power, so exports in intra-industry trade sectors with greater market power are less likely to be negatively affected by exchange rate movements, because exporters' PTM behavior stabilizes their overseas sales. Thus, we can use the IIT type index to test whether industries with high IIT levels exhibit PTM and hence are less affected by exchange rate fluctuations. If we can find a significant influence of the IIT measures on the impact of exchange rate changes on export decisions, we can partly explain industry heterogeneity.

\subsection{Computing indicators of intra-industry trade and data sources}

We use three intra-industry trade indices, measured at the 3-digit industry level, as our measures of trade pattern and market power to reflect the degree of firms' PTM in each industry. The first one is the Grubel-Lloyd index (Grubel and Lloyd, 1975). The traditional IIT index measures intra-industry trade between UK and all other countries, which is the extent to which simultaneous exports and imports occur within an industry. Formally the IIT index of sector $i$ in country $j$ in period $t$ is defined as: 


$$
I I T_{i, t}^{j}=1-\frac{\left|E X_{i, t}^{j}-I M_{i, t}^{j}\right|}{\left(E X_{i, t}^{j}+I M_{i, t}^{j}\right)}
$$

where $E X_{i, t}^{\prime}$ and $I M_{i, t}^{i}$ represent sectoral exports and imports respectively. A large value of the IIT index is interpreted as a high degree of intra-industry trade and large market power.

It is widely recognized that most countries may specialize in a given commodity with different quality within the same industry. Accordingly, measures of intraindustry trade can be further split into horizontal IIT (where goods are differentiated by attributes with similar quality) and vertical IIT (where goods are differentiated by quality). As stated in Greenaway, Hine and Milner (1995), "vertical IIT can be related more to traditional theories of comparative advantage whilst horizontal IIT falls much more within the remit of 'modern' theories of trade." We expect that horizontal IIT would better capture the difference between two trade patterns (inter-industry vs. intra-industry trade). In addition, market power is likely to be greater in industries with higher levels of horizontal IIT between developed countries with high level investment on R\&D. We, therefore, further proxy market power and trade pattern, as well as identify industries with horizontally differentiated goods, by IIT type trade indices between the UK and other developed countries. Here we assume that industries where the UK exhibits the most intensive trade with developed countries are more likely to include horizontal goods than industries trading with developing countries. It is generally the case that intra-industry trade between developed countries has higher proportion of horizontal IIT than trade between north and south. Although Greenaway, Hine and Milner (1995) and Greenaway, Milner and Elliott (1999) 
show that vertical IIT appears to be more important than horizontal in the UK's trade with its EU partners, their data does confirm that trade of horizontally differentiated goods dominates in the trade between UK and other developed countries, compared to that between UK and developing countries. ${ }^{5}$ So our assumption is plausible. We therefore regard the trade indices between UK and other developed countries as proxies of larger market power and a potentially better proxy for intra-industry trade pattern compared to the traditional IIT index. The second measure of intra-industry trade proxy is the horizontal IIT index of sector $i$ in country $j$ in period $t$ defined as:

$$
I I T-\text { developed } d_{i, t}^{j}=1-\frac{\left|E X_{i,{ }^{j}}^{d^{\prime}}-I M_{i, l}^{d^{d}}\right|}{\left(E X_{i, l}^{j^{d}}+I M_{i, l}^{j^{d}}\right)}
$$

where $E X_{i, I^{d}}{ }^{\mathrm{d}}$ and $I M_{i, 1}{ }^{\mathrm{d}}$ represent sectoral exports and imports with developed countries respectively. IIT-developed is a proxy for horizontal IIT. A high value of the IIT-developed index is interpreted as a high degree of intra-industry trade and a high level of elasticity of substitution among differentiated but similar products. We expect that IIT-developed may be better than IIT to proxy for intra-industry trade pattern and market power.

The above proxies of PMT measure UK domestic market structure. However, UK exporters' PTM behavior is related to their market power in foreign markets. We, therefore, also calculate IIT for international markets to proxy for market power

\footnotetext{
${ }^{5}$ They decompose a Grubel-Lloyd index to disentangle vertical and horizontal IIT. Fontagne, Freudenberg and Gaulier (2006) use another method by categorizing trade flows and compute the share of each category in total trade. Both methods use unit values as the proxy for quality of traded products. Here we do not use the methods to disentangle two types of IIT to proxy for elasticity of substitution between varieties.
} 
and intra-industry trade pattern in international markets. The Grubel and Lloyd type index of sector $i$ in international markets in period $t$ is as follows:

$$
\text { foreignIIT }_{i, t}=1-\frac{\sum_{j=1}^{n}\left|E X_{i, t}^{j}-I M_{i, t}^{j}\right|}{\sum_{j=1}^{n}\left(E X_{i, l}^{j}+I M_{i, t}^{j}\right)}
$$

where $E X_{i, t}^{i}$ and $I M_{i, t}^{i}$ represent sectoral exports and imports for each OECD member with other developed countries respectively. The index is a trade-weighted average of the industry index for the set of trade partners (OECD members). A high value of the IIT index is interpreted as suggesting greater market power due to product differentiation and a higher degree of intra-industry trade compared to low values of the index.

Finally, we follow Bernard, Jensen and Schott (2002) by generating two indices developed countries import and export penetration indices. They use the developed country penetration ratios to "identify industries with relatively high international trade in horizontally differentiated varieties". However, the motivation why these measures are expressed as the ratios is not explained in the paper. Since high international trade in horizontally differentiated varieties may imply large market power for firms in the industry, here we regard and employ these two indices as extra measures and use them to compare to our main IIT measures. The fourth and fifth measures are proxies for trade in horizontally differentiated varieties. The import and export penetration indices of industry $i$ for each year are defined as:

$$
P M_{i}=\frac{m_{i}^{d}}{m_{i}+\text { output } t_{i}-x_{i}}
$$




$$
P X_{i}=\frac{x_{i}^{d}}{\text { output }_{i}}
$$

Where $P M_{i}$ and $P X_{i}$ are developed country import penetration index and export penetration respectively, $m_{i}$ and $x_{i}$ are the value of total imports and exports for the industry, $m_{i}^{d}$ and $x_{i}^{d}$ are the value of imports and exports from and to developed countries, output $t_{i}$ is the total domestic output in the industry in the UK. The higher the penetration index, the higher the level of market power.

The necessary data for imports, exports, bilateral trade data with developed countries, total outputs, and imports and exports of OECD countries ${ }^{6}$ with developed countries for each 3-digit industry are all taken from UNIDO Industrial Demand-Supply Balance Database 2006 which uses the classification of 4-digit ISIC Rev.3 covering the period of 1990-2004. Since our sample period is from 1988, data for 1988 and 1989 is imputed with linear extrapolation. In converting 4digit ISIC Rev.3 to UK SIC (2003) code, there are several industries aggregated: 171 and $172 ; 176$ and $177 ; 181$ and $182 ; 263$ and $264 ; 271,272$ and 273 . Owing to missing data for some industries, we end up with IIT indices for 91 3-digit industries, IIT-developed for 90 industries, foreignIIT for 90, PM indices for 96 , and PX indices for 91.

Table 2 report the mean distribution for IIT, IIT-developed, PM, and PX in 3-digit industry level. IIT type indices generally have higher mean value than penetration ratios. In order to have a sense of the IIT index, we recalculate the IIT index in 2-

\footnotetext{
6 countries are excluded: Iceland, New Zealand, Luxembourg, and Switzerland due to data unavailability; UK is excluded as it is regarded as domestic market.
} 
digit industry level and the statistics for each industry are reported in Table 3. Industries 29, 31, 33 have the highest IIT index, whereas Industries 16, 19, 21 have the lowest. Industries 16 and 18 have the largest standard deviation, whereas Industries 31 and 33 are the most stable industries with the least standard deviation. Figure 1 shows the pattern of IIT in 2-digit industry level. The abrupt trend break for Industry 16 in this figure is a bit strange but true. Since this industry is small, we don't worry too much about that it would play a role to affect our results.

Table 1: Mean Distribution for UK IIT, PM and PX

\begin{tabular}{|c|c|c|c|c|}
\hline $\begin{array}{c}\text { Mean Number of } \\
\text { range }\end{array}$ & IIT & $\begin{array}{c}\text { IIT- } \\
\text { developed }\end{array}$ & PM & PX \\
\hline$>0.8$ & 39 & 49 & 0 & 1 \\
\hline $0.6-0.8$ & 31 & 25 & 4 & 2 \\
\hline $0.4-0.6$ & 10 & 10 & 22 & 17 \\
\hline $0.2-0.4$ & 7 & 6 & 31 & 31 \\
\hline$<0.2$ & 2 & 1 & 33 & 39 \\
\hline
\end{tabular}

Figure 1: UK Intra-Industry Trade Index (2 digit industry level)
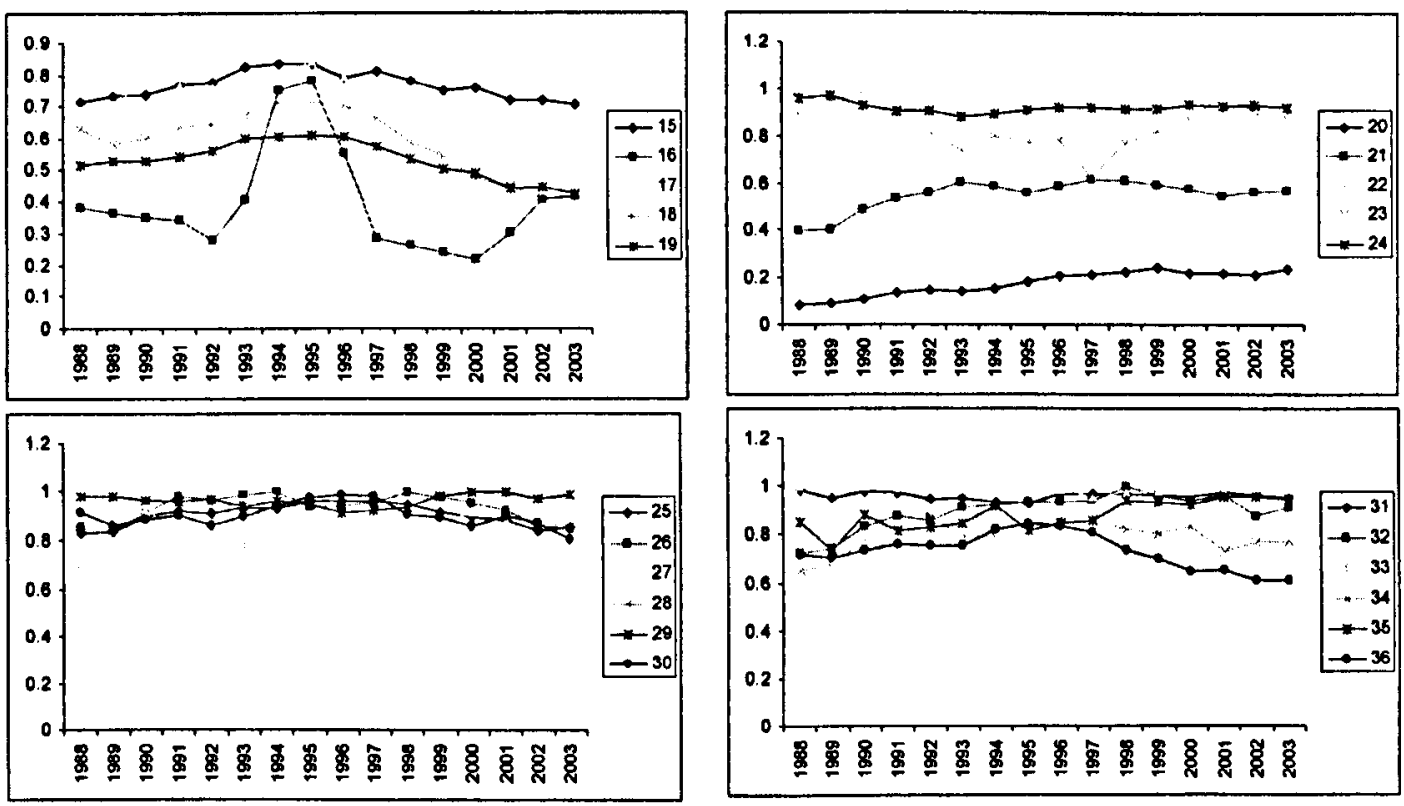
Table 2: Statistics for UK IIT (2 digit industry level)

\begin{tabular}{|c|c|c|}
\hline $\begin{array}{c}\text { Industry } \\
\text { Code }\end{array}$ & Mean & SD \\
\hline 15 & 0.765 & 0.044 \\
\hline 16 & 0.395 & 0.166 \\
\hline 17 & 0.741 & 0.055 \\
\hline 18 & 0.588 & 0.103 \\
\hline 19 & 0.530 & 0.060 \\
\hline 20 & 0.176 & 0.053 \\
\hline 21 & 0.547 & 0.066 \\
\hline 22 & 0.901 & 0.059 \\
\hline 23 & 0.841 & 0.096 \\
\hline 24 & 0.921 & 0.022 \\
\hline 25 & 0.899 & 0.044 \\
\hline 26 & 0.927 & 0.054 \\
\hline 27 & 0.853 & 0.060 \\
\hline 28 & 0.923 & 0.050 \\
\hline 29 & 0.956 & 0.027 \\
\hline 30 & 0.896 & 0.049 \\
\hline 31 & 0.955 & 0.017 \\
\hline 32 & 0.890 & 0.076 \\
\hline 33 & 0.980 & 0.011 \\
\hline 34 & 0.795 & 0.073 \\
\hline 35 & 0.872 & 0.063 \\
\hline 36 & 0.728 & 0.073 \\
\hline $\begin{array}{c}\text { Mean } \\
\text { Distribution } \\
\text { (mean range and }\end{array}$ & $>0.95$ & $29,31,33$ \\
\cline { 2 - 3 } 2 -digit industry \\
code) & $<0.55$ & $16,19,21$ \\
\cline { 2 - 3 } & & \\
\hline & & $24,26,28$ \\
\hline
\end{tabular}

\section{Methodology to Detect Interaction Effects}

In order to examine the role of PTM, we need to detect whether the impacts of exchange rate changes on exports decisions would be influenced by the value of IIT type indices. The standard way to examine the interaction effects (also called moderated relationship) between an independent variable $X$ and a moderator variable $\mathrm{Y}$ on a dependant variable $\mathrm{Z}$ is to add both the independent variable $\mathrm{X}$ and the moderator variable $\mathrm{Y}$, together with the product of the two $\mathrm{XY}$, into the same regression as in Equation (1).

$$
Z=\alpha_{0}+\alpha_{1} X+\alpha_{2} Y+\alpha_{3} X Y+\varepsilon_{1}
$$


In other words, we need to identify whether the nature of the relationship between exchange rates $(\mathrm{X})$ and export decisions $(\mathrm{Z})$ varies at different value of IIT type indices $(\mathrm{Y})$. However, in our current context, there is a high degree of collinearity between the product term and its constituent parts, which might lead to misleading results. Column 1 in Table 4 shows that the correlation between the interaction term and export REER is not high, whereas those between the interaction term and IIT measures are very high in Column 2: with all but one above 0.90 .

Table 3: Correlation between Interaction Term and Each of Its Constituent Parts (Original vs. Centering Counter Part)

\begin{tabular}{|c|c|c|c|c|c|}
\hline \multicolumn{3}{|c|}{ (1) Original variables } & \multicolumn{3}{c|}{$\begin{array}{c}\text { (2) Variables after Centering } \\
\text { transformation }\end{array}$} \\
\hline & REER & IIT measure & & cREER & cIITmeasure \\
\hline REER*IIT & 0.3884 & 0.9114 & cREER* ${ }^{*}$ cIT & -0.0314 & 0.0110 \\
\hline $\begin{array}{c}\text { REER*IIT- } \\
\text { developed }\end{array}$ & 0.4452 & 0.9024 & $\begin{array}{c}\text { cREER*cIIT- } \\
\text { developed }\end{array}$ & -0.0247 & -0.0775 \\
\hline $\begin{array}{c}\text { REER* } \\
\text { foreignIIT }\end{array}$ & 0.5792 & 0.8679 & $\begin{array}{c}\text { cREER* } \\
\text { cforeignIIT }\end{array}$ & -0.0200 & 0.0343 \\
\hline REER*PX & 0.1986 & 0.9932 & cREER*cPX & -0.0949 & 0.2645 \\
\hline REER*PM & 0.1757 & 0.9928 & cREER*cPM & -0.1195 & 0.2296 \\
\hline
\end{tabular}

Note: ${ }^{*}$ is the notation for the multiplication sign.

A possible solution might be to drop the constituent part which is highly correlated with the product term. In our context, this would imply keeping the export REER index and the interaction terms in regressions but dropping the IIT indices to avoid multicollinearity. A problem with this approach, however, is that the interaction terms may now just capture the direct role of IIT indices. This may lead to 
difficulty in explaining interaction effects. ${ }^{7}$ Columns 1 and 3 in Table 5 report the results by just adding interaction terms between export REER and IIT and those between REER and IIT-developed indices in the Heckman selection model respectively. The coefficients for interaction terms are positive and strongly significant in the export participation but not in the export share decision. However, the significant coefficients for the interaction terms can be explained in another way: the results may just imply that IIT type indices have a direct impact on firms export decisions. To see this, the results in Columns 2 and 4 of Table 5, which add the IIT indices only, show similar effects as those using interaction terms. This implies that the interaction terms are actually capturing the direct effects of the IIT type indices on exports. Applying the same methods using the PM and PX measures yields similar conclusions. Columns 1 and 3 in Table 6 report the results using interaction terms only and Columns 2 and 4 report those using penetration ratios only. All the interaction terms seem to capture the direct effects of the IIT type indices or penetration ratios on exports.

Another commonly used method to detect interaction effects is to generate dummy variables according to different levels of the moderator variable (such as high vs. low level of IIT type indices) and interact the dummy with the independent variable $\mathrm{X}$ to see if there is any significant difference in the effect of $\mathrm{X}$ on dependent variable $\mathrm{Z}$ at different selected levels of the moderator variable $\mathrm{Y}$. This kind of interaction contrasts the effect of an independent variable on the dependent variable at one level of the moderator variable with the corresponding effect at another level.

\footnotetext{
${ }^{7}$ Moreover, dropping the constituent term would induce omitted variable bias. As stated in Brambor, Clark and Golder (2006), multicollinearity should not be the reason to omit any of the constituent term during the analysis of interaction effects. They also point out that "the problem of multicollinearity in multiplicative interaction models has been overstated".
} 
Table 4: Heckman Selection Model (MLE): Intra-industry Trade Indices

\begin{tabular}{|c|c|c|c|c|c|c|c|c|}
\hline & \multicolumn{2}{|c|}{$\begin{array}{c}\text { (1) IIT } \\
\text { (interact with REER) }\end{array}$} & \multicolumn{2}{|c|}{$\begin{array}{c}\text { (2) IIT } \\
\text { ( direct role) }\end{array}$} & \multicolumn{2}{|c|}{$\begin{array}{l}\text { (3) IIT-developed } \\
\text { (interact with REER) }\end{array}$} & \multicolumn{2}{|c|}{$\begin{array}{l}\text { (4) IIT-developed } \\
\text { (direct role) }\end{array}$} \\
\hline & $\begin{array}{l}\text { Export } \\
\text { Dummy }\end{array}$ & $\begin{array}{l}\text { Export } \\
\text { Share }\end{array}$ & $\begin{array}{l}\text { Export } \\
\text { Dummy }\end{array}$ & $\begin{array}{l}\text { Export } \\
\text { Share }\end{array}$ & $\begin{array}{l}\text { Export } \\
\text { Dummy }\end{array}$ & $\begin{array}{l}\text { Export } \\
\text { Share }\end{array}$ & $\begin{array}{l}\text { Export } \\
\text { Dummy }\end{array}$ & $\begin{array}{l}\text { Export } \\
\text { Share }\end{array}$ \\
\hline $\begin{array}{l}\text { Lag Export } \\
\text { dummy }\end{array}$ & $\begin{array}{c}3.049 \\
(39.07) \\
* * *\end{array}$ & & $\begin{array}{c}3.049 \\
(39.07) \\
* * *\end{array}$ & & $\begin{array}{c}3.05 \\
(38.95) \\
* * *\end{array}$ & & $\begin{array}{c}3.05 \\
(38.96) \\
* * *\end{array}$ & \\
\hline $\begin{array}{l}\text { Lag } \\
\text { Industry } \\
\text { REER }\end{array}$ & $\begin{array}{c}-0.0024 \\
(-0.40)\end{array}$ & $\begin{array}{c}-0.005 \\
(-2.43) \\
* *\end{array}$ & $\begin{array}{l}-0.0055 \\
(-0.09)\end{array}$ & $\begin{array}{c}-0.004 \\
(-2.43) \\
* *\end{array}$ & $\begin{array}{c}-0.0024 \\
(-0.38)\end{array}$ & $\begin{array}{c}-0.004 \\
(-2.13) \\
* *\end{array}$ & $\begin{array}{l}0.0028 \\
(0.05)\end{array}$ & $\begin{array}{c}-0.004 \\
(-1.93) \\
*\end{array}$ \\
\hline $\begin{array}{l}\text { REER* } \\
\text { IIT }\end{array}$ & $\begin{array}{c}0.0025 \\
(2.46) \\
* *\end{array}$ & $\begin{array}{l}0.0010 \\
(1.75)^{*}\end{array}$ & & & & & & \\
\hline IIT & & & $\begin{array}{c}0.296 \\
(2.39) \\
* *\end{array}$ & $\begin{array}{l}0.116 \\
(1.61)\end{array}$ & & & & \\
\hline $\begin{array}{l}\text { REER* } \\
\text { IIT- } \\
\text { developed }\end{array}$ & & & & & $\begin{array}{l}0.0035 \\
(3.15) \\
* * *\end{array}$ & $\begin{array}{l}0.0008 \\
(1.08)\end{array}$ & & \\
\hline $\begin{array}{l}\text { IIT- } \\
\text { developed }\end{array}$ & & & & & & & $\begin{array}{c}0.401 \\
(3.07) \\
* * * \\
\end{array}$ & $\begin{array}{c}0.08 \\
(0.95)\end{array}$ \\
\hline $\begin{array}{l}\text { Lag log of } \\
\text { emp. }\end{array}$ & $\begin{array}{c}0.0433 \\
(2.26)^{* *}\end{array}$ & $\begin{array}{l}0.0015 \\
(0.32)\end{array}$ & $\begin{array}{c}0.0433 \\
(2.26)^{* *}\end{array}$ & $\begin{array}{l}0.0015 \\
(0.31)\end{array}$ & $\begin{array}{c}0.045 \\
(2.32)^{* *}\end{array}$ & $\begin{array}{l}0.0006 \\
(0.14)\end{array}$ & $\begin{array}{c}0.045 \\
(2.32)^{* *}\end{array}$ & $\begin{array}{l}0.0006 \\
(0.12)\end{array}$ \\
\hline $\begin{array}{l}\text { Lag log of } \\
\text { wage }\end{array}$ & $\begin{array}{l}0.049 \\
(0.96)\end{array}$ & $\begin{array}{c}0.0959 \\
(2.98) \\
* * * \\
\end{array}$ & $\begin{array}{l}0.049 \\
(0.96)\end{array}$ & $\begin{array}{c}0.096 \\
(2.98) \\
* * * \\
\end{array}$ & $\begin{array}{c}0.54 \\
(1.04)\end{array}$ & $\begin{array}{c}0.1006 \\
(3.07) \\
* * * \\
\end{array}$ & $\begin{array}{c}0.54 \\
(1.04)\end{array}$ & $\begin{array}{c}0.1007 \\
(3.07) \\
* * *\end{array}$ \\
\hline $\begin{array}{l}\text { Lag log of } \\
\text { labor } \\
\text { productive. }\end{array}$ & $\begin{array}{l}0.033 \\
(1.02)\end{array}$ & $\begin{array}{c}-0.01 \\
(-0.96)\end{array}$ & $\begin{array}{l}0.033 \\
(1.02)\end{array}$ & $\begin{array}{l}-0.01 \\
(-0.96)\end{array}$ & $\begin{array}{l}0.034 \\
(1.05)\end{array}$ & $\begin{array}{l}-0.12 \\
(-1.2)\end{array}$ & $\begin{array}{l}0.034 \\
(1.04)\end{array}$ & $\begin{array}{l}-0.12 \\
(-1.2)\end{array}$ \\
\hline $\begin{array}{l}\text { Lag log of } \\
\text { age }\end{array}$ & $\begin{array}{l}-0.0239 \\
(-1.50)\end{array}$ & $\begin{array}{c}-0.010 \\
(-2.48) \\
* *\end{array}$ & $\begin{array}{l}-0.024 \\
(-1.50)\end{array}$ & $\begin{array}{c}-0.010 \\
(-2.48) \\
* * \\
\end{array}$ & $\begin{array}{l}-0.0245 \\
(-1.52)\end{array}$ & $\begin{array}{c}-0.010 \\
(-2.50) \\
* *\end{array}$ & $\begin{array}{l}-0.0245 \\
(-1.52)\end{array}$ & $\begin{array}{c}-0.010 \\
(-2.50) \\
* *\end{array}$ \\
\hline $\begin{array}{l}\text { Foreign } \\
\text { owner } \\
\text { dummy }\end{array}$ & $\begin{array}{l}0.1315 \\
(4.08) \\
* * *\end{array}$ & $\begin{array}{c}0.055 \\
(6.70) \\
* * *\end{array}$ & $\begin{array}{l}0.1316 \\
(4.08) \\
* * *\end{array}$ & $\begin{array}{l}0.055 \\
(6.71) \\
* * *\end{array}$ & $\begin{array}{c}0.127 \\
(3.90) \\
* * *\end{array}$ & $\begin{array}{l}0.055 \\
(6.55) \\
* * *\end{array}$ & $\begin{array}{l}0.127 \\
(3.91) \\
* * *\end{array}$ & $\begin{array}{c}0.055 \\
(6.56) \\
* * *\end{array}$ \\
\hline $\begin{array}{c}\text { Lambda } \\
\text { (std. error) }\end{array}$ & \multicolumn{2}{|c|}{$\begin{array}{c}-0.0349 \\
(0.0058)^{* * *}\end{array}$} & \multicolumn{2}{|c|}{$\begin{array}{c}-0.035 \\
(0.0058)^{* * *} \\
\end{array}$} & \multicolumn{2}{|c|}{$\begin{array}{c}-0.346 \\
(0.0058)^{* * *}\end{array}$} & \multicolumn{2}{|c|}{$\begin{array}{c}-0.346 \\
(0.0059)^{* * *}\end{array}$} \\
\hline $\begin{array}{c}\text { Rho } \\
\text { (std. error) }\end{array}$ & \multicolumn{2}{|c|}{$\begin{array}{c}-0.1374 \\
(0.021)^{* * *}\end{array}$} & \multicolumn{2}{|c|}{$\begin{array}{c}-0.1375 \\
(0.021)^{* * *}\end{array}$} & \multicolumn{2}{|c|}{$\begin{array}{c}-0.136 \\
(0.022)^{* * *}\end{array}$} & \multicolumn{2}{|c|}{$\begin{array}{c}-0.136 \\
(0.022)^{* * *}\end{array}$} \\
\hline Observ. & \multicolumn{2}{|c|}{42,165} & \multicolumn{2}{|c|}{42,165} & \multicolumn{2}{|c|}{41,608} & \multicolumn{2}{|c|}{41,608} \\
\hline
\end{tabular}

(i) $\mathrm{Z}$ statistics in parentheses, robust standard errors adjusted for 83 clusters in 3-digit industries.

(ii) *significant at $10 \%$;* significant at $5 \%$; ** significant at $1 \%$

(iii) $p$ is the estimated correlation between the error terms of the two equations; if it is different from zero it suggests that the two equations are related and that the selection model is appropriate; $\lambda$ is the estimated coefficients of the inverse Mills ratio; if it is different from zero it suggests that there is sample selection. 
Table 5: Heckman Selection Model (MLE): Penetration Ratios

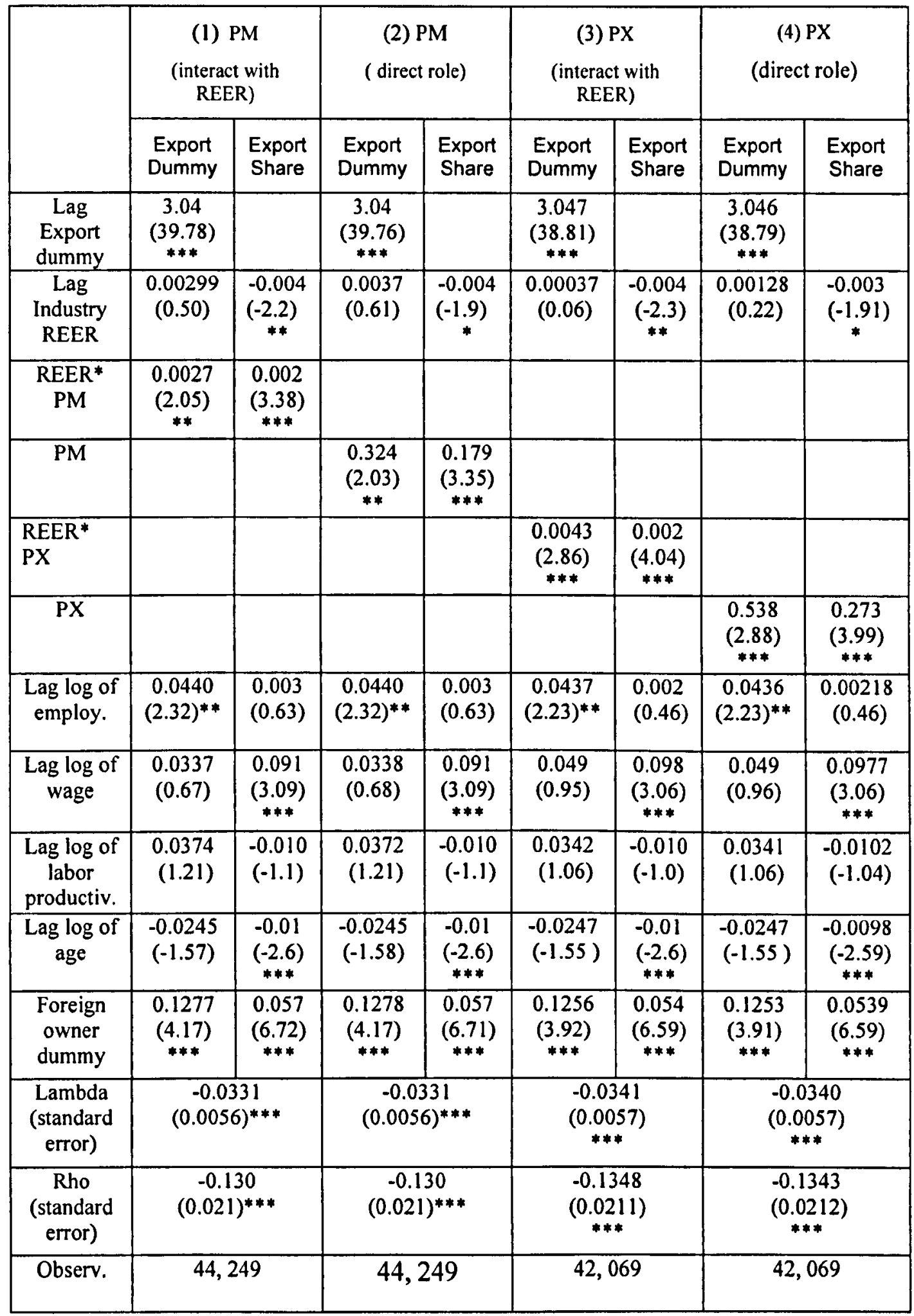

Notes: See notes for Table 5

Usually the moderator variable can be categorized into two or three level: high vs. low; or high, median and low. Dummies are generated according to the categories 
and are interacted with the independent variable. The significance of the interaction terms may imply the existence of interaction effects. We also use this method to examine the interaction effects of PTM on the relationship between exchange rate changes and export decisions.

Some papers (such as Cronbach 1987, Dunlap and Kemery 1987, and Jaccard, Wan and Turrisi 1990) suggest a way to deal with multicollinearity. For a regression as Equation (1), if $X Y$ is highly correlated with $X$ or $Y$, we can make a transformation called mean centering by subtracting the sample mean of each variable: $\mathrm{X}$ and $\mathrm{Y}$. Denote the lowercase letters $x=X-\mu_{X}, y=Y-\mu_{Y}$, the transferred regression becomes:

$$
Z=\beta_{0}+\beta_{1} x+\beta_{2} y+\beta_{3} x y+\varepsilon_{2}
$$

It is almost certain that there is little correlation between $x y$ and either of the constituent part: $x$ or $y$, and Equations (1) and (2) are interchangeable. As argued in Wooldridge (2005), Equation (2) is easier to interpret: $\alpha_{1}$ in the uncentered model (Equation 1) captures the marginal effect of a one-unit increase in $\mathrm{X}$ when $\mathrm{Y}$ is zero (which may not be of much interest in many cases), while the equivalent coefficient $\beta_{1}$ in the centered model (Equation 2) captures the marginal effect of a one-unit increase in $\mathrm{x}$ (the same as a unit increase in $\mathrm{X}$ ) when $\mathrm{Y}$ is at its mean (which is a meaningful value). Column 2 in Table 2 shows that, for our data, the correlations between the centered product term and either of the centered constituent part are quite low: below 0.08 for three IIT measures, and below 0.27 for the two penetration ratios. Compared to the correlations between the original variables, the transformation seems to avoid the problem of multicollinearity. 
Recent papers (such as Brambor, Clark and Golder, 2006; and Braumoeller 2004) argue that this centered model is just an algebraic transformation of the uncentered model from earlier where $\alpha_{0}=\beta_{0}-\beta_{1} \mu_{X}-\beta_{2} \mu_{Y}+\beta_{3} \mu_{X} \mu_{Y}, \alpha_{1}=\beta_{1}-\beta_{3} \mu_{Y}$, $\alpha_{2}=\beta_{2}-\beta_{3} \mu_{X}$, and $\alpha_{3}=\beta_{3}$. It is true that the algebraic transformation that results from centering the variables will result in different coefficients and standard errors in the centered model. However, this is because they measure different substantive quantities in each model and not because one model produces better estimates than the other. As long as there is an interaction effect, the values of the coefficients $\beta_{1}$ and $\beta_{2}$ can be manipulated by subtracting some other constant instead of the sample mean from the original constituent parts. However, the $t$ statistic and coefficient for the interaction term are always unchanged. Examining the significance of the interaction term using either form would be fine, as multicollinearity may not be a big problem for interaction analysis. Since in our sample, the value of REER and IIT type indices for all observations are always nonzero, centering transformation is useful for interpretation. We will use the transformation in our empirical analysis to detect the interaction effects.

\section{Results}

We add 3-digit industry level intra-industry trade measures: IIT, IIT-developed, foreignIIT, $\mathrm{PX}$ and $\mathrm{PM}$, in our Heckman selection regression respectively to examine the role of PTM and the interacting effects of trade patterns, as well as market power, on the relationship between the level of export REER and export decisions. 
Firstly we use dummy variables according to different levels of determinants of PTM and interact them with REER. An interaction term is composed by REER and a dummy variable called "high determinant of PTM", which is 1 if the measure for PTM is high (i.e. lying above a certain percentile). The coefficient of the interaction terms captures the marginal effect of REER on export for industries with a high level of determinants of PTM. Hence, for such industries, the impact on export decision stemming for a change in REER will be given by the sum of the coefficients for REER and the interaction term. If we can find evidence that industries with higher levels of PTM are not less affected by exchange rate changes and are different from those with lower level, we can say that there exists some interacting effect between determinants of PTM and exchange rate movements.

In Row 1 of Table 7, we define an industry as high level of PTM if its IIT type indices are lying at or above the $75^{\text {th }}$ percentile and interact the dummy with REER. The results are reported in Table 7 Row 1. We focus on the coefficients of the interaction terms since we are interested in difference effects of REER in different level of PTM. The coefficient for the interaction term is negative and significant in export entry when using IIT, which implies that industries with higher IIT index are more likely to be negatively affected by exchange rate in export entry decision. This result is unexpected. However, since the coefficient for REER in export entry is always insignificant, we can say there is no interaction effect between REER and IIT in export entry decision. Using IIT-developed, we find the coefficient for the interaction term is positive and weakly significant in the export intensity decision, but insignificant in that for export entry. The results support the hypothesis that firms in industries with high level of PTM are less affected by exchange rate 
movements in their export intensity decision. Coefficients using foreignIIT are always insignificant in both export decisions. When we compare the different results using IIT and IIT-developed, we find a possible explanation for the negative coefficient of IIT dummy interaction term: compared to IIT-developed, IIT index has a composition of vertical intra-industry trade, which might lead to the negative coefficient.

We undertake several exercises to establish the robustness of our findings. Firstly, we apply a break at the 50th percentile to define high level of PTM. Row 2 in Table 7 reports the results using different cut-off dummy for high PTM. Focusing on the coefficients for the interaction terms, Using IIT-developed $\left(75^{\text {th }}\right.$ percentile and $50^{\text {th }}$ percentile), we find the coefficient for the interaction term is positive and significant in the export intensity decision, but insignificant in export entry. The results support the hypothesis that firms in industries with high PTM are less affected by exchange rate movement when they make their export intensity decision. When we use 50 percentile as the cut-off for $I I T$, the interaction term becomes insignificant in both export entry and export intensity decisions. This may imply that the negative coefficient in Row 1 is not robust. Using foreignIIT $\left(75^{\text {th }}\right.$ percentile and $50^{\text {th }}$ percentile), we always find insignificant coefficients for the interaction terms in export entry as well as in export intensity. Results from interacting high PTM dummy using PM and PX $\left(75^{\text {th }}\right.$ percentile) with REER are reported in Row 3. Generally, there is little evidence of the interaction effects using the penetration ratios as measures for PTM, as the coefficients for the interaction terms are insignificant. (Quadratic terms of the measures are included as PM and PX exhibit non-linear role in affecting export decisions.) 
Table 6: Heckman Selection Model (MLE): Interacting Dummy for High PTM

\begin{tabular}{|c|c|c|c|c|c|}
\hline & \multirow[t]{2}{*}{ Measure } & \multicolumn{2}{|c|}{ Export Dummy } & \multicolumn{2}{|c|}{ Export Share } \\
\hline & & $\begin{array}{l}\text { Interaction } \\
\text { term }\end{array}$ & Measure & $\begin{array}{l}\text { Interaction } \\
\text { term }\end{array}$ & Measure \\
\hline \multirow{3}{*}{$\begin{array}{l}\text { (1) } 75^{\text {th }} \\
\text { percentile } \\
\text { as } \\
\text { dummy }\end{array}$} & IIT & $\begin{array}{c}-0.0068 \\
(-2.30)^{* *}\end{array}$ & $\begin{array}{c}0.417 \\
(2.72)^{* * *}\end{array}$ & $\begin{array}{c}-0.0002 \\
(-0.17)\end{array}$ & $\begin{array}{c}0.119 \\
(1.65)^{*}\end{array}$ \\
\hline & $\begin{array}{c}\text { IIT- } \\
\text { developed }\end{array}$ & $\begin{array}{c}-0.00003 \\
(-0.11)\end{array}$ & $\begin{array}{c}0.408 \\
(2.72)^{* * *}\end{array}$ & $\begin{array}{l}0.00016 \\
(1.84)^{*}\end{array}$ & $\begin{array}{c}0.05 \\
(0.52)\end{array}$ \\
\hline & foreignIIT & $\begin{array}{l}0.0004 \\
(1.02)\end{array}$ & $\begin{array}{c}0.47 \\
(1.34)\end{array}$ & $\begin{array}{l}-0.0001 \\
(-0.65)\end{array}$ & $\begin{array}{l}-0.084 \\
(-0.52)\end{array}$ \\
\hline \multirow{3}{*}{$\begin{array}{l}\text { (2) } 50^{\text {th }} \\
\text { percentile } \\
\text { as } \\
\text { dummy }\end{array}$} & IIT & $\begin{array}{c}-0.00026 \\
(-0.61)\end{array}$ & $\begin{array}{c}0.368 \\
(1.83)^{*}\end{array}$ & $\begin{array}{c}1.40 \\
(0.162)\end{array}$ & $\begin{array}{c}0.93 \\
(0.352)\end{array}$ \\
\hline & $\begin{array}{c}\text { IIT- } \\
\text { developed }\end{array}$ & $\begin{array}{l}-0.004 \\
(-0.75)\end{array}$ & $\begin{array}{c}0.56 \\
(2.14)^{* *}\end{array}$ & $\begin{array}{l}0.00028 \\
(1.81)^{*}\end{array}$ & $\begin{array}{l}-0.052 \\
(-0.55)\end{array}$ \\
\hline & foreignIIT & $\begin{array}{c}0.0002 \\
(0.37)\end{array}$ & $\begin{array}{l}0.499 \\
(1.34)\end{array}$ & $\begin{array}{l}0.0001 \\
(0.85)\end{array}$ & $\begin{array}{c}-0.17 \\
(-1.36)\end{array}$ \\
\hline \multirow{2}{*}{$\begin{array}{l}\text { (3) } 75^{\text {th }} \\
\text { percentile } \\
\text { as } \\
\text { dummy }\end{array}$} & $\begin{array}{c}\text { PM } \\
\text { (quadratic } \\
\text { term included) }\end{array}$ & $\begin{array}{c}-0.0009 \\
(-1.58)\end{array}$ & $\begin{array}{c}0.967 \\
(2.78)^{* * *}\end{array}$ & $\begin{array}{l}0.0001 \\
(0.62)\end{array}$ & $\begin{array}{c}0.238 \\
(2.27)^{* *}\end{array}$ \\
\hline & $\begin{array}{c}\text { PX } \\
\text { (quadratic } \\
\text { term included) }\end{array}$ & $\begin{array}{c}-0.001 \\
(-1.74)^{*}\end{array}$ & $\begin{array}{c}2.26 \\
(4.65)^{* * *}\end{array}$ & $\begin{array}{c}-0.00004 \\
(-0.18)\end{array}$ & $\begin{array}{c}0.465 \\
(2.53)^{* *}\end{array}$ \\
\hline
\end{tabular}

Notes: See notes for Table 5

We then add two interaction terms to examine the interaction effects. One is composed by interacting REER with a high PTM dummy $\left(75^{\text {th }}\right.$ percentile), the other by interacting REER with a low PTM dummy (below $25^{\text {th }}$ percentile). Thus the differences in the effects between two pairs of subsamples (top group and the reference group in the middle, and the bottom group and the reference group) can be shown in the same regression. Only IIT, IIT-developed and foreignIIT are used in this case and the results are shown in Table 8. The coefficients for low PTM interaction terms are always insignificant. Results using IIT show that only the high PTM interaction term is negative and significant in export entry. Using IIT- 
developed, we still have positive and significant interacting effects in export intensity for industries with high PTM. Using foreignIIT, the interaction terms are always insignificant both with high and low level dummy interaction terms. For industries with lowest PTM, there are no significant effects. Generally, results generated by interacting dummies for different levels of measures of PTM with REER provide weak evidence for the hypothesis of PTM.

Table 7: Heckman Selection Model (MLE): Interacting with 2 Dummies for High and Low PTM

\begin{tabular}{|c|c|c|c|c|c|c|}
\hline & \multicolumn{2}{|c|}{ (1) IIT } & \multicolumn{2}{|c|}{ (2) IIT-developed } & \multicolumn{2}{|c|}{ (3) foreignIIT } \\
\hline & $\begin{array}{l}\text { Export } \\
\text { Dummy }\end{array}$ & $\begin{array}{l}\text { Export } \\
\text { Share }\end{array}$ & $\begin{array}{l}\text { Export } \\
\text { Dummy }\end{array}$ & $\begin{array}{l}\text { Export } \\
\text { Share }\end{array}$ & $\begin{array}{l}\text { Export } \\
\text { Dummy }\end{array}$ & $\begin{array}{l}\text { Export } \\
\text { Share }\end{array}$ \\
\hline $\begin{array}{l}\text { Lag Industry } \\
\text { REER }\end{array}$ & $\begin{array}{c}0.00008 \\
(0.01)\end{array}$ & $\begin{array}{c}-0.004 \\
(-2.02)^{* *}\end{array}$ & $\begin{array}{l}0.00036 \\
(0.06)\end{array}$ & $\begin{array}{c}-0.004 \\
(-1.98)^{* *}\end{array}$ & $\begin{array}{l}-0.001 \\
(-0.17)\end{array}$ & $\begin{array}{l}-.00355 \\
(-2.18)^{* *}\end{array}$ \\
\hline $\begin{array}{l}\text { REER* Dummy } \\
\text { ( top quartile IIT) }\end{array}$ & $\begin{array}{l}-0.00065 \\
(-2.12)^{* *} \\
\end{array}$ & $\begin{array}{l}0.0000 \\
(0.05)\end{array}$ & & & & \\
\hline $\begin{array}{l}\text { REER* Dummy } \\
\text { (bottom quartile } \\
\text { IIT) }\end{array}$ & $\begin{array}{c}-0.000197 \\
(-0.44)\end{array}$ & $\begin{array}{l}-0.0001 \\
(-0.67)\end{array}$ & & & & \\
\hline IIT & $\begin{array}{l}0.352 \\
(1.57)\end{array}$ & $\begin{array}{l}0.081 \\
(1.12)\end{array}$ & & & & \\
\hline $\begin{array}{l}\text { REER* Dummy } \\
\text { ( top quartile } \\
\text { IIT-developed) }\end{array}$ & & & $\begin{array}{c}-0.00046 \\
(-0.17)\end{array}$ & $\begin{array}{l}0.0002 \\
(1.85)^{*}\end{array}$ & & \\
\hline $\begin{array}{l}\text { REER* Dummy } \\
\text { (bottom quartile } \\
\text { IIT-developed) }\end{array}$ & & & $\begin{array}{c}0.00019 \\
(0.48)\end{array}$ & $\begin{array}{l}-0.0001 \\
(-0.55)\end{array}$ & & \\
\hline IIT-developed & & & $\begin{array}{c}0.467 \\
(2.23)^{* *}\end{array}$ & $\begin{array}{l}0.016 \\
(0.18)\end{array}$ & & \\
\hline $\begin{array}{l}\text { REER* Dummy } \\
\text { ( top quartile } \\
\text { foreignIIT) }\end{array}$ & & & & & $\begin{array}{l}0.0004 \\
(0.94)\end{array}$ & $\begin{array}{l}-0.0001 \\
(-1.07)\end{array}$ \\
\hline $\begin{array}{l}\text { REER* Dummy } \\
\text { (bottom quartile } \\
\text { foreignIIT) }\end{array}$ & & & & & $\begin{array}{l}0.002 \\
(0.21)\end{array}$ & $\begin{array}{l}0.0002 \\
(0.52)\end{array}$ \\
\hline foreignIIT & & & & & $\begin{array}{l}0.558 \\
(1.43)\end{array}$ & $\begin{array}{c}0.01 \\
(0.12)\end{array}$ \\
\hline Observations & \multicolumn{2}{|c|}{42,165} & \multicolumn{2}{|c|}{41,608} & \multicolumn{2}{|c|}{41,633} \\
\hline
\end{tabular}

Notes: See notes for Table 5. ${ }^{*}$ in the column for variable name is the notation for multiplication signal. 
Finally, we report the results by adding the interaction term between REER and each of the continuous measures of determinants of PTM. Column 1 in Table 9 reports the results using IIT as the measure and adding the product of REER and IIT as the interaction term. The coefficient for the interaction term is only positive and significant at $10 \%$ in export share decision. The coefficients for IIT are always insignificant. However, the partial effect of IIT on the export decision is also jointly determined by the interaction term. So it is too early to say that there is little direct role of IIT on export decisions. We then use centering transformation to rerun the regression; the results are reported in Column 2 of Table 9. The coefficients for the interaction term are identical to those using the original form of the variables. However, the coefficients for IIT are different: it is significant in export entry, which implies that firms in industries with a high level of IIT are more likely to export. Results using IIT as proxy for trade pattern and market power provide weak evidence for the interaction effect on the impact of exchange rate changes on export intensity decision of firms: firms in high value of IIT industries are less affected by exchange rate movements in their export intensity decision, whereas there is little evidence for the interaction effect in export entry. We also find that firms in high IIT industries are more likely to enter export markets.

We then use IIT-developed, a proxy disentangling horizontal IIT and vertical IIT, as the proxy to investigate the interaction effect. The results using the original form of variables and the centering transformation are reported in Columns 3 and 4 respectively. In Column 3, the coefficient for IIT-developed is negative and weakly significant in export intensity using the original form of variables, which is 
Table 8: Interaction Effects: Intra-industry Trade Type Indices

\begin{tabular}{|c|c|c|c|c|c|c|c|c|}
\hline & \multicolumn{2}{|c|}{ (1) measure: IIT } & \multicolumn{2}{|c|}{$\begin{array}{l}\text { (2) measure: IIT } \\
\text { (centering) }\end{array}$} & \multicolumn{2}{|c|}{$\begin{array}{l}\text { (3) measure: } \\
\text { IIT-developed }\end{array}$} & \multicolumn{2}{|c|}{$\begin{array}{l}\text { (4) measure: IIT- } \\
\text { developed } \\
\text { (centering) }\end{array}$} \\
\hline & $\begin{array}{l}\text { Export } \\
\text { Dummy }\end{array}$ & $\begin{array}{l}\text { Export } \\
\text { Share }\end{array}$ & $\begin{array}{l}\text { Export } \\
\text { Dummy }\end{array}$ & $\begin{array}{l}\text { Export } \\
\text { Share }\end{array}$ & $\begin{array}{l}\text { Export } \\
\text { Dummy }\end{array}$ & $\begin{array}{l}\text { Export } \\
\text { Share }\end{array}$ & $\begin{array}{l}\text { Export } \\
\text { Dummy }\end{array}$ & $\begin{array}{l}\text { Export } \\
\text { Share }\end{array}$ \\
\hline $\begin{array}{c}\text { Lag } \\
\text { Export } \\
\text { dummy }\end{array}$ & $\begin{array}{c}3.049 \\
(39.06) \\
* * *\end{array}$ & & $\begin{array}{c}3.049 \\
(39.06) \\
* * *\end{array}$ & & $\begin{array}{c}3.05 \\
(38.93) \\
* * *\end{array}$ & & $\begin{array}{c}3.05 \\
(38.93) \\
* * *\end{array}$ & \\
\hline $\begin{array}{l}\text { Lag } \\
\text { Industry } \\
\text { REER }\end{array}$ & $\begin{array}{l}-0.0035 \\
(-0.41)\end{array}$ & $\begin{array}{c}-0.008 \\
(-2.78) \\
* * *\end{array}$ & & & $\begin{array}{l}-0.006 \\
(-0.68)\end{array}$ & $\begin{array}{c}-0.01 \\
(-2.81) \\
* * *\end{array}$ & & \\
\hline IIT & $\begin{array}{l}-0.181 \\
(-0.17)\end{array}$ & $\begin{array}{l}-0.523 \\
(-1.29)\end{array}$ & & & $\begin{array}{l}-0.541 \\
(-0.53)\end{array}$ & $\begin{array}{l}-0.814 \\
(-1.8)^{*}\end{array}$ & & \\
\hline $\begin{array}{l}\text { REER* } \\
\text { measure }\end{array}$ & $\begin{array}{l}0.004 \\
(0.45)\end{array}$ & $\begin{array}{l}0.0055 \\
(1.69)^{*}\end{array}$ & & & $\begin{array}{l}0.008 \\
(0.92)\end{array}$ & $\begin{array}{c}0.0078 \\
(2.07) \\
* *\end{array}$ & & \\
\hline cREER & & & $\begin{array}{c}-0.0001 \\
(-0.02)\end{array}$ & $\begin{array}{c}-0.004 \\
(-1.98) \\
* *\end{array}$ & & & $\begin{array}{l}0.001 \\
(0.17)\end{array}$ & $\begin{array}{l}-0.003 \\
(-1.8)^{*}\end{array}$ \\
\hline cmeasure & & & $\begin{array}{c}0.294 \\
(2.38)^{* *}\end{array}$ & $\begin{array}{l}0.117 \\
(1.17)\end{array}$ & & & $\begin{array}{c}0.406 \\
(3.13) \\
* * *\end{array}$ & $\begin{array}{l}0.099 \\
(1.28)\end{array}$ \\
\hline $\begin{array}{l}\text { cREER* } \\
\text { cmeasure }\end{array}$ & & & $\begin{array}{l}0.004 \\
(0.45)\end{array}$ & $\begin{array}{l}0.0055 \\
(1.69)^{*}\end{array}$ & & & $\begin{array}{l}0.008 \\
(0.92)\end{array}$ & $\begin{array}{c}0.0078 \\
(2.07) \\
* *\end{array}$ \\
\hline $\begin{array}{l}\text { Foreign } \\
\text { owner } \\
\text { dummy }\end{array}$ & $\begin{array}{c}0.1314 \\
(4.08) \\
* * *\end{array}$ & $\begin{array}{c}0.055 \\
(6.67) \\
* * *\end{array}$ & $\begin{array}{c}0.1314 \\
(4.08) \\
* * *\end{array}$ & $\begin{array}{c}0.055 \\
(6.67) \\
* * *\end{array}$ & $\begin{array}{c}0.127 \\
(3.90) \\
* * *\end{array}$ & $\begin{array}{c}0.055 \\
(6.53) \\
* * *\end{array}$ & $\begin{array}{c}0.127 \\
(3.90) \\
* * *\end{array}$ & $\begin{array}{c}0.055 \\
(6.53) \\
* * *\end{array}$ \\
\hline $\begin{array}{l}\text { Lambda } \\
\text { (standard } \\
\text { error) }\end{array}$ & \multicolumn{2}{|c|}{$\begin{array}{c}-0.035 \\
(0.0057) \\
* * *\end{array}$} & \multicolumn{2}{|c|}{$\begin{array}{c}-0.035 \\
(0.0057) \\
* * *\end{array}$} & \multicolumn{2}{|c|}{$\begin{array}{c}-0.346 \\
(0.0058)^{* * *}\end{array}$} & \multicolumn{2}{|c|}{$\begin{array}{c}-0.346 \\
(0.0058)^{* * *}\end{array}$} \\
\hline $\begin{array}{l}\text { Rho } \\
\text { (standard } \\
\text { error) }\end{array}$ & \multicolumn{2}{|c|}{$\begin{array}{c}-0.1374 \\
(0.021) \\
* * *\end{array}$} & \multicolumn{2}{|c|}{$\begin{array}{c}-0.1374 \\
(0.021) \\
* * *\end{array}$} & \multicolumn{2}{|c|}{$\begin{array}{c}-0.136 \\
(0.022)^{* * *}\end{array}$} & \multicolumn{2}{|c|}{$\begin{array}{c}-0.136 \\
(0.022)^{* * *}\end{array}$} \\
\hline Observ. & \multicolumn{2}{|c|}{42,165} & \multicolumn{2}{|c|}{42,165} & \multicolumn{2}{|c|}{41,608} & \multicolumn{2}{|c|}{41,608} \\
\hline
\end{tabular}

Notes: cREER: centered REER; cmeasure: centered measure. See notes in Table 8 for other notes.

counter-intuitive. However, it becomes insignificant in Column 4 when we use mean centered variables. Generally, the evidence for the direct role of IITdeveloped is similar to that using IIT: firms in industries with high levels of IIT are more likely to enter international markets. Turing to the interaction effect, now the interaction term in export intensity decision is positive and significant at $5 \%$, which is stronger than that using IIT. There is no evidence for an interaction effect in the 
regression for export entry as before. As discussed earlier, since IIT-developed index is more likely to disentangle horizontal IIT from vertical IIT whereas IIT index includes both types of intra-industry trade, IIT-developed better captures the spirit of different trade patterns and market power. Thus, it is not surprising that the significance of the interaction term using IIT-developed is stronger than that using IIT, if there is an interaction effect of PTM on the relationship between exchange rates and exports.

As we discussed in Section 3, PTM is a phenomenon in international markets and a measure for international market may be more plausible. The previous two IIT type measures are related to the case of UK itself, whereas such a measure should be related to the outside of UK which is the international market environment for UK exporting firms. So we also use the intra-industry trade measure for international markets to examine interaction effects. This is the third measure in Section 4: foreignIIT which may mainly capture horizontal IIT in north-north trade. Column 1 in Table 10 reports the results. Row (a) reports the results using the original form; Row (b) presents the results using the centering transformation. The coefficient for the interaction term is now positive and strongly significant, both in export entry and export intensity decisions. The results strongly support the hypothesis of PCM: UK firms in industry with a high average level of IIT within developed countries are less likely negatively affected by exchange rate changes in their export intensity and positively affected in export entry.

The same methods are applied using PX and PM as proxies for the determinants of PTM (Columns 2 and 3 in Table 10 report the relevant results). The coefficients for 
Table 9: Interaction Effects: Foreign IIT and Penetration Ratios

\begin{tabular}{|c|c|c|c|c|c|c|c|}
\hline & \multicolumn{2}{|c|}{ (1) measure: foreignIIT } & \multicolumn{2}{|c|}{ (2) measure: PX } & \multicolumn{2}{|c|}{ (3) measure: PM } \\
\hline & & $\begin{array}{l}\text { Export } \\
\text { Dummy }\end{array}$ & $\begin{array}{l}\text { Export } \\
\text { Share }\end{array}$ & $\begin{array}{l}\text { Export } \\
\text { Dummy }\end{array}$ & $\begin{array}{l}\text { Export } \\
\text { Share }\end{array}$ & $\begin{array}{l}\text { Export } \\
\text { Dummy }\end{array}$ & $\begin{array}{l}\text { Export } \\
\text { Share }\end{array}$ \\
\hline \multirow[b]{3}{*}{ a } & REER & $\begin{array}{c}-0.019 \\
(-1.93)^{*}\end{array}$ & $\begin{array}{c}-0.009 \\
(-3.85)^{* * *}\end{array}$ & $\begin{array}{c}0.004 \\
(0.66) \\
\end{array}$ & $\begin{array}{c}-0.003 \\
(-1.89)^{*}\end{array}$ & $\begin{array}{l}0.0045 \\
(0.71)\end{array}$ & $\begin{array}{c}-0.004 \\
(-1.90)^{*}\end{array}$ \\
\hline & measure & $\begin{array}{c}-2.487 \\
(-1.68)^{*}\end{array}$ & $\begin{array}{c}-1.00 \\
(-2.71)^{* * *}\end{array}$ & $\begin{array}{c}2.205 \\
(2.06)^{* *}\end{array}$ & $\begin{array}{l}0.259 \\
(0.69)\end{array}$ & $\begin{array}{l}0.739 \\
(0.94)\end{array}$ & $\begin{array}{l}0.067 \\
(0.19)\end{array}$ \\
\hline & $\begin{array}{l}\text { REER* } \\
\text { measure }\end{array}$ & $\begin{array}{c}0.026 \\
(2.10)^{* *}\end{array}$ & $\begin{array}{c}0.0076 \\
(2.90)^{* * *}\end{array}$ & $\begin{array}{c}-0.014 \\
(-1.65)^{*}\end{array}$ & $\begin{array}{l}0.0001 \\
(0.04)\end{array}$ & $\begin{array}{l}-0.0035 \\
(-0.56)\end{array}$ & $\begin{array}{l}0.0009 \\
(0.33)\end{array}$ \\
\hline \multirow[b]{3}{*}{ b } & cREER & $\begin{array}{c}-0.0003 \\
(-0.05)\end{array}$ & $\begin{array}{c}-0.0038 \\
(-2.31)^{* *}\end{array}$ & $\begin{array}{l}0.0007 \\
(0.13)\end{array}$ & $\begin{array}{r}-0.003 \\
(-1.92)^{*}\end{array}$ & $\begin{array}{l}0.004 \\
(0.60)\end{array}$ & $\begin{array}{c}-0.003 \\
(-1.96)^{* *}\end{array}$ \\
\hline & cmeasure & $\begin{array}{c}0.55 \\
(1.74)^{*}\end{array}$ & $\begin{array}{l}-0.116 \\
(-0.80)\end{array}$ & $\begin{array}{c}0.581 \\
(2.93)^{* * *}\end{array}$ & $\begin{array}{c}0.273 \\
(3.83)^{* * *}\end{array}$ & $\begin{array}{c}0.33 \\
(2.02)^{* *}\end{array}$ & $\begin{array}{c}0.177 \\
(3.20)^{* * *}\end{array}$ \\
\hline & $\begin{array}{l}\text { cREER* } \\
\text { cmeasure }\end{array}$ & $\begin{array}{c}0.026 \\
(2.10)^{* *}\end{array}$ & $\begin{array}{c}0.0076 \\
(2.90)^{* * *}\end{array}$ & $\begin{array}{c}-0.014 \\
(-1.65)^{*}\end{array}$ & $\begin{array}{l}0.0001 \\
(0.04)\end{array}$ & $\begin{array}{c}-0.0035 \\
(-0.56)\end{array}$ & $\begin{array}{c}0.0009 \\
(0.33)\end{array}$ \\
\hline \multirow{3}{*}{ c } & cREER & & & $\begin{array}{l}0.001 \\
(0.20)\end{array}$ & $\begin{array}{c}-0.004 \\
(-2.32)^{* *}\end{array}$ & $\begin{array}{l}0.004 \\
(0.74)\end{array}$ & $\begin{array}{c}-0.004 \\
(-2.18)^{* *}\end{array}$ \\
\hline & $\begin{array}{l}\text { cREER* } \\
\text { cmeasure }\end{array}$ & & & $\begin{array}{l}-0.007 \\
(-0.91)\end{array}$ & $\begin{array}{c}-0.0015 \\
(-0.41)\end{array}$ & $\begin{array}{c}-0.0028 \\
(-0.49)\end{array}$ & $\begin{array}{l}0.0008 \\
(0.32)\end{array}$ \\
\hline & $\begin{array}{c}\text { cREER }^{*} \\
\text { cmeasure }^{2}\end{array}$ & & & $\begin{array}{l}0.013 \\
(0.28)\end{array}$ & $\begin{array}{c}0.032 \\
(1.76)^{*}\end{array}$ & $\begin{array}{c}-0.0157 \\
(-0.51)\end{array}$ & $\begin{array}{l}0.011 \\
(1.60)\end{array}$ \\
\hline & $\begin{array}{l}\text { mber of } \\
\text { ervation }\end{array}$ & \multicolumn{2}{|c|}{41633} & \multicolumn{2}{|c|}{42069} & \multicolumn{2}{|c|}{44249} \\
\hline
\end{tabular}

Notes: See notes in Table 9.

the penetration ratios themselves are always positive and significant in both export

entry and export intensity decisions, which implies that industries with high developed country penetration ratios are more likely enter international markets and export more. However, all the coefficients for the interaction terms are insignificant or negative in both export entry and export share decisions. We then try to examine whether there is a nonlinear interaction effect between mean centered REER and mean centered PX (PM) by adding a quadratic interaction term and $\mathrm{PX}^{2}\left(\mathrm{PM}^{2}\right)$ in the regression and the results are reported in Row (c). We only report the coefficients for mean centered REER, the linear interaction term and quadratic interaction term to see the interaction effects. Using PX as the measure, we find among the coefficients for interaction terms, only the quadratic interaction 
term in export intensity equation is positive and weakly significant, which implies that industries with high levels of PX are less likely negatively affected by REER in export intensity decision and this effect is nonlinear. So there is some evidence for the PTM hypothesis. However, we find no significant interaction effect using PM as the measure. Although these penetration ratios try to capture high international trade in horizontally differentiated varieties, they are not intraindustry trade indices and they also carry some information on other potentially important features such as domestic output. The ratios may, therefore, not accurately capture the spirit of the determinants of PTM which relates to market power and different trade patterns. So we don't think the weakly significant and insignificant results using PX and PM would mitigate our evidence for PTM.

\section{Conclusions}

In this chapter, we attempted to explain industry heterogeneity by PTM behaviour. This is, to our knowledge, the first attempt to explain export behaviour under exchange rate fluctuation by PTM and imperfect competition in export markets using firm level data. Firms' PTM behaviour would stabilize exports in terms of local currency and the demand from export markets, which may exhibit less impact from exchange rate changes.

When we used some arbitrarily chosen cut-off dummies to identify the effects of exchange rates on exports in different levels of determinant of PTM, the results are inconclusive. Only for one IIT type index (IIT-developed) do we find weakly significant interaction effects. In contrast, when using a continuous moderator variable (IIT type indices as the proxy for trade pattern and market power), we find 
strongly significant evidence supporting the hypothesis of pricing to market. The results using penetration ratios only provide us with weakly significant or insignificant evidence. Intuitively, IIT type indices better capture the spirit of determinants of PTM than penetration ratios. In general, we find significant evidence for the role of PTM on the relationship between exchange rate changes and export decisions: industries with high level of intra-industry trade and thus high degree of PTM and low level of exchange rate pass-through are less negatively affected by exchange rate changes. 


\section{Chapter 5}

\section{Exchange Rate Uncertainty and Exports}

\section{Introduction}

In investigating the effects of exchange rate variability on firms' export behaviour, it is also important to examine the impact of exchange rate uncertainty, which is the focus of this chapter. The theoretical papers on exchange rate uncertainty-trade relationship and on uncertainty-investment relationship provide a framework for the hysteresis effect of uncertainty. There are many empirical papers focusing on the relationship between exchange rate uncertainty and trade using aggregate data. Empirical evidence from micro data, however, is limited. The evidence for uncertainty-investment is vast using both aggregate and disaggregates data, which provide some implications for our empirical study. In this chapter, we examine empirically the effects of exchange rate uncertainty on firm export decisions using firm level micro data for UK manufacturing firms.

The existing literature suggests several interesting issues: as noted in Carruth et al. (2000a), different measures of uncertainty may have different drawbacks, and may influence the results differently; there are some difficulties in empirically testing for hysteresis effects: it is difficult to directly test the relationship between the unobservable thresholds of entry and exit decisions and uncertainty; existing papers focusing on investigating simple correlations between trade and exchange rate uncertainty may face the problem of model misspecification; the relative scarcity of observations of entry and exit makes it difficult to observe the effects on export entry and exit. 
This chapter aims to contribute in two main respects. One is to test the hypothesis of hysteresis effects of exchange rate uncertainty on firm export behaviour using micro data from UK firms. Theoretical models show that there are hysteresis effects on trade price and quantities. And the size of gap between the exchange rates that trigger entry and exit is increasing with uncertainty. Here we use new measures of exchange rate uncertainty to overcome the difficulties in the empirical test mentioned above and test the hypothesis. Our empirical results provide evidence that increased exchange rate uncertainty increases the inertia in firms export share decision, whereas there is no significant evidence for hysteresis effects in the export participation decision. The other contribution is to examine the impacts of exchange rate uncertainty on multinational firms. We find that multinationals are less likely influenced by exchange rate uncertainty than indigenous firms. We also find some evidence of industry heterogeneity in the exchange rate uncertainty effects on exports, which is consistent with the results from studies of the uncertainty and investment relationship.

The structure of this chapter is as follows: to motivate our empirical analysis, we first review the theoretical literature in Section 2. Section 3 summarizes the existing empirical evidence. Our method for measuring exchange rate uncertainty is introduced in Section 4. We discuss empirical implications from theoretical models and our ways to test the hysteresis hypothesis in Section 5. Section 6 presents the results, and conclusions are set out in Section 7.

\section{Theoretical Background}


The theoretical models describing the effects of exchange rate uncertainty on firm export behavior can be split into two groups. One strand is the literature on the relationship between exchange rate uncertainty and international trade. The other might be drawn from the literature on investment under uncertainty. It has some of the same characteristics as export behavior: incurring sunk costs and requiring investment. Thus, we can reasonably regard the firm's entry into export markets as investment under uncertainty from exchange rates fluctuation.

\subsection{Exchange rate uncertainty and trade}

In the theoretical literature on the impact of exchange rate uncertainty on trade, there is a critical assumption of sunk entry costs that cause the hysteresis effects of exchange rate movements on trade prices and quantities. Baldwin (1988) introduced the idea that large exchange rate swings can cause hysteresis effects on trade prices and quantities when market entry costs are sunk. One limitation of Baldwin (1988) is the assumption of perfect foresight, while Baldwin and Krugman (1989) assume that the levels of real exchange rate at successive instants of time are independently and identically distributed. Though this novel theory clearly shows the role of sunk costs in inducing hysteresis effects on trade, the assumption regarding exchange rate forecasting is restrictive.

Dixit (1989a) assumes that the real exchange rate is a random walk, or more accurately in continuous time, a Brownian motion. Under this critical assumption, the firms' export market entry and exit decisions can be described as options, and thus the techniques and intuition in the area of financial economics can be introduced into the model. In this way, the "real option" emerges as a new cause of 
hysteresis, as greater exchange rate volatility makes entry and exit options more valuable and therefore less readily exercised. The paper shows that the size of the gap between the exchange rates that trigger entry and exit is not constant but an increasing function of the uncertainty around current exchange rates. Of course, if there were literally no sunk costs, there could be no hysteresis; but given some sunk costs, the "option" plays a bigger role.

All of the papers in the literature focus on the entry and exit decisions of firms under uncertainty. However, we are also interested in the effects that exchange rate uncertainty has on export intensity decisions. Since empirical work such as Campa (2004) and Bernard and Jensen (2004b) shows trade adjustment to exchange rate variability comes mainly from the adjustment of existing exporters, examining the effects on export share seems quite important. However, Dixit (1989b) is the only paper using a similar setup to show that quantity hysteresis can occur when the exchange rate follows a continuous-time random walk. In the case with no sunk entry costs, he assumes a flow fixed costs for production. This assumption is realistic, since if there is no fixed cost, the investment need never be abandoned as it can be kept alive at little loss by choosing very small output. The framework to show the quantity hysteresis is just the same as the sunk costs setup in the option approach by substituting fixed costs for sunk costs.

\subsection{Investment under uncertainty}

The literature on investment under uncertainty has grown over the last three decades and is vast. In that literature, Dixit and Pindyck (1994) is a breakthrough. Sunk costs, which cause irreversibility, and option theory, are the two key elements 
in their model with the prediction of a negative impact of uncertainty on investment due to the option value of delaying investment. The idea is similar to that in Dixit (1989b): if there are large sunk costs embedded in new capital investment, uncertainty implying an option value of waiting, the firm is more likely to postpone its investment decisions in the face of increased uncertainty until more information becomes available. Uncertainty will depress investment. In particular, an increase in the volatility of the stochastic process that determines the returns from investment will raise the trigger point. Their theoretical framework dominates all the related empirical work, both at macro and micro level of analysis. The extensions of the investment under uncertainty model are various: some consider the type of returns to scale; some take market structure into consideration; effect on long run equilibrium and short run effect. Different assumptions lead to different arguments of the uncertainty-investment relationship, which provide a broader horizon for empirical work than theoretical models on exchange rate uncertainty and trade.

Moreover, Caballero (1991), Huizinga (1993), Dixit and Pindyck (1994), and Darby et al. (1999) have shown that in certain circumstances uncertainty may still have a positive effect. Darby et al. (1999) extend the Dixit-Pindyck model and show how the impact of exchange-rate uncertainty may vary according to the characteristics of the industrial sector. For some industries uncertainty will depress investment, but for others it would foster it. Industry heterogeneity is a determinant of the effect of uncertainty on investment. The literature shows that the overall impact of uncertainty on investment depends on the relative strength of several critical factors such as industrial structure (i.e. the degree of competition), the type 
of returns to scale, degree of risk aversion, and source of uncertainty itself. The literature thus suggests a problem of aggregation when empirically examining the issue using macro data.

We should note that though the theory of investment under uncertainty can explain the export market entry decision of firms under exchange rate uncertainty, it provide little guidance on export behavior of firms after entry.

\section{Empirical Background}

There are many empirical papers, using macro (see Clarke et al 2004 for a survey) and micro data (such as Campa 2004), examining exchange rate volatility and trade, in which exchange rate volatility is the proxy for exchange rate uncertainty. Table 1 reports a brief summary of the main empirical evidence. Generally, early work finds ambiguous evidence for a negative effect of volatility on trade; recent work using gravity models finds some negative effect; more recent evidence from micro data is ambiguous.

There are many empirical papers investigating the investment decision under uncertainty. The survey by Carruth et al. (2000a) compare the empirical evidence, and shows that the studies using aggregate data usually find a significant negative sign, while the overall empirical results from disaggregated data are less conclusive. Table 2 summarizes some main papers. The results suggest similar evidence to Carruth et al. (2000a): negative evidence from aggregate data, ambiguous evidence from disaggregated data. 
Table 1: Studies of Exchange Rate Uncertainty and Trade Relationship

\begin{tabular}{|c|c|c|c|c|}
\hline Study & Country & Uncertainty proxy & $\begin{array}{l}\text { Estimation } \\
\text { method }\end{array}$ & Results \\
\hline $\begin{array}{l}\text { Bélanger et } \\
\text { al. (1988)* }\end{array}$ & $\begin{array}{l}\text { U.S. export } \\
\text { volumes to } \\
\text { Canada }\end{array}$ & square of forecast error & IVE, GIVE & $\begin{array}{l}\text { significant and } \\
\text { negative in two } \\
\text { out of } 5 \text { sectors }\end{array}$ \\
\hline $\begin{array}{l}\text { Lastrapes } \\
\text { and Koray } \\
(1990)^{*}\end{array}$ & U.S. & $\begin{array}{l}\text { 12-month standard } \\
\text { deviation of growth rate } \\
\text { of real (and } \\
\text { nominal) exchange rate }\end{array}$ & VAR & weak negative \\
\hline $\begin{array}{l}\text { Asseery and } \\
\text { Peel } \\
(1991)^{*}\end{array}$ & $\begin{array}{l}\text { Australia, Japan, } \\
\text { U.K., } \\
\text { U.S., W.G. }\end{array}$ & $\begin{array}{l}\text { squared residual from } \\
\text { ARIMA process fitted to } \\
\text { real exchange rate }\end{array}$ & $\begin{array}{l}\text { error } \\
\text { correction } \\
\text { technique }\end{array}$ & $\begin{array}{l}\text { significant and } \\
\text { positive except } \\
\text { for U.K. }\end{array}$ \\
\hline $\begin{array}{l}\text { Bélanger et } \\
\text { al. (1992)* }\end{array}$ & $\begin{array}{l}\text { U.S. imports } \\
\text { from Canada }\end{array}$ & $\begin{array}{l}2 \text { measures: squared of } \\
\text { forecast error and } \\
\text { nonparametric method to } \\
\text { isolate risk premium in } \\
\text { forecast error }\end{array}$ & IVE, GIVE & not significant \\
\hline $\begin{array}{l}\text { Frankel and } \\
\text { Wei (1993) } \\
\text { * }\end{array}$ & 63 countries & $\begin{array}{l}\text { standard deviation of } \\
\text { first difference of log } \\
\text { of nominal (and real) } \\
\text { exchange rate }\end{array}$ & $\begin{array}{l}\text { Gravity } \\
\text { model, } \\
\text { cross-section } \\
\text { OLS and IV }\end{array}$ & $\begin{array}{l}\text { small negative } \\
\text { in } 1980 \text {, positive } \\
\text { in } 1990\end{array}$ \\
\hline $\begin{array}{l}\text { Dell'Ariccia } \\
\text { (1999) }\end{array}$ & $\begin{array}{l}\text { bilateral trade of } \\
\text { the } 15 \mathrm{EU} \\
\text { members and } \\
\text { Switzerland }\end{array}$ & $\begin{array}{l}4 \text { measures: the std. } \\
\text { deviation, the sum of } \\
\text { squares of the forward } \\
\text { errors, and percentage } \\
\text { difference between the } \\
\text { maximum and the } \\
\text { minimum of the nominal } \\
\text { spot rate }\end{array}$ & $\begin{array}{l}\text { Gravity } \\
\text { model }\end{array}$ & $\begin{array}{l}\text { small but } \\
\text { significantly } \\
\text { negative }\end{array}$ \\
\hline Rose (2000) & 186 countries & the standard deviation & $\begin{array}{l}\text { Gravity } \\
\text { model }\end{array}$ & $\begin{array}{l}\text { small but } \\
\text { significant } \\
\text { negative }\end{array}$ \\
\hline $\begin{array}{l}\text { Cho, } \\
\text { Sheldon and } \\
\text { McCorriston } \\
(2002)\end{array}$ & $\begin{array}{l}\text { Bilateral } \\
\text { agricultural trade } \\
\text { across } 10 \\
\text { developed } \\
\text { countries }\end{array}$ & $\begin{array}{l}\text { the standard deviation } \\
\text { and Peree and Steinherr } \\
\text { measure }\end{array}$ & $\begin{array}{l}\text { Gravity } \\
\text { model }\end{array}$ & $\begin{array}{l}\text { Significant } \\
\text { negative }\end{array}$ \\
\hline \multirow[t]{2}{*}{$\begin{array}{l}\text { Tenreyro } \\
(2003)\end{array}$} & $\begin{array}{l}\text { a broad sample } \\
\text { of countries }\end{array}$ & the standard deviation & $\begin{array}{l}\text { Gravity } \\
\text { model, IV }\end{array}$ & $\begin{array}{l}\text { insignificant } \\
\text { effect }\end{array}$ \\
\hline & & Micro & Data & \\
\hline $\begin{array}{l}\text { Campa } \\
(2004)\end{array}$ & $\begin{array}{l}\text { Spanish } \\
\text { manufacturing } \\
\text { firms }\end{array}$ & GARCH model & $\begin{array}{l}\text { Random } \\
\text { effects probit }\end{array}$ & insignificant \\
\hline $\begin{array}{l}\text { Das, Robert } \\
\text { and Tybout } \\
(2007)\end{array}$ & $\begin{array}{l}\text { Panel data on } \\
\text { three Colombian } \\
\text { manufacturing } \\
\text { industries }\end{array}$ & $\begin{array}{l}\text { Variance of exchange } \\
\text { rate shocks }\end{array}$ & Simulation & $\begin{array}{l}\text { Volitality can } \\
\text { matter, but not } \\
\text { dramatically. } \\
\text { Heterogeneity } \\
\text { across industries }\end{array}$ \\
\hline
\end{tabular}

Summary of the papers marked with * is modified from Côte (1994) to show a rough picture of related studies before 1994. See Côté (1994) for a detailed survey of the literature on exchange rate volatility and trade before 1994 . 
Table 2: Studies of Uncertainty-investment Relationship

\begin{tabular}{|c|c|c|c|c|}
\hline Study & Country & Uncertainty proxy & $\begin{array}{c}\text { Estimation } \\
\text { method }\end{array}$ & Results \\
\hline $\begin{array}{l}\text { Driver and } \\
\text { Moreton } \\
(1991)^{*}\end{array}$ & UK & $\begin{array}{l}\text { Unconditional variance of } \\
\text { ourput and inflation }\end{array}$ & $\mathrm{ECM}$ & negative \\
\hline $\begin{array}{l}\text { Caballero } \\
\text { and Pindyck } \\
\text { (1996) }\end{array}$ & $\begin{array}{l}\text { US } \\
\text { manufacturing } \\
\text { industries }\end{array}$ & Standard deviation of price & $\begin{array}{l}\text { Function of } \\
\text { required return }\end{array}$ & negative \\
\hline $\begin{array}{l}\text { Ferderer } \\
(1993)^{*}\end{array}$ & US & $\begin{array}{l}\text { Risk premium from interest } \\
\text { rate term structure }\end{array}$ & $\begin{array}{l}\text { Jorgensen and } \\
q \text { models }\end{array}$ & negative \\
\hline $\begin{array}{l}\text { Price } \\
(1996)^{*}\end{array}$ & UK & $\begin{array}{l}\text { GARCH estimates of } \\
\text { conditional variance of GDP }\end{array}$ & $\mathrm{ECM}$ & Negative \\
\hline $\begin{array}{l}\text { Carruth, } \\
\text { Dicherson } \\
\text { and Henley } \\
(2000 \mathrm{~b})\end{array}$ & UK & $\begin{array}{l}\text { Gold price and abnormal } \\
\text { return to holding gold }\end{array}$ & ECM & Negative \\
\hline \multirow[t]{2}{*}{$\begin{array}{l}\text { Darby et al } \\
\text { (1999) }\end{array}$} & $\begin{array}{l}\text { France, Italy, } \\
\text { Germany, UK, } \\
\text { US }\end{array}$ & $\begin{array}{l}\text { std. deviation of real } \\
\text { effective Exchange rate }\end{array}$ & ECM & negative \\
\hline & & Disaggregated & Data & \\
\hline $\begin{array}{l}\text { Campa } \\
(1993)^{*}\end{array}$ & $\begin{array}{l}\text { US } 4 \text { digit } \\
\text { panel of FDI } \\
\text { investment }\end{array}$ & $\begin{array}{l}\text { Exchange rate volatility } \\
\text { from ARMA model } \\
\text { residuals }\end{array}$ & Tobit model & Negative \\
\hline $\begin{array}{l}\text { Leahy } \\
\text { and Whited } \\
(1996)^{*}\end{array}$ & $\begin{array}{l}\text { U.S. } \\
\text { manufacturing } \\
\text { firms }\end{array}$ & $\begin{array}{l}\text { Variance of of daily stock } \\
\text { returns, forecasts of } \\
\text { volatility using vector } \\
\text { autoregression }\end{array}$ & VAR, GMM & $\begin{array}{l}\text { Weak } \\
\text { negative/none }\end{array}$ \\
\hline $\begin{array}{l}\text { Henley, } \\
\text { Carruth and } \\
\text { Dickerson } \\
(2003)\end{array}$ & $\begin{array}{l}\text { UK firm level } \\
\text { panel data }\end{array}$ & $\begin{array}{l}\text { Industry-wide: std. } \\
\text { deviation of sector PPI. } \\
\text { Firm-specific uncertainty: } \\
\text { abnormal stock return } \\
\text { volatility }\end{array}$ & $\begin{array}{l}\text { First } \\
\text { difference } \\
\text { Investment } \\
\text { function, IV }\end{array}$ & $\begin{array}{l}\text { firm-specific } \\
\text { uncertainty: } \\
\text { positive } \\
\text { effects ; } \\
\text { industry-wide } \\
\text { uncertainty: } \\
\text { negative } \\
\text { effects }\end{array}$ \\
\hline $\begin{array}{l}\text { Bond and } \\
\text { Cummins } \\
(2004)\end{array}$ & $\begin{array}{l}\text { U.S. } \\
\text { companies }\end{array}$ & $\begin{array}{l}3 \text { measures: the volatility in } \\
\text { the firm's stock returns; } \\
\text { disagreement among } \\
\text { securities analysts in their } \\
\text { forecasts of profits; and the } \\
\text { variance of forecast errors in } \\
\text { profits. }\end{array}$ & GMM & $\begin{array}{l}\text { Significant } \\
\text { negative }\end{array}$ \\
\hline $\begin{array}{l}\text { Bloom, } \\
\text { Bond and } \\
\text { Van Reenen } \\
(2007)\end{array}$ & $\begin{array}{l}\text { UK } \\
\text { manufacturing } \\
\text { companies }\end{array}$ & $\begin{array}{l}\text { standard deviation of daily } \\
\text { stock returns for firm }\end{array}$ & $\mathrm{ECM}$ & $\begin{array}{l}\text { Negative } \\
\text { effects }\end{array}$ \\
\hline Lee $(2005)$ & $\begin{array}{l}\text { South Korea } \\
\text { manufacturing } \\
\text { firms }\end{array}$ & $\begin{array}{l}\text { the standard deviation of } \\
\text { daily stock returns divided } \\
\text { by the standard deviation of } \\
\text { the returns on the market } \\
\text { index }\end{array}$ & $\begin{array}{l}\text { Fixed effects } \\
\text { model }\end{array}$ & $\begin{array}{l}\text { Significant } \\
\text { negative in } \\
\text { post-crisis } \\
\text { period, } \\
\text { insignificant } \\
\text { in pre-crisis }\end{array}$ \\
\hline
\end{tabular}

Summary of the papers marked with * is modified from Carruth, Dickerson and Henley (2000a) to show a rough picture of related studies before 2000 . 
Although the theoretical literature does not provide a direct testable proposition to empirically examine the hysteresis effects, as it is difficult to compute the thresholds of the investment/export entry decision and examine the relationship between the thresholds and uncertainty, the papers give us a rough picture. These models need to be developed further to draw out empirically testable implications. However, in practice, most studies in this area focus on investigating simple correlations of trade volume/ export entry with exchange rate volatility, and rate of investment/ uncertainty. As Dixit and Pindyck (1994, p. 421) argue, the theoretical models cannot be directly tested by investigating simple equilibrium relationships between rates of investment and measures of uncertainty. Investigating simple correlations is "a strategy which is highly questionable since any observed significant relationship may be an artefact of underlying model misspecification."

It is also worth noting that in the literature there is no accepted best way to capture uncertainty: each measure may have some limitations, and since uncertainty is unobservable, we can only employ some proxies. For a survey of the measures of uncertainty see Carruth et al. (2000a). However, some empirical studies such as Carruth et al. (2000a) and Darby et al. (1999) also show that different specifications of the uncertainty make little difference to the numerical results.

Theoretical work suggests industry heterogeneity is a determinant of investment. Little empirical work investigates this however. Henley, Carruth and Dickerson (2003) show that firm-specific uncertainty and industry-wide uncertainty have opposite effects on investment. Das, Robert and Tybout (2007) provide some 
evidence for industry heterogeneity. We will split our sample by industry to examine the issue.

\section{Measuring Exchange Rate Uncertainty}

As indicated by Pagan and Ullah (1988) and Carruth et al. (2000a), it is important to recognize possible potential biases introduced by the use of a measure of risk/uncertainty which is critical in all studies on the role of exchange rate uncertainty. As a proxy, some form of moving average of past standard deviations of spot rates has been widely employed in previous studies.' In this chapter, we argue that a more appropriate measure should be based on the observed forecast errors on the forward market as in Bélanger et al. (1992) and Dell'Ariccia (1999).

Most papers use the canonical conditional variances from GARCH model as in Campa (2004) or the standard deviation of first differences of the logarithmic exchange rate. The latter can difference away the possible constant trend of an exchange rate process, which may be expected and thus should not be included as uncertainty. In the standard theory of Brownian motion in the option approach, uncertainty is modeled as the standard deviation/variance. This measure is also consistent with the theoretical model (see Dixit and Pindyck, 1994). This may be the reason why most papers use standard deviation and variance as the measure for uncertainty. Some earlier papers, such as Bélanger et al. (1992) and Dell'Ariccia (1999), consider the difference between the previous period's forward rate and current spot to be an indicator of exchange rate uncertainty. The percentage difference between the maximum and minimum of the nominal spot rate over a

\footnotetext{
'In the theoretical papers of Baldwin (1988), Baldwin and Krugman (1989) and Dixit (1989b), uncertainty means a risk of mean reversion.
} 
certain period preceding the observation plus a measure of exchange rate misalignment is another measure. This index is more likely to capture medium-run uncertainty, since past large changes may generate expected volatility. As stated in Dell'Ariccia (1999), we should note that the measures of uncertainty mentioned above are backward-looking, as the past volatility is used to predict present risk. There are some other issues that also need to be considered: data frequency, which temporal window and so on.

One of the advantages of measures based on differences between the actual and forward exchange rate, as showed in Dell'Ariccia (1999) is that, "under a target zones regime, or under pegged but adjustable exchange rates, it would pick up the effect of the presence of a 'peso problem' or lack of credibility of the official parity". The measures also take firms' hedge behavior into consideration. When hedging instruments are available, the predicted part of exchange rate volatility can be hedged away and hence may not have much effect on trade. The extent to which exchange rate volatility is a source of uncertainty depends on the degree to which exchange rate movements are predictable. And in the spirit of uncertainty in the investment-uncertainty literature, uncertainty should be the error in a prediction model. This suggests that the appropriate measure should be related to deviations between actual and predicted exchange rates. As the rational expectations literature has stressed, only the unexpected portion of a variable really matters and the forecast error used in this chapter exploits this idea. Moreover, the measures provide us with a novel way to investigate the issue: all other measures of uncertainty are bounded non-negative, whereas we can embed the direction (positive and negative), as well as the size or magnitude, of uncertainty in this type 
of measure, which enables us to obtain more informative results. Of course, by using the forward error, we have automatically restricted our analysis to a nominal environment since there is no readily available forward or future market for the real exchange rate. A nominal analysis could well be justified, in a quarterly context, by assuming that firms are maximizing short-run nominal profits and, consequently, that they are facing only nominal exchange rate uncertainty. We feel, in that context, our measure is appropriate whether traders maximize real or nominal expected profits.

So we measure exchange rate uncertainty using the observed forecast errors given by the difference between appropriately chosen 90 -day forward rates $f_{t-l}$ in the period of $t-1$ and spot exchange rates $s_{i}$ The difference between these two variables $w_{t}=s_{t}-f_{t-I}$ measures unexpected changes in the exchange rate. The use of the difference between current spot and the previous forward rates assumes that hedging is available to each exporter to cover all international transactions and is costless.

Data for spot rates and forward rates for currencies of the UK's main export destinations are needed. Since we have shown in Section 4 of Chapter 2 (as well as the comparison between Figures 2 and 3 in that chapter) that changes of REER in the UK mainly depend on two currencies: Euro (German Mark) and US dollar. We use exchange rate data for these (Euro/GBP and USD/GBP) and compute the weighted average industry specific exchange rate uncertainty by using normalized export weights for the two currency areas in each 3-digit industry. We include China, Hong Kong, Taiwan, Singapore and Canada into the US dollar area as the 
currencies in these areas were pegged the US dollar for most of the period we investigate. The data we use to calculate exchange rate uncertainty is from Datastream. We focus on differences between the spot rate and previous 3 month forward rate. Since the period between placing an order (signing a contract) and receiving payment is usually three months, we follow Bélanger et al. (1992) and Dell'Ariccia (1999) in choosing the 3 month forward rate to compute uncertainty. The data we use is monthly exchange rates (spot and 3 month forward rates) at mid-month (the 13th of each month), expressed as foreign currency per GBP. However, we need annual data for exchange rate uncertainty to be consistent with our annual firm level data. The uncertainty for each year is calculated in two ways: the average of the 12 monthly differences between logarithms of spot rate and logarithms of the 3 month forward rate predicted 3 months earlier (positive if appreciation, negative if depreciation): $\left(\sum w_{1}\right) / 12$ (AVG, hereafter), and the average of the absolute value of the 12 monthly differences: $\left(\sum \mid w_{t}\right) / 12$ (ABS, hereafter). To capture short run uncertainty, we lagged 3 months, i.e. for each year the 12 monthly data is from October of the previous year to September of the current year. The use of average differences is new as no existing literature takes the sign of differences into consideration. Thus, the average of differences (AVG) can be regarded as the main direction of exchange rate uncertainty for each year, and the average of absolute differences (ABS) is the proxy for the size or magnitude of the uncertainty.

We then take a look at the general pattern of uncertainty. Figure 1 shows eight 3digit industry specific exchange rate uncertainty measures calculated in several ways: AVG, ABS, SD and S-Sum, where SD is computed using the conventional 
standard deviation of the first differences of the logarithmic spot exchange rate, S-

Sum is the sum of squares of the differences between the spot rate and forward rate as in Dell'Ariccia (1999). The measures of uncertainty follow roughly the same pattern across industries with small variations in magnitude. The most volatile

\section{Figure 1: 3-digit Industry Specific Exchange Rate Uncertainty}
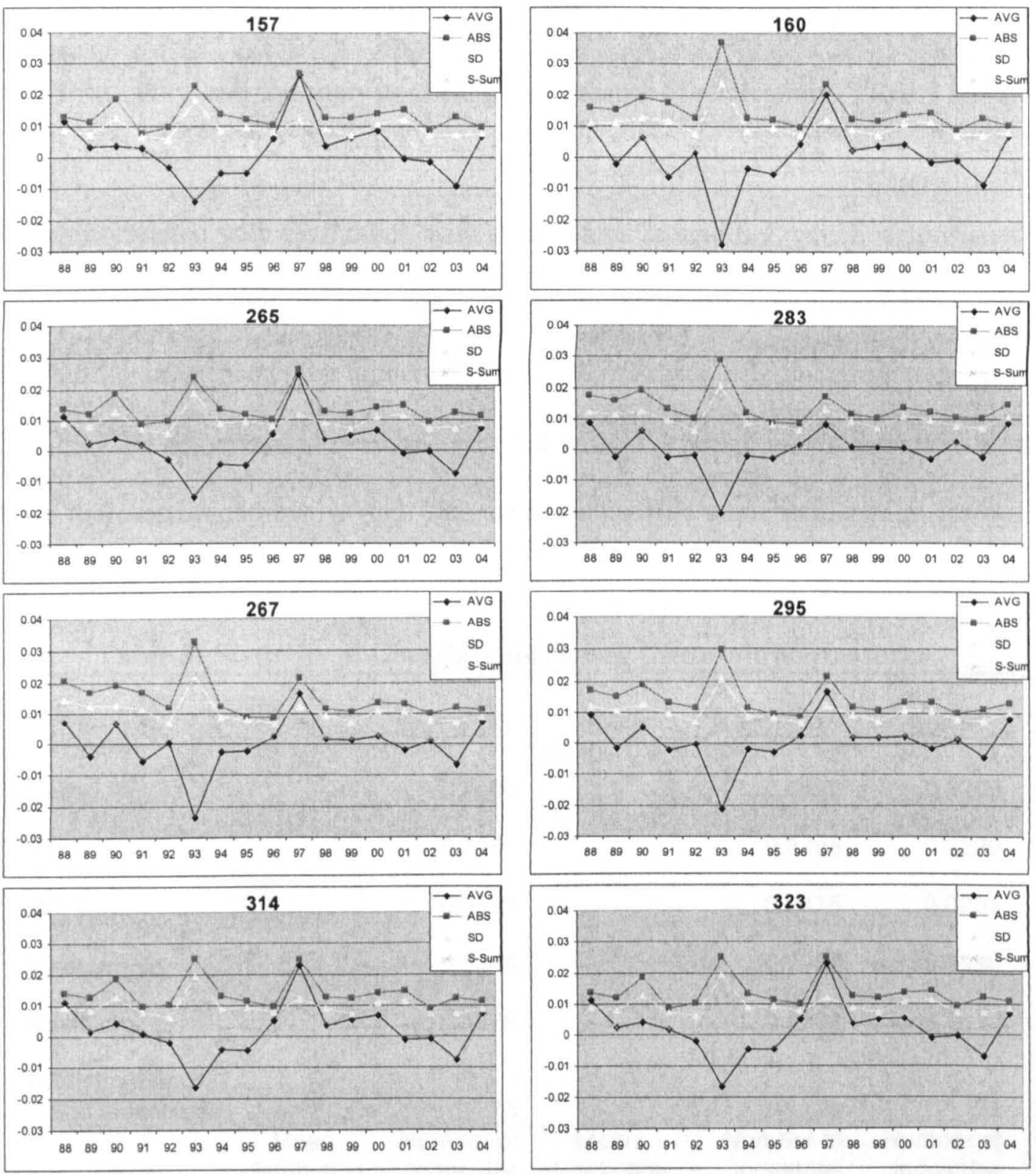

Note:

AVG: the average of the 12 monthly differences between spot rate and forward rate.

ABS: the average of the 12 monthly absolute value of differences between spot rate and forward rate

SD: standard deviation of the 12 monthly first differences of the logarithmic spot exchange rate.

S-Sum: the sum of square of the 12 monthly differences between spot rate and forward rate 
measure is AVG: fluctuating between -0.03 to 0.025 . The second most volatile measure is ABS: moving between 0.01-0.04. S-Sum follows the same pattern as ABS (correlations between 0.94-0.97) with a smaller magnitude, as it is the square of the forecast errors (which is less than 1). The standard measure of uncertainty, $\mathrm{SD}$, also follows the similar pattern of $\mathrm{ABS}$ with smaller magnitude and less volatility. The figure implies that S-Sum may mitigate the magnitude of exchange rate uncertainty, and thus may not be a good measure of uncertainty. Table 3 shows correlations between the measures. The standard measure SD, ABS and S-Sum are highly correlated with each other, with correlations around 0.9 , which is consistent with the argument that different specifications of the uncertainty are correlated and make little difference to the numerical results. AVG has a different correlation with SD: they are negative and weakly correlated with an average of -0.25 .5 industries have the correlations below -0.5 . The statistics provide some explanation for our later results.

Table 3: Statistics of Correlations among Uncertainty Measures

\begin{tabular}{|c|c|c|c|c|c|}
\hline & & AVG/SD & & ABS/SD & $\begin{array}{l}\text { SD/S- } \\
\text { Sum }\end{array}$ \\
\hline Mean & & -0.2500 & & 0.8826 & 0.9208 \\
\hline Median & & -0.2281 & & 0.8788 & 0.9229 \\
\hline Std. Dev. & & 0.0852 & & 0.0475 & 0.0264 \\
\hline Minimum & & -0.5349 & & 0.7934 & 0.8580 \\
\hline Maximum & & -0.1375 & & 0.9768 & 0.9610 \\
\hline $\begin{array}{l}\text { Total number of } \\
\text { 3-digit industries }\end{array}$ & & 78 & & 78 & 78 \\
\hline & $\begin{array}{c}\text { Mean } \\
\text { distribution }\end{array}$ & $\begin{array}{l}\text { Number of } \\
\text { industries }\end{array}$ & $\begin{array}{c}\text { Mean } \\
\text { distribution }\end{array}$ & $\begin{array}{l}\text { Number of } \\
\text { industries }\end{array}$ & $\begin{array}{l}\text { Number of } \\
\text { industries }\end{array}$ \\
\hline & $<-0.4$ & 5 & $>0.9$ & 28 & 62 \\
\hline & $(-0.4,-0.3)$ & 16 & $0.8-0.9$ & 47 & 16 \\
\hline & $>-0.3$ & 57 & $<0.8$ & 3 & 0 \\
\hline
\end{tabular}

Five industries with AVG/SD below $-0.4: 160,191,267,335,362$ 
Comparing the pattern of uncertainty with that for 3-digit industry specific REER and the first differences of REER as shown in Figure 2 (Industry 314 as an example), we find that the uncertainty is quite different from the two series: the peaks and troughs in uncertainty are in 1993 and 1997, whereas those for REER are in 1995 and 1999, and in 1992 and 1996 for the first differences of REER. The comparison clearly shows that exchange rate uncertainty is not correlated with changes of the level of exchange rates.

\section{Figure 2: REER and Its First Difference for Industry 314}
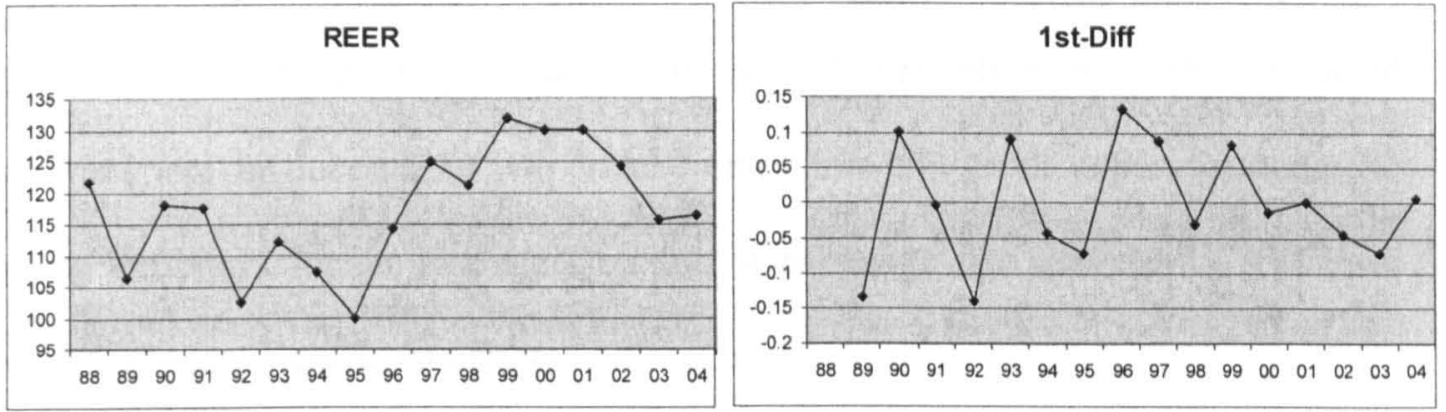

\section{Empirical Implications and Testing for Hysteresis}

We adopt a similar empirical estimation method as in Chapter 2, a two-stage sample selection model, to empirically examine the relationship between exchange rate uncertainty and firms export decisions. This provides an appropriate way to observe both export entry behavior and behavior after entry. The proxy for exchange rate uncertainty is included in both the selection and outcome equations to examine its effects on export participation and on export intensity respectively.

According to the theory, if the persistent band for a firm to invest (the investment threshold) increases with the magnitude of uncertainty, we may expect an insignificant relationship between the magnitude of uncertainty and export entry instead of a negative or positive effect. As the standard measures of uncertainty are 
bounded non-negative, we may regard those measures as proxies for the magnitude of uncertainty. So using the non-negative measures AVG, SD and S-Sum, there should be no significant relationship between each and firms export decisions. If, however, we can separate the different monthly directions (positive and negative) of uncertainty within a period (a year), and the uncertainties for different directions are asymmetric (the magnitude of uncertainty in one direction is averagely larger than that in the other direction during the period), we may observe a significant effect of net uncertainty beyond a symmetric band on investment. In the case of exchange rate uncertainty, a net depreciation/appreciation over the predicted forward rate in uncertainty will induce entry into/exit from export markets. So using the proxy for the average direction of exchange rate uncertainty AVG, we may find some significant effects.

As most empirical papers using micro data do not observe the effects of exchange rate volatility on entry into export markets, which may due to the large sunk entry costs (thus high thresholds) and rarity of observations of entry, we may not observe the effects on export market entry. However, we may observe quantity hysteresis within exporters. As pointed out in Dixit (1989b), in the case with no sunk entry cost, there is a fixed costs for production, and fixed costs play the same role of sunk costs in hysteresis effect. As the fixed costs are much less than the sunk costs, we are more likely to observe hysteresis. we also examine the nonlinearity of the effect. We examine the industry heterogeneity by splitting the sample by industry. Since our data provides information on ownership of firms, we are able to test the hypothesis that multinationals are less likely affected by exchange rate uncertainty as suggested in Clark et al (2004). 


\section{Results}

The results of the sample selection model with uncertainty are shown in Table 4. Column 1 reports the results using AVG, column 2 using ABS. In Column 1, the coefficient of uncertainty is negative and significant in export share, but insignificant in export participation. In Column 2, the coefficient is always negative and insignificant. Although the value of uncertainty using $A B S$ is generally larger than that using AVG, the latter can capture the main direction and pattern of uncertainty after offsetting shocks with different directions in a year, whereas the former cannot. This suggests that the direction of uncertainty has little effect on firm export participation decisions, but has a significant negative effect on the export share decision. The magnitude of the uncertainty has little impact on firm export behavior according to the results in Column 2. However, Dell'Ariccia (1999) on macro data using the sum of squares of the forward errors finds significant and negative effects of exchange rate uncertainty on trade. According to our results, whether or not the uncertainty changes greatly in magnitude, it has little impact on the export behavior of firms. What really matters is the main direction of the asymmetric uncertainty movements. The comparison of the different impacts between average and average absolute uncertainty gives us a new way to examine the role of uncertainty. The results in Column 1 and 2 together provide some evidence for the hypothesis in Dixit (1989a) that the hysteresis gap for firms is not constant but an increasing function of uncertainty.

We also use the standard measure of exchange rate uncertainty SD, standard deviation of the first differences of the logarithmic exchange rate, to examine the effects. Column 3 reports the results. The coefficient for uncertainty is positive and 
Table 4: Heckman Selection Model (MLE): Uncertainty

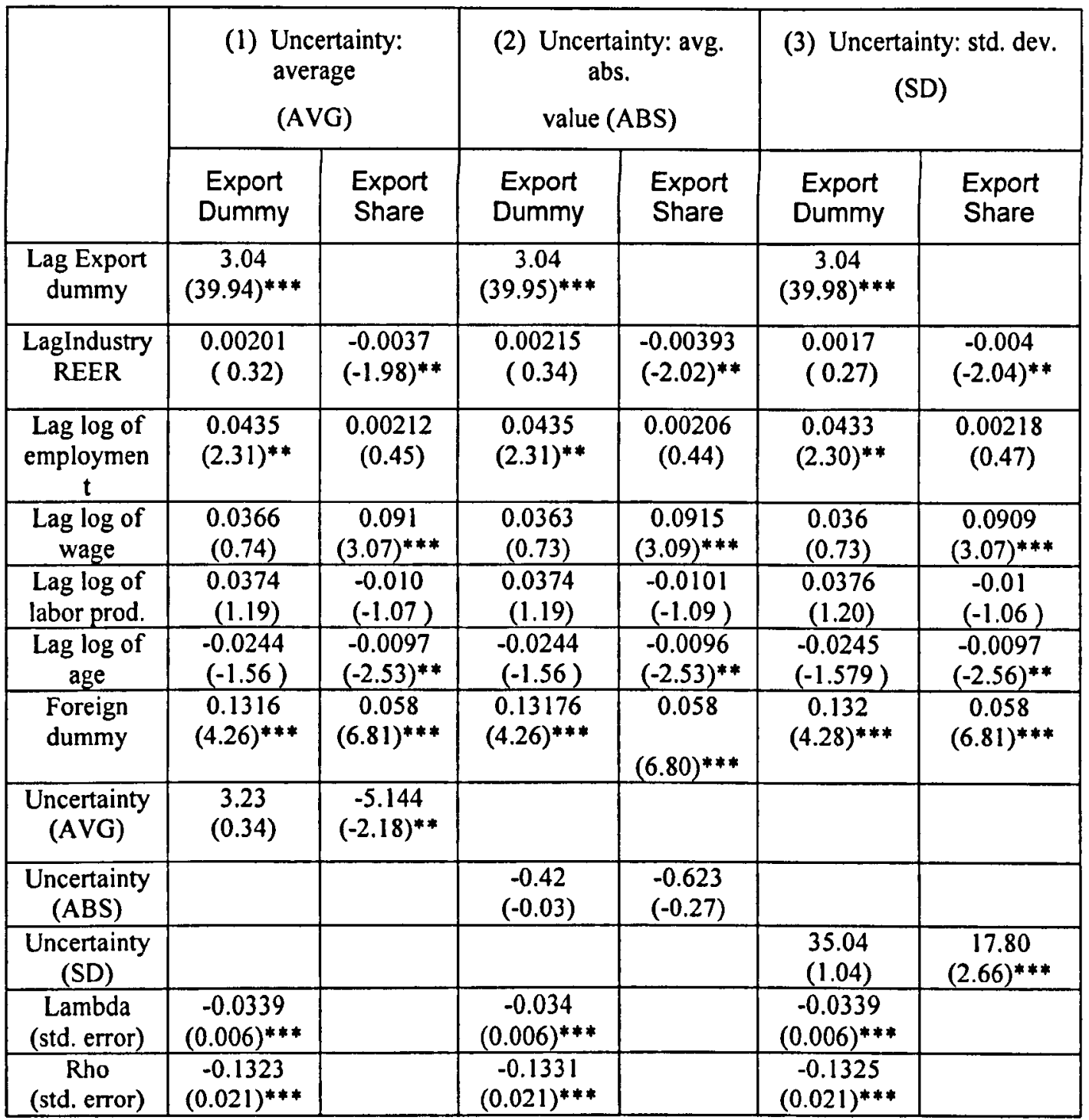

\section{Observations: 44,251 Firms: 5,876}

Notes: (i) $\mathrm{Z}$ statistics in parentheses, robust standard errors adjusted for 83 clusters in 3-digit industries.

(ii) *significant at $10 \%$; ** significant at $5 \%$; *** significant at $1 \%$

(iii) $\rho$ is the estimated correlation between the error terms of the two equations; if it is different from zero it suggests that the two equations are related and that the selection model is appropriate; $\lambda$ is the estimated coefficients of the inverse Mills ratio; if it is different from zero it suggests that there is sample selection.

highly significant in the export intensity equation, which implies a positive effect of uncertainty on export share. Although the theoretical model does show that the effects can be positive in some circumstances, we do not expect that it is a typical phenomenon. As we obtain a negative and significant effect using AVG measure, and AVG and SD are negatively correlated, the positive effects from SD are not 
surprising. Our new measure of exchange rate uncertainty may be a more plausible proxy.

Table 5: Models of Export Participation

\begin{tabular}{ccc}
\hline $\begin{array}{c}\text { Uncertainty } \\
\text { measure }\end{array}$ & $\begin{array}{c}\text { (1)Fixed-effects Linear probability } \\
\text { (t statistic) }\end{array}$ & $\begin{array}{c}\text { (2)Ramdom-effects } \\
\text { Probit (z statistic) }\end{array}$ \\
\hline AVG & 0.5325 & 3.643 \\
& $(0.63)$ & $(0.30)$ \\
\multirow{2}{*}{ ABS } & 1.236 & 3.68 \\
& $(1.03)$ & $(0.43)$ \\
SD & 2.256 & 49.0 \\
& $(0.86)$ & $(1.31)$ \\
\hline indicates significant at $10 \%: * *$ indicates significant $5 \% ; * *$ indicates significant at $1 \%$
\end{tabular}

* indicates significant at $10 \% ;{ }^{* *}$ indicates significant at $5 \% ;{ }^{* * *}$ indicates significant at $1 \%$

To check robustness, we use another estimation methodology to investigate the role of uncertainty. Table 5 reports the results using a linear probability model and random-effects probit model. The coefficients for uncertainty are all insignificant in both cases, which suggests little effect of exchange rate uncertainty on firm export entry decision. The results are consistent with the results of export entry equation in Table 4.

We further investigate whether the effect is nonlinear. Table 6 reports the results when adding the square of measures of the uncertainty in the sample selection model. Only the coefficient of uncertainty using AVG in export share equation is significant. Moreover, the squared term is negative and significant, which suggests that an increase in uncertainty would induce bigger negative effect on export intensity. 
Table 6: Heckman Selection Model: Uncertainty (nonlinear)

\begin{tabular}{|c|c|c|c|c|}
\hline & \multicolumn{2}{|c|}{ (1) uncertainty (AVG) } & \multicolumn{2}{|c|}{ (2) uncertainty (ABS) } \\
\hline & $\begin{array}{l}\text { Export } \\
\text { Dummy }\end{array}$ & $\begin{array}{l}\text { Export } \\
\text { Share }\end{array}$ & $\begin{array}{l}\text { Export } \\
\text { Dummy }\end{array}$ & $\begin{array}{l}\text { Export } \\
\text { Share }\end{array}$ \\
\hline $\begin{array}{l}\text { Lag Export } \\
\text { dummy }\end{array}$ & $\begin{array}{c}3.04 \\
(39.90)^{* * *}\end{array}$ & & $\begin{array}{c}3.04 \\
(40.00)^{* * *}\end{array}$ & \\
\hline $\begin{array}{l}\text { LagIndustry } \\
\text { REER }\end{array}$ & $\begin{array}{r}0.00203 \\
(0.32)\end{array}$ & $\begin{array}{l}-0.00379 \\
(-2.04)^{* *}\end{array}$ & $\begin{array}{c}0.00218 \\
(0.34) \\
\end{array}$ & $\begin{array}{c}-0.0039 \\
(-2.02)^{* *}\end{array}$ \\
\hline AVG & $\begin{array}{c}6.64 \\
(0.74)\end{array}$ & $\begin{array}{c}-3.99 \\
(-1.80)^{*}\end{array}$ & & \\
\hline $\mathrm{AVG}^{2}$ & $\begin{array}{r}-490.97 \\
(-1.17)\end{array}$ & $\begin{array}{c}-163.27 \\
(-2.54)^{* *}\end{array}$ & & \\
\hline$\overline{\mathrm{ABS}}$ & & & $\begin{array}{c}-3.67 \\
(-0.10) \\
\end{array}$ & $\begin{array}{l}-0.435 \\
(-0.08)\end{array}$ \\
\hline $\mathrm{ABS}^{2}$ & & & $\begin{array}{l}92.34 \\
(0.10)\end{array}$ & $\begin{array}{l}-5.137 \\
(-0.04)\end{array}$ \\
\hline $\begin{array}{l}\text { Lambda } \\
\text { (std. error) }\end{array}$ & $\begin{array}{c}-0.0340 \\
(0.0057)^{* * *}\end{array}$ & & $\begin{array}{c}-0.0341 \\
(0.0057)^{* * *}\end{array}$ & \\
\hline $\begin{array}{c}\text { Rho } \\
\text { (std. error) }\end{array}$ & $\begin{array}{c}-0.1329 \\
(0.0210)^{* * *}\end{array}$ & & $\begin{array}{c}-0.1331 \\
(0.0210)^{* * *}\end{array}$ & \\
\hline
\end{tabular}

Observations: 44,251 Firms: 5,876

Notes: See notes for Table 4.

To examine the magnitude of the effect of uncertainty on export behavior of firms, we compute its marginal effect. Table 7 presents the marginal effect calculated at the mean of uncertainty from Table 4. Concentrating on the effect of uncertainty on export share, the table shows that if exchange rate uncertainty (AVG) were to rise by 100 percent, export share would fall by about 4.4 percentage points, which is equivalent to a decrease of about 16.6 percent at the mean. ${ }^{2}$ It is similar to the results for knitting industry in Columbia in Das, Robert and Tybout (2007). The marginal effect of SD is bigger as its value is generally much smaller than that of AVG.

\footnotetext{
${ }^{2}$ This is computed using the mean of export share. From the estimates in table 4 the mean of export share is 0.2662 . So the change in percentage terms is $4.416 / 0.2662=16.6$.
} 
Table 7: Marginal Effects of the Heckman Selection Model for Table 4

\begin{tabular}{ccc}
\hline & $\begin{array}{c}\text { Export } \\
\text { Dummy }\end{array}$ & Export Share \\
\hline AVG & 0.579 & -4.416 \\
& $(1.73)$ & $(2.35)^{*}$ \\
SD & 6.269 & 18.20 \\
& $(5.946)$ & $(6.568)^{* * *}$ \\
\hline
\end{tabular}

*significant at $10 \%$; ** significant at $5 \%$; *** significant at $1 \%$

Then we examine the effects of uncertainty on different types of firms (foreign vs. domestic) in Table 8. The coefficients using ABS are always insignificant in sample selection models, as in the former regression. So the results using this measure cannot provide any evidence for this. Here we use AVG as uncertainty. Firstly we interact AVG with foreign owner dummy to evaluate the difference (domestic firms as the reference group). Column 1 reports the results by adding quadratic interaction terms in the regression. The linear and quadratic interaction terms are always insignificant in both export entry and export intensity decisions, which implies little difference between multinationals and indigenous firms. Then we split the sample into foreign owned firms and domestic firms, and rerun the regressions. The results are reported in Columns 2 and 3. As is the case with REERs, the coefficient of uncertainty in export share decision for foreign firms is insignificant, whereas that for domestic firm becomes strongly significant. Uncertainty is always insignificant in export participation decisions. The evidence shows that exchange rate uncertainty only negatively affects export intensity. Multinationals are less likely to be influenced by exchange rate uncertainty.

In order to investigate the industry heterogeneity, we split our sample by 2-digit 
industry and the coefficients of exchange rate uncertainty (using three different measures) are reported in Table 9. ${ }^{3}$ The results are different from the evidence using the pooled sample, as there are significant effects for some industries using $\mathrm{ABS}$ and $\mathrm{SD}$ as the measures. And we also observe some positive effects for some industries. However, different measures tell different stories for the same industry. The coefficients are unstable and hard to systematically analyze. However, the results strongly suggest industry heterogeneity and further investigating into industry characteristics is required to fully understand this issue.

Table 8: Heckman Selection Model with Uncertainty: Foreign vs. Domestic Firms

\begin{tabular}{|c|c|c|c|c|c|c|}
\hline & \multicolumn{2}{|c|}{$\begin{array}{l}\text { (1) AVG quadratic } \\
\text { interacting with foreign } \\
\text { dummy }\end{array}$} & \multicolumn{2}{|c|}{$\begin{array}{l}\text { (2) subsample: } \\
\text { foreign dummy=1 }\end{array}$} & \multicolumn{2}{|c|}{$\begin{array}{c}\text { (3) subsample: } \\
\text { foreign dummy=0 }\end{array}$} \\
\hline & $\begin{array}{l}\text { Export } \\
\text { Dummy }\end{array}$ & $\begin{array}{l}\text { Export } \\
\text { Share }\end{array}$ & $\begin{array}{l}\text { Export } \\
\text { Dummy }\end{array}$ & $\begin{array}{l}\text { Export } \\
\text { Share }\end{array}$ & $\begin{array}{l}\text { Export } \\
\text { Dummy }\end{array}$ & $\begin{array}{l}\text { Export } \\
\text { Share }\end{array}$ \\
\hline $\begin{array}{l}\text { Lag Industry } \\
\text { REER }\end{array}$ & $\begin{array}{l}0.002 \\
(0.31) \\
\end{array}$ & $\begin{array}{c}-0.004 \\
(-2.05)^{* *} \\
\end{array}$ & $\begin{array}{l}0.0018 \\
(0.22) \\
\end{array}$ & $\begin{array}{l}-0.003 \\
(-1.40) \\
\end{array}$ & $\begin{array}{l}0.0022 \\
(0.30) \\
\end{array}$ & $\begin{array}{l}-0.0046 \\
(-2.38)^{* *}\end{array}$ \\
\hline $\begin{array}{c}\text { Foreignown } \\
\text { dummy }\end{array}$ & $\begin{array}{c}0.14 \\
(4.19)^{* * *}\end{array}$ & $\begin{array}{c}0.06 \\
(7.03)^{* * *}\end{array}$ & & & & \\
\hline $\begin{array}{l}\text { Uncertainty } \\
\text { (AVG) }\end{array}$ & $\begin{array}{c}5.97 \\
(0.66)\end{array}$ & $\begin{array}{c}-4.11 \\
(-1.84)^{*}\end{array}$ & $\begin{array}{l}-7.48 \\
(-0.52)\end{array}$ & $\begin{array}{c}-2.26 \\
(-0.85)\end{array}$ & $\begin{array}{c}8.33 \\
(0.60)\end{array}$ & $\begin{array}{c}-7.33 \\
(-2.98)^{* * *}\end{array}$ \\
\hline $\begin{array}{c}\text { AVG* }^{*} \\
\text { foreignown } \\
\text { dummy }\end{array}$ & $\begin{array}{l}1.637 \\
(0.60)\end{array}$ & $\begin{array}{l}0.276 \\
(0.72)\end{array}$ & & & & \\
\hline $\mathrm{AVG}^{2}$ & $\begin{array}{l}-443.0 \\
(-1.09)\end{array}$ & $\begin{array}{c}-153.9 \\
(-2.45)^{* *}\end{array}$ & & & & \\
\hline $\begin{array}{c}\mathrm{AVG}^{2 *} \\
\text { foreignown } \\
\text { dummy }\end{array}$ & $\begin{array}{l}-132.0 \\
(-0.85)\end{array}$ & $\begin{array}{l}-21.9 \\
(-1.36)\end{array}$ & & & & \\
\hline $\begin{array}{c}\text { Lambda } \\
\text { (std. error) }\end{array}$ & \multicolumn{2}{|c|}{$\begin{array}{c}-0.0341 \\
(0.0056)^{* * *}\end{array}$} & \multicolumn{2}{|c|}{$\begin{array}{c}-0.0248 \\
(0.01057)^{* *}\end{array}$} & \multicolumn{2}{|c|}{$\begin{array}{c}-0.0399 \\
(0.0059)^{* * *}\end{array}$} \\
\hline $\begin{array}{c}\text { Rho } \\
\text { (std. error) }\end{array}$ & \multicolumn{2}{|c|}{$\begin{array}{c}-0.1332 \\
(0.021)^{* * *}\end{array}$} & \multicolumn{2}{|c|}{$\begin{array}{c}-0.092 \\
(0.0387)^{* * *}\end{array}$} & \multicolumn{2}{|c|}{$\begin{array}{c}-0.167 \\
(0.0246)^{* * *}\end{array}$} \\
\hline Observations & \multicolumn{2}{|c|}{44,245} & \multicolumn{2}{|c|}{20,571} & \multicolumn{2}{|c|}{23,674} \\
\hline
\end{tabular}

Notes: See notes for Table 4.

\footnotetext{
${ }^{3}$ We use the same way as in Chapter 2 to see if there are significant differences between 2-digit industries for the coefficients of uncertainty by generating 21 industry dummies and rearranging the dummies. The results generally show that the differences are significant. So we do not report the detailed results here.
} 
Table 9: Industry Heterogeneity in the Effects of Exchange Rate Uncertainty (Heckman selection models by 2-digit industry)

\begin{tabular}{|c|c|c|c|c|c|c|c|}
\hline \multirow[t]{2}{*}{$\begin{array}{l}\text { Industry } \\
\text { Code }\end{array}$} & \multicolumn{2}{|c|}{$\begin{array}{c}\text { (1) Uncertainty: } \\
\text { AVG }\end{array}$} & \multicolumn{2}{|c|}{$\begin{array}{c}\text { (2) Uncertainty: } \\
\text { ABS }\end{array}$} & \multicolumn{2}{|c|}{$\begin{array}{l}\text { (3) Uncertainty: } \\
\text { SD }\end{array}$} & \multirow{2}{*}{$\begin{array}{c}\text { Number } \\
\text { of } \\
\text { Observation }\end{array}$} \\
\hline & $\begin{array}{l}\text { Export } \\
\text { dummy }\end{array}$ & $\begin{array}{l}\text { Export } \\
\text { share }\end{array}$ & $\begin{array}{l}\text { Export } \\
\text { dummy }\end{array}$ & $\begin{array}{l}\text { Export } \\
\text { share }\end{array}$ & $\begin{array}{l}\text { Export } \\
\text { dummy }\end{array}$ & $\begin{array}{l}\text { Export } \\
\text { share }\end{array}$ & \\
\hline 15 & + & $-* * *$ & - & $+* * *$ & - & $+* * *$ & 1830 \\
\hline 17 & - & + & + & $+* * *$ & $t^{*}$ & $+* * *$ & 1271 \\
\hline 18 & $+* * *$ & $-* * *$ & + & + & + & $+^{*}$ & 641 \\
\hline 20 & $-* * *$ & + & - & $+* * *$ & - & + & 344 \\
\hline 22 & - & $-* * *$ & $-*$ & $-* * *$ & $+* * *$ & + & 6503 \\
\hline 24 & $+* *$ & + & $-* *$ & + & - & $+* * *$ & 2699 \\
\hline 26 & $-* * *$ & $-* * *$ & - & + & + & $+* * *$ & 1776 \\
\hline 27 & $-* * *$ & + & -* & + & + & $-* * *$ & 1486 \\
\hline 28 & + & $-* * *$ & + & + & + & + & 4808 \\
\hline 29 & + & - & $+* *$ & - & + & $+* * *$ & 5856 \\
\hline 31 & - & $-* * *$ & + & $+* *$ & + & $+* * *$ & 1008 \\
\hline 32 & $+* * *$ & $+* * *$ & $-* * *$ & $-* * *$ & $-* * *$ & $-* * *$ & 1528 \\
\hline 33 & $-* * *$ & $+* * *$ & $-* *$ & + & $-*$ & - & 720 \\
\hline 34 & + & + & $+* * *$ & - & + & + & 1736 \\
\hline 35 & $-* * *$ & $+*$ & $+* * *$ & + & $+* * *$ & - & 326 \\
\hline 36 & $+* * *$ & + & $-*$ & - & $-* * *$ & - & 5553 \\
\hline
\end{tabular}

*significant at $10 \% ;{ }^{* *}$ significant at $5 \%$; ${ }^{* *}$ significant at $1 \%$

We focus on the results using AVG as the measure. 5 out of 16 industries have a significant coefficient both for export participation decision and export share decision, which suggests the significant effect of uncertainty on export. Table 10 reports the summary of the effects across 2-digit industries with detailed industry names, which split the industries into 6 categories according to different effects in export entry and export intensity decisions. We list negative effect categories at the top, the insignificant effect category in the middle, and the positive effect and opposite effects (between export entry and export intensity) categories at the 
Table 10: Summary of Industry Heterogeneity (AVG as proxy for uncertainty)

\begin{tabular}{|c|c|}
\hline $\begin{array}{l}\text { Different } \\
\text { effect type }\end{array}$ & Industries \\
\hline $\begin{array}{l}\text { export entry } \\
\text { (negative), } \\
\text { export share } \\
\text { (insignificant } \\
\text { or negative) }\end{array}$ & $\begin{array}{l}20 \text { Manufacture of Wood And Products of Wood And Cork, Except } \\
\text { Furniture; Manufacture of Articles of Straw and Plaiting Materials } \\
26 \text { Manufacture of Other Non-metallic Mineral Products } \\
27 \text { Manufacture of Basic Metals }\end{array}$ \\
\hline $\begin{array}{l}\text { export entry } \\
\text { (insignificant) } \\
\text { export share } \\
\text { (negative) }\end{array}$ & $\begin{array}{l}15 \text { Manufacture of Food Products and Beverages } \\
22 \text { Publishing, Printing and Reproduction of Recorded Media } \\
28 \text { Manufacture of Fabricated Metal Products, Except Machinery and } \\
\text { Equipment } \\
31 \text { Manufacture of Electrical Machinery and Apparatus Not } \\
\text { Elsewhere Classified }\end{array}$ \\
\hline $\begin{array}{l}\text { Insignificant in } \\
\text { both decisions }\end{array}$ & $\begin{array}{l}17 \text { Manufacture of Textiles } \\
29 \text { Manufacture of Machinery and Equipment Not Elsewhere } \\
\text { Classified } \\
34 \text { Manufacture of Motor Vehicles, Trailers and Semi-trailers }\end{array}$ \\
\hline $\begin{array}{l}\text { export entry } \\
\text { (positive) } \\
\text { export share } \\
\text { (insignificant } \\
\text { or positive) }\end{array}$ & $\begin{array}{l}24 \text { Manufacture of Chemicals and Chemical Products } \\
32 \text { Manufacture of Radio, Television and Communication Equipment } \\
\text { and Apparatus } \\
36 \text { Manufacture of Furniture; Manufacturing Not Elsewhere } \\
\text { Classified }\end{array}$ \\
\hline $\begin{array}{l}\text { export entry } \\
\text { (positive) } \\
\text { export share } \\
\text { (negative) }\end{array}$ & 18 Manufacture of Wearing Apparel; Dressing and Dyeing of Fur \\
\hline $\begin{array}{l}\text { export entry } \\
\text { (negative), } \\
\text { export share } \\
\text { (positive) }\end{array}$ & $\begin{array}{l}33 \text { Manufacture of Medical, Precision and Optical Instruments, } \\
\text { Watches and Clocks } \\
35 \text { Manufacture of Other Transport Equipment }\end{array}$ \\
\hline
\end{tabular}

bottom. It is interesting to see that, in general, the industries with low levels of skills and technology incur negative effects of uncertainty on export decisions, whereas industries with high technology incur insignificant or positive effects. The results are consistent with the hypothesis of Darby et al. (1999) considering different characteristics across industries. However, there are three industries with 
contradicting effects for export entry and export share decision. There may be a combination of several roles that makes the results not so straightforward.

\section{Conclusion}

Our empirical results from AVG and ABS as measures of uncertainty provide an indirect way to test the hysteresis hypothesis. Exchange rate uncertainty was investigated in two ways: the magnitude of uncertainty and direction of uncertainty. Results show that the magnitude of the exchange rate uncertainty has little impact on export behavior, whereas the direction has a negative and significant impact on firms export intensity decision. And the impact of uncertainty with direction is nonlinear: increased uncertainty would induce bigger negative effects on export shares. The results provide some evidence for that increased exchange rate uncertainty would increase the inertia in firms export share decision.

We also find that multinationals are less negatively affected by exchange rate uncertainty than indigenous firms, which may confirm the advantage of multinationals with internationally extensive businesses. Industry heterogeneity in the effects is investigated in the end. We find that industry heterogeneity is important and there would be serious problems of aggregation using pooling and aggregate data. Future research should further investigate the characteristics of industries and their impacts on the role of uncertainty. Recent work of Bloom, Bond and von Reenen (2007) on the relationship of uncertainty and investment adopts an error correction model (ECM) and uses GMM to estimate in a dynamic framework, which may provide another interesting way to investigate the issue in a more complex context. 


\section{Chapter 6}

\section{Conclusions}

\section{Summary of Key Findings}

This thesis contributes to the micro econometric analysis of the effects of exchange rate movements on exports. We started by reviewing the theoretical work exploring the effects of exchange rate fluctuations on trade, as well as the empirical literature that focuses on the relationship between exchange rates and trade (exports) using macro and micro data. Theoretical models in this area basically argue that large enough temporary exchange rate volatility can have hysteresis effects on trade when market-entry costs are sunk. Early empirical studies focusing on aggregate relationships between exchange rate volatility and trade using macro data report little or no significant evidence for a negative effect, though it is widely believed exchange rate volatility has a negative impact on the level of trade. Recent empirical work adopting a gravity approach has found some evidence of a negative relationship. Most recently, a few papers using firm level micro data to examine the relationship between exchange rate movements and the export behaviour of firms finds ambiguous evidence.

In the preceding chapters, the thesis contributes to the empirical literature by addressing several issues that, to our knowledge, have not been investigated or fully investigated so far. Exchange rate movements can be regarded as having two aspects: changes of the level of exchange rates and secondly the effects of exchange rate uncertainty (called volatility in some cases). There is no general equilibrium theoretical model embedded in firm behaviour on the relationship 
between the level of exchange rates and exports. The negative effect between the two believed by many researchers and policy makers is partly fashioned by aggregate models and partly intuitive, as exchange rate appreciation may lead to price rising in foreign markets and thus may induce reduction in demand. All existing theoretical models in this area focus on the relationship between exchange rate uncertainty and trade. Empirical work on these two aspects is inconclusive.

In Chapter 2, we examined the effects of exchange rate level changes on firm decisions on export market entry, exit and export intensity, using data on a large sample of UK manufacturing firms. The empirical analysis breaks down export adjustments between changes in export share by existing exporters and changes due to entry to and exit from export markets. We find that level changes have little effect on firm export participation and exit decisions. However, they do have a significant negative impact on export shares after entry. The responsiveness of the export share to exchange rate changes is not quantitatively small: one index point depreciation in the REER index will increase export share by about 1.28 percent. The data also enable us to investigate the effects of changes of exchange rate level on the export behaviour of multinationals, and we find their export behaviour is less likely to be negatively affected by exchange rate changes than that of indigenous firms. This, among other things, offers a new way of examining the export behaviour of multinationals in response to exchange rate variability. According to the standard textbooks of international business, MNEs can internalise currency risk in many ways, and may be less affected by exchange rate movements. However, to our knowledge, there is no evidence on this issue. We 
also find that industry heterogeneity is important in the effects of exchange rate level changes on exports.

We then tried to explain the industry heterogeneity in the effects of exchange rate level changes. If industry heterogeneity is important, the evidence from aggregate data may have a serious problem of aggregation, which leads to questionable results. We explained the industry heterogeneity by testing two hypotheses in the following two chapters.

Chapter 3 is about the cost impact of exchange rate changes. Real exchange rate appreciation obviously makes the export price more expensive, but it also makes imported inputs cheaper, which may lead to cost reduction. So the degree of exposure to external shocks for each industry may have some role on the effects of exchange rate movements on export. Controlling for external orientation (the level of imported intermediate inputs) may partly explain industry heterogeneity. We rely on two families of indicators for each 2-digit manufacturing industry, intended to proxy for the inward orientation (Family 1,2 measures), and the net external orientation which combining inward orientation with outward orientation (Family 2 , 3 measures). We find some evidence of the role of external orientation: industries with a low degree of external orientation are less likely to be affected negatively by exchange rate movements.

Chapter 4 examined the role of market structure, specifically the role of pricing to market behaviour. We borrowed the idea of PTM from the literature on exchange rate pass-through. The main idea is that under imperfect competition when home 
and foreign varieties are close substitutes, price setters prefer to keep their prices closely in line with those of local industry competitors if they are able to do so. Thus the export prices of firms are detached from domestic prices with segmented markets. PTM ensures price stability in terms of export destination local currency. We expect that exports in sectors with a high degree of PTM are less negatively affected by exchange rate movements in terms of local currencies. We chose intraindustry trade type indices as the measures of the determinant of PTM and market power to test whether PTM plays a role in the relationship between exchange rate movements and exports. The results support the role of PTM: industries with high level of intra-industry trade are less likely impacted by exchange rate changes.

Chapters 3 and 4 contribute to the empirical literature by providing the first attempt, to our knowledge, to explain industry heterogeneity in the effects of exchange rate variability on exports. Controlling for external orientation and market structure is a new approach to explaining this issue. Accordingly, our results offer new insights with potential implications for policy makers: different policies should be employed for different industries, as the effects of exchange rate fluctuations on exports vary with different degrees of exposure to external shocks and different market structures for different industries.

Chapter 5 turns to investigate the impact of exchange rate uncertainty on firms' export decisions using the same data. Theoretical models argue there are hysteresis effects of exchange rate uncertainty on trade price and quantities, and that the size of the gap between the exchange rates that trigger entry and exit is not constant but increasing with uncertainty. We tested this hypothesis of hysteresis. There are 
difficulties in the empirical test: first, theory does not provide a direct testable proposition to examine hysteresis effects, as it is difficult to compute the thresholds of entry decision and examine the relationship between the thresholds and uncertainty; second, existing papers just focus on investigating simple correlations between trade and exchange rate uncertainty, which is highly questionable (model misspecification); third, there is difficulty in observing the effects on export entry because of the rarity of entry behaviour. So we may not observe the effect of exchange rate uncertainty on export entry, but might observe effects on export intensity. In this chapter, we used new measures of exchange rate uncertainty to test the hypothesis. Traditional measures are variance or standard deviation, all bounded and non-negative. Our measures are the average of monthly differences and average of absolute monthly differences between the previous period's forward rate and the current spot rate. The former can be either positive or negative to proxy for the direction of asymmetric uncertainty; the later is non-negative to proxy for the magnitude of uncertainty. Thus, exchange rate uncertainty is investigated in two ways: the size of uncertainty and its direction. Our results show that size has little impact on export behaviour, whereas direction has a significant impact. If the persistent band increases with the magnitude of uncertainty according to the theory, no significant effects of uncertainty on trade would be found. If the directions of uncertainty are asymmetric, we may observe a significant negative effect using the proxy for direction of uncertainty in export intensity decision. Our results confirm that and thus provide significant evidence for the hypothesis of hysteresis effects. We also find that the effect of uncertainty direction is nonlinear: increased uncertainty would induce a greater degree of negative impact on export intensity. In the final part of the chapter, we examine whether different ownership matters in 
the relationship and whether there is industry heterogeneity, again we find that multinationals are less likely to be influenced by exchange rate uncertainty and industry heterogeneity is important.

\section{Limitations and Suggestions for Future Research}

Our empirical analysis is based on a reduced-form econometric model which is a function of observable variables, following some of the existing micro empirical papers. Thus, the derivation of the econometric specification is pragmatic. One challenge for future work is to build a heterogeneous firm theoretical model to derive a theory consistent econometric specification.

In this thesis, we examined several factors influence the role of exchange rate changes on exports by exploring different sets of interaction effects one by one rather than putting all of the interaction terms in a regression. There are two main reasons for that. One is that we used several measures to proxy for each factor to examine its role, and no one single measure can fully proxy for the factor perfectly. So including just one of the measures may not be a sensible way. But including several measures may bring in other econometric problem. The other reason is that two different factors we examined are believed to be orthogonal to each other. So whether or not to include one of them would not influence the role of the other. If we can decide which measures can fully represent the factors, we can include these measures in our regression simultaneously to see their impact on firms.

The econometric methodology we use is a Heckman sample selection type model. We have discussed limitations of the model in Chapter 2. If relevant software 
programs are available, we can use them to deal with the econometric problems and to check robustness of our results. The distribution of the error terms from the selection equation and outcome equation is assumed to be bivariate normal with correlation $\rho$. One of the limitations of the selection model is that it is very sensitive to the assumption of bivariate normality. Although we have discussed in Chapter 2 that violation of the normality assumption may not cause serous bias in the parameter estimates (van der Klaauw and Koning, 2003), a test for the normality assumption in the sample selection model (such as van der Klaauw and Koning, 2003) can be done. If normality is rejected, consistent parameter estimation such as a semi-nonparametric method proposed by Gallant and Nychka (1987) can be used. As we use firm level panel data in the thesis, the most typical concern in empirical work using panel data has been the presence of unobserved heterogeneity. A procedure proposed by Vella and Verbeek (1999) can be used to deal with the problem. Moreover, if the dynamic selection model suggested by most recent papers such as Gayle and Viauroux (2007) and Raymond et al (2007) can be used, we can also include some dynamics in the model. The problem of endogeneity may be dealt with by using other instrument variables such as Lachenmaier and Woessmann (2006).

In Chapter 2, due to data limitation, the data for firms' ownership is their status in 2004 and we have to assume that the ownership of a firm in 2004 is the same throughout the time period. In addition, some UK domestic firms are multinationals. But we have to regard all of them as non-multinationals. More comprehensive firm level ownership data for each year would enrich the analysis. As this is the first paper analysing the different export behaviour of multinationals from those of 
domestic firms under exchange rate fluctuation, it would be interesting to go further in this aspect and look at specific characteristics of multinationals and domestic firms to explain observed differences. However, further investigation on this would require more informative firm level dataset.

Chapter 3 uses various measures of external orientation to examine whether the degree of imported intermediate inputs plays a role in the effects of exchange rate variability on exports. However, due to the classification system being on inputoutput table, we cannot calculate the measures for more disaggregate industry level but rather rely on 2-digit level data. Also the calculation of the measures is limited: only partly capturing the degree of external orientation. A better way to proxy for that, such as disentangling the tradable and non-tradable of the intermediate inputs, taking the difference between producer prices and purchaser's prices into consideration, would be helpful to further explore our results.

Chapter 4 explains industry heterogeneity of the effects from an interesting and novel angle. Market structure usually refers to a country's domestic market. Intraindustry trade indices sidestep the difficulty of measuring the market structure of exporting markets and still carry some related information about market structure, which is novel. A formal theoretical model to show the role of PTM on the effects of exchange rate movements on exports will be quite. Other new ways to measure the degree of PTM will provide alternative measures to check robustness of our results for the role of PTM on effects of exchange rate movements.

Chapter 5 explores the relationship between exchange rate uncertainty and exports, which is relevant to the hot topic of uncertainty and investment since 1990's. 
Recent work of Bloom, Bond and van Reenen (2007) on the relationship between uncertainty and investment adopts an error correction model (ECM) and uses GMM to estimate the dynamic framework, which may provide another interesting way to investigate the issue in a more complex context. As we find industry heterogeneity in the effects of exchange rate uncertainty on exports, a further exploration on what factors make the differences across industries can be an attractive direction for future work. ${ }^{1}$ However, this is subject to data availability If we had data on export destinations, or greater balance sheet information, or detailed survey data, we could do more insightful analysis on this issue, such as more specific exchange rates could be computed, the market structure of specific export destinations could be evaluated, some financial instruments could be used, and whether export destination is a factor to influence the effects of exchange rate fluctuations on exports. More detailed firm level data would enrich the study and help us to fully understand this issue.

This thesis focuses on the case of the UK. Would similar relationships hold for other developed and developing countries? Since the 1980's after the Plaza Accord, the Japanese Yen has experienced dramatic appreciation, but exporting firms in Japan seem to be negatively affected to a much lesser extent. What makes Japanese exporting firms swallow the consequence of the appreciation with a less negative impact? Can this happen to developing countries? Evidence from big developing countries such as China could generate new insights on the issue. Empirical analysis for developing countries especially transition economies is attractive as the

\footnotetext{
'We have tried several measures of market structure such as price-cost margins, R\&D intensity to see if there is any role of market power on the effects, but we failed to find any significant elements to explain the industry heterogeneity.
} 
currency policy in these countries is becoming more flexible, which may lead to increasing exchange rate volatility. Under such circumstances, will the economy in these countries be more vulnerable than developed countries? What can the results provide by way of policy implications? These questions are very interesting and worthwhile investigating. However, data availability and quality may restrict further research in this aspect.

\section{Conclusions}

The aim of the thesis is to examine effects of exchange rate variability on firms' export decisions, using data for a sample of UK manufacturing firms. We separately investigate the relationship between the changes of level of exchange rate and exports and that between exchange rate uncertainty and exports. Our results show that firm export participation and exit decisions are not significantly related to changes in the level of exchange rates. The exchange rate has a significant and negative impact on the export intensity after entry however. The responsiveness of export share on the degree of exchange rate changes is not quantitatively small. Since industry heterogeneity is important in the effects of exchange rate level changes on exports, we further explore possible explanations. In particular, we test whether external orientation and market structure play a role in the effects. Our results provide significant evidence for a role of external orientation and market structure. We also find significant evidence for the hysteresis effect of exchange rate uncertainty on exports, using newl measures of uncertainty. In both cases, we investigate the behaviour of multinationals and the results show that multinationals are less likely to be negatively affected by both 
changes of level of exchange rate and exchange rate uncertainty than indigenous firms. 


\section{References}

Alvarez, Roberto and Görg, Holger(2005), "Multinationals and Plant Exit:

Evidence from Chile', GEP Research Paper 05/16, Leverhulme Centre for

Research on Globalisation and Economic Policy, University of Nottingham. Anderson, James (1979), “A Theoretical Foundation for the Gravity Equation," American Economic Review, Vol. 69: 106-116.

Asseery, A. and D. A. Peel (1991), "The Effects of Exchange Rate Volatility on

Exports - Some New Estimates", Economics Letters, Vol. 37: 173-77.

Athukorala, P. and Menon, J. (1994), "Pricing to Market Behaviour and Exchange

Rate Pass-through in Japanese Exports”, Economic Journal, Vol. 104, No.

423: $271-281$.

Atkeson, Andrew and Burstein, Ariel (2008), "Pricing-to-Market, Trade Costs, and International Relative Prices", working paper.

Baldwin, Richard (1988), "Hysteresis and the Beachhead Effect," American

Economic Review, Vol. 78: 773-85.

Baldwin, Richard and Krugman, Paul (1989), "Persistent Trade Effects of Large

Exchange Rate Shocks," Quarterly Journal of Economics, Vol. 104:635-54.

Baldwin, Richard and Ottaviano, G. (2001), "Multiproduct Multinationals and

Reciprocal FDI Dumping," Journal of International Economics, Vol. 54,

No. 2: 429-448.

Bayoumia, Tamim and Eichengreen, Barry (1998), "Exchange Rate Volatility and

Intervention: Implications of the Theory of Optimum Currency Areas", Journal of International Economics, Vol. 45, No. 2: 191-209.

Bélanger, Denis, Sylvia Gutiérrez and Jacques Raynauld (1988), "Exchange Rate Variability and Trade Flows: Sectoral Estimates for the US-Canada Case." 
Cahiers de Recherche IEA-89-01. Institut d'économie appliquée, École des Hautes Études Commerciales.

Bélanger, Denis, Sylvia Gutiérrez, Daniel Racette and Jacques Raynauld (1992), "The Impact of Exchange Rate Variability on Trade Flows: Further Results on Sectoral U.S. Imports from Canada", North American Journal of Economics and Finance, Vol. 3: 888-892.

Bernard, A. and Jensen, J.B. (1995), "Exporters, jobs and wages in US manufacturing: 1976-1987”, Brookings Papers on Economic Activity, Microeconomics: $67-119$.

Bernard, A. and J. Jensen (1999), "Exceptional Exporters Performance: Cause, Effect or Both?" Journal of International Economics, Vol. 47: 1-25.

Bernard, A. and Jensen, J. (2004a), "Why Some Firms Export", Review of Economics and Statistics, Vol. 86: 561-569.

Bernard, A. and Jensen, J. (2004b), "Entry, Expansion and Intensity in the U.S. Export Boom, 1987-1992" Review of International Economics, Vol. 12: $662-675$.

Bernard, Andrew B., Jensen, J. Bradford and Schott, Peter K. (2003), "Falling Trade Costs, Heterogeneous Firms, and Industry Dynamics", NBER Working Paper 9639.

Bernard, A. and Wagner, J. (2001), "Export Entry and Exit by German Firms", Weltwirtschaftliches Archiv, Vol. 137: 134-157.

Blalock, G. and Roy, S. (2007), "A Firm Level Examination of the Exports Puzzle: Why East Asian Exports Didn't Increase after 1997-1998 Financial Crisis?”, The World Economy, Vol. 30: 39-59. 
Bloom, N., Bond, S. and Van Reenen, J. (2007), "Uncertainty and Investment Dynamics", Review of Economic Studies, Vol. 74, No. 2: 391-415.

Bond, S. R. and Cummins, J. G. (2004), "Uncertainty and Investment: an Empirical Investigation Using Data on Analysts' Profits Forecasts", Finance and Economics Discussion Series 2004-20.

Brambor, T., Clark, W. R. and Golder, M. (2006), "Understanding Interaction Models: Improving Empirical Analyses", Political Analysis, Vol. 14, No. 1: 63-82.

Braumoeller, Bear (2004), "Hypothesis Testing and Multiplicative Interaction Terms", International Organization, Vol. 58:807-820.

Broda, C. and Weinstein, D.E. (2006), "Globalization and the Gain from Variety", Quarterly Journal of Economics, Vol.121, No. 2: 541-585,

Bugamelli, M. and Infante, L. (2003), "Sunk costs to exports", Bank of Italy Discussion Paper No. 469.

Bugamelli, M. and Tedeschi, R. (2008), "Pricing-to-Market and Market Structure", Oxford Bulletin of Economics and Statistics, Vol. 70:155-180.

Caballero, R. J. (1991), "On the Sign of the Investment-Uncertainty Relationship", The American Economic Review, Vol. 81, No. 1: 279-288.

Caballero and Pindyck (1996), "Uncertainty, Investment and Industry Evolution", International Economic Review, Vol.37: 641-662.

Campa, J. M. (1993), "Entry by Foreign Firms in the United States under Exchange Rate Uncertainty", Review of Economics and Statistics, Vol. 75, No. 4: 614622.

Campa, J. M. (2004), "Exchange Rates and Trade: How Important is Hysteresis in Trade?", European Economic Review, Vol. 48: 527-548. 
Campa, J. M. and Goldberg, L. (1995), "Investment in Manufacturing, Exchange

Rates and External Exposure”, Journal of International Economics, Vol. 38: 297-320.

Campa, J. M. and Goldberg, L. (1997), “The Evolving External Orientation of Manufacturing Industreis: Evidence from Four Countries", NBER Working Paper No. 5919.

Campa, J. M. and Goldberg, L. (1999), "Investment, Pass-Through, and Exchange Rates: A Cross-Country Comparison", International Economic Review, Vol. 40, No. 2: 287-314.

Campa, J. M. and Goldberg, L. (2001), "Employment versus Wage Adjustment and the U.S. Dollar", The Review of Economics and Statistics, Vol. 83, No. 3: 477-489.

Campa, J. M. and Goldberg, L. (2006), "Distribution Margins, Imported Inputs, and the Sensitivity of the CPI to Exchange Rates", NBER Working Paper No. 12121 .

Carruth, A., A. Dickerson and A. Henley (2000a), "What Do We Know about Investment under Uncertainty?", Journal of Economic Surveys, Vol. 14, No. 2: 119-153.

Carruth, A., A. Dickerson, and A. Henley (2000b), "Econometric Modelling of UK Aggregate Investment: The Role of Profits and Uncertainty", Manchester School, Vol. 68, No. 3: 276-300.

Cheiher, Andrew and Irish, Margaret (1987), "Residual Analysis in the Grouped Data and Censored NonnaJ Linear Model", Journal of Econometrics, Vol. 34: 33-62.

Cho, G., Sheldon, I. and McCorriston, S. (2002), "Exchange Rate Uncertainty and 
Agricultural Trade", American Journal of Agricultural Economics, Vol. 84, No. 4: $931-942$.

Choudhri, Ehsan U., Faruqee, Hamid and Hakura, Dalia S. (2005), "Explaining the Exchange Rate Pass-through in Different Prices", Journal of International Economics, Vol. 65: 349-374.

Clark, Peter B. (1973), "Uncertainty, Exchange Risk, and the Level of International Trade", Western Economic Journal, Vol. 11: 302-13.

Clark et al (2004), "A New Look at Exchange Rate Volatility and Trade Flows", IMF Occasional Paper 235

Clark, Peter B. and Faruqee, Hamid (1997), “Exchange Rate Volatility, Pricing to Market and Trade Smoothing", IMF Working Papers 97/126, International Monetary Fund.

Clerides, S., Lach, S. and Tybout, J. (1998), "Is Learning by Exporting Important? Micro-dynamic Evidence from Columbia, Mexico and Morocco", Quarterly Journal of Economics, Vol. 113, 903-948.

Cronbach, L. (1987), "Statistical Tests for Moderator Variables: Flaws in Analysis Recently Proposed", Psychological Bulletin, Vol. 102: 414-417.

Corsetti, G. and Dedola, L. (2002), “Macroeconomics of International Price Discrimination", unpublished paper.

Côté, A. (1994), "Exchange Rate Volatility and Trade", Bank of Canada Working Paper 94-5.

Cushman, David O. (1983), "The Effects of Real Exchange Rate Risk on International Trade", Journal of International Economics, Vol. 15: 43-63.

Darby, J., A. Hughes-Hallett, J. Ireland and L. Piscitelli (1999), "The Impact of Exchange Rate Uncertainty on the Level of Investment", The Economic 
Journal, Vol. 109: C55-C67.

Das, S., Roberts, M.J. and Tybout, J. (2007), “Market Entry Costs, Producer

Heterogeneity, and Export Dynamics", Econometrica, Vol. 75, No. 3: 837873

Dell'Ariccia, G. (1999), “Exchange Rate Fluctuations and Trade Flows: Evidence from the European Union", IMF Staff Papers 46, No. 3: 315-334.

Desai, M. A., Foley, F. C. and Forbes, K. J. (2004), "Financial Constraints and Growth: Multinational and Local Firm Responses to Currency Crises", NBER Working Paper No. 10545.

Dixit, Avinash (1989a), "Hysteresis, Import Penetration, and Exchange Rate PassThrough", The Quarterly Journal of Economics, Vol. 104, No. 2: 205-228. Dixit, Avinash (1989b), "Entry and Exit Decisions under Uncertainty", The Journal of Political Economy, Vol. 97, No. 3: 620-38.

Dixit, A. and R. S. Pindyck (1994), Investment under Uncertainty, Princeton, NJ: Princeton University Press.

Dornbusch, Rudiger (1987), "Exchange Rates and Prices", American Economic Review, Vol. 77: 93-106.

Driver, C. and D. Moreton (1991), "The Influence of Uncertainty on UK Manufacturing Investment”, Economic Journal, Vol. 101: 1452-1459. Dunlap, W. P. and Kemery, E. (1987), "Failure to Detect Moderating Effects: Is Multicollinearity the Problem?", Psychological Bulletin, Vol. 102: 418-420.

Dustmann, C. and M. E. Rochina-Barrachina (2000), "Selection Correction in Panel Data Models: An Application to Labour Supply and Wages", IZA Discussion Paper No. 162.

Faruqee, Hamid (1995), "Pricing to Market and the Real Exchange Rate", IMF 
Working Papers 95/12, International Monetary Fund.

Feenstra, Robert C., (1989), "Symmetric Pass-Through of Tariffs and Exchange Rates under Imperfect Competition: an Empirical Test", Journal of International Economics, Vol. 27: 25-45.

Feenstra, R. C. (1994), "New Product Varieties and the Measurement of International Prices", American Economic Review, Vol. 84: 157-177.

Feenstra, Robert C. and Kendall, Jon D. (1997), "Pass-through of Exchange Rates and Purchasing Power Parity", Journal of International Economics, Vol. 43: 237-261.

Ferderer, J. P. (1993), "The Impact of Uncertainty on Aggregate Investment Spending: An Empirical Analysis", Journal of Money, Credit, and Banking, Vol. 25, No. 1: 30-48.

Forbes, K., (2002), “How Do Large Depreciations Affect Firm Performance”, NBER Working Paper No. 9095.

Fontagne, L., Freudenberg, M. and Gaulier, G. (2006), "A Systematic Decomposition of World Trade into Horizontal and Vertical IIT", Review of World Economics, Vol. 142, No.3: 459-475.

Frankel, Jeffrey A., and S. J. Wei (1993), "Trade Blocs and Currency Blocks", NBER Working Paper No. 4335.

Froot, K., and Klemperer, P., (1989), "Exchange Rate Pass-through When Market Share Matters", American Economic Review, Vol. 79: 637-654.

Gallant. A. Ronald and Nychka, Douglas (1987), "Semi-Nonparametric Maximum Likelihood Estimation", Econometrica, Vol. 55, No. 2:363-90.

Gayle, G-L. and C. Viauroux (2007), "Root-N Consistent Semiparametric Estimators of a Dynamic Panel-Sample-Selection Model", Journal of 
Econometrics, Vol.141: 179-212.

Girma, S., Greenaway, D. and Kneller, R. (2003), "Export Market Exit and Performance Dynamics: A Causality Analysis of Matched Firms", Economic Letters, 80:181-187.

Girma, S., Greenaway, D. and Kneller, R. (2004), "Does Exporting Lead to Better Performance? A Microeconometric Analysis of Matched Firms", Review of International Economics 12(5): 855-866.

Goldberg, L. (1993), "Exchange Rates and Investment in United States Industry", Review of Economics and Statistics, Vol. 75: 575-588.

Goldberg, Pinelopi K. and Hellerstein, Rebecca (2007), "A Framework for Identifying the Sources of Local-Currency Price Stability with an Empirical Application", NBER Working Paper No.13183.

Goldberg, Pinelopi K. and Knetter, Micheal M (1997), "Goods Prices and

Exchange Rates: What Have We Learned", Journal of Economic Literature, Vol. 35: 1243-1272.

Goldberg, Pinelopi K. and Knetter, Micheal M (1999), "Measuring the Intensity of Competition in Export Markets", Journal of International Economics, Vol. 47: $27-60$.

Greene (2003), Econometric Analysis, fifth edition, Prentice-Hall.

Greenaway, D., Guariglia, A. and Kneller, R. (2007), "Financial Factors and Exporting Decisions", Journal of International Economics, forthcoming. Greenaway, D., Hine, R. and Milner, C. (1995), "Vertical and Horizontal Intraindustry Trade: a Cross Industry Analysis for the United Kingdom", The Economic Journal, Vol. 158: 1505-1518.

Greenaway, D., and Kneller, R. (2003), "Exporting, Productivity and 
Agglomeration: a Matched Difference in Difference Analysis of Matched Firms", GEP Research Paper 03/45, Leverhulme Centre for Research on Globalisation and Economic Policy, University of Nottingham

Greenaway, D. and Kneller R. (2004), "Firm Heterogeneity, Exporting and Foreign Direct Investment", The Economic Journal, Vol. 117: F134-F161.

Greenaway, D. and Kneller R. (2007), "Exporting and Productivity in the UK", Oxford Review of Economic Policy, Vol.20: 358-371.

Greenaway and Milner (1993), Trade and Industrial Policy in Developing

Countries, Macmillan, London; Michigan University Press, New York. Greenaway, D., Milner, C. and Elliott, R.J.R. (1999), “UK Intra-Industry Trade with the EU North and South", Oxford Bulletin of Economics and Statistics, Vol. 61, No.3: 365-384.

Grubel, Herbert G. and Lloyd, Peter J. (1975) Intra-Industry Trade: The Theory and Measurement of International Trade in Differentiated Products, New York: Wiley.

Gourieroux, C., Monfon, A., Renault, E.and Trognon, A. (1987), "Generalised Residuals", Journal of Econometrics, Vol. 34: 5-32.

Hadi, A. S. (1992), "Identifying Multiple Outliers in Multivariate Data", Journal of the Royal Statistical Society, Series B (Methodological), Vol. 54: 761-771.

Hadi, A. S. (1994), "A Modification of a Method for the Detection of Outliers in Multivariate Samples", Journal of the Royal Statistical Society, Series B (Methodological), Vol. 56: 393-396.

Heckman, James J. (1979), "Sample Selection Bias as a Specification Error", Econometrica, Vol. 47:153-61. 
Henley, A., A. Carruth and A. Dickerson (2003), "Industry-Wide versus FirmSpecific Uncertainty and Investment: British Company Panel Data Evidence", Economics Letters, Vol. 78, No. 1: 87-92.

Hill, CW (2005), International Business: Competing in the Global Marketplace, 5th ed, McGraw-Hill.

Huizinga, J. (1993), "Inflation Uncertainty, Relative Price Uncertainty, and Investment in U.S. Manufacturing”, Journal of Money, Credit, and Banking, Vol. 25, No.3: 521-557.

Hummels, David (1999), "Toward a Geography of Trade Costs", GTAP Working Papers 1162, Center for Global Trade Analysis, Department of Agricultural Economics, Purdue University.

IMF (1984), "The Exchange Rate System: Lessons of the Past and Options for the Future", IMF Occasional Paper No. 30 (Washington: IMF).

Jaccard, J. and Turrisi, R. (2003), Interaction Effects in Multiple Regression $\left(2^{\text {nd }}\right.$ ed.), Sage, University Paper Series on Quantitative Applications in the Social Sciences, series no. 07-072. Thousand Oaks, CA: Sage.

Jaccard, J., Wan, C. K. and Turrisi, R. (1990), "The Detection and Interpretation of Interaction Effects between Continuous Variables in Multiple Regression", Multivariate Behaviour Research, Vol. 25, No. 4: 467-478.

Karpaty, P. and Kneller, R. (2005), "Demonstration or Congestion? Export Spillovers in Sweden", GEP Research Paper 05/44, Leverhulme Centre for Research on Globalisation and Economic Policy, University of Nottingham. Kasa, Kenneth A. (1992), "Adjustment Costs and Pricing-to-Market: Theory and Evidence", Journal of International Economics, Vol. 32: 1-30.

Knetter, MICHAEL M. (1993), "International Comparisons of Pricing-to-Market 
Behavior", American Economic Review, Vol. 83, No. 3: 473-486.

Knetter, MICHAEL M. (1995), "Pricing to Market in Response to Unobservable and Observable Shocks", International Economic Journal, Vol. 9, No. 2: $1-25$.

Kneller, Richard and Pisu, Mauro (2005), "Industrial Linkages and Export Spillovers from FDI", GEP Research Paper 05/20, Leverhulme Centre for Research on Globalisation and Economic Policy, University of Nottingham. Krugman, Paul R. (1987), "Pricing to Market When the Exchange Rate Changes", in S. W. Arndt and J. D. Richardson, eds., Real-Financial Linkages Among Open Economies, Cambridge, MA: MIT Press, 1987, 49-70.

Krugman, Paul (1989), Exchange Rate Instability (MIT Press, Cambridge, MA: MIT Press

Kyriazidou, Ekaterini (1997), "Estimation of a Panel Data Sample Selection Model", Econometrica, Vol. 65, No. 6: 1335-1364.

Kyriazidou, Ekaterini (2001), "Estimation of Dynamic Panel Data Sample Selection Models", Review of Economic Studies, Vol. 68: 543-572.

Lachenmaier, S. and Woessmann, L. (2006), "Does Innovation Cause Exports?

Evidence from Exogenous Innovation Impulses and Obstacles Using German Micro Data", Oxford Economic Papers, Vol. 58, No. 2: 317-350.

Lastrapes, William D. and Koray, Faik (1990), "Exchange Rate Volatility and U.S.

Multilateral Trade Flows", Journal of Macroeconomics, Vol. 12: 341-62.

Leahy, J. and Whited, T. M. (1996), "The Effects of Uncertainty on Investment:

Some Stylized Facts", Journal of Money, Credit and Banking, Vol. 28, No.1: 64-83.

Lee, Hangyong (2005), "The Impact of Uncertainty on Investment: Empirical 
Evidence from Manufacturing Firms in Korea", Korea and the World Economy Conference paper, University of Washington, Seattle, U.S.A, December 10-11.

Lipsey, Robert E. (2001), "Foreign Direct Investors in Three Financial Crises", NBER Working Paper No. 8084.

Maddala, G.S., (1983), Limited-Dependent and Qualitative Variables in Econometrics, Cambridge University Press.

Makin, John H. (1978), "Portfolio Theory and the Problem of Foreign Exchange Risk", Journal of Finance, Vol. 33: 517-34.

Marston, R. (1990), "Pricing to Market in Japanese Manufacturing", Journal of International Economics, Vol. 29: 217-236.

McKenzie, Michael D. (1999), "The Impact of Exchange Rate Volatility on International Trade Flows", Journal of Economic Surveys, Vol. 13, No. 1: 71-106.

Nijman, Theo. and Verbeek, Mamo (1992), "Nonresponse in Panel Data: The Impact on Estimates of the Life Cycle Consumption Function", Journal of Applied Econometrics, Vol. 7, No. 3: 243-57.

Pagan, A. and Ullah, A. (1988), "The Econometric Analysis of Models with Risk Terms", Journal of Applied Econometrics, Vol. 3: 87-105.

Pavitt, K. (1984), "Sectoral Pattterns of Technical Change: towards a Taxonomy and a Theory", Research Policy, Vol. 13: 343-373.

Rangan, S. and Lawrence, R. Z. (1993), "The Response of U.S Firms to Exchange Rate Fluctuations: Piercing the Corporate Veil", Brookings Papers on Economic Activity, Vol.2: 341-369.

Raymond, W., Mohnen, P., Palm, F. and van der Loeff, S. S. (2007), "The 
Behavior of the Maximum Likelihood Estimator of Dynamic Panel Data Sample Selection Models", working paper

Roberts, Mark and Tybout, James (1997), "The Decision to Export in Colombia", American Economic Review, Vol. 87: 545-564

Romer, Paul (1994), "New Goods, Old Theory, and the Welfare Costs of Trade

Restrictions", Journal of Development Economics, Vol. 43, No.1: 5-38.

Rose, Andrew K (2000), “One Money, One Market: The Effect of Common

Currencies on Trade", Economic Policy, Vol. 15, No. 30: 7-45.

Price, S. (1996), “Aggregate Uncertainty, Investment and Asymmetric Adjustment in the UK Manufacturing Sector", Applied Economics, Vol. 28: 1369-1379.

Rugman, AM and S Collinson (2006), International Business, 4th ed, FT Prentice Hall.

Semykina, A. and J. M. Wooldridge (2005), "Estimating Panel Data Models in the Presence of Endogeneity and Selection: Theory and Application", unpublished working paper, Michigan State University.

Tenreyro, Silvana (2003), "On the Trade Impact of Nominal Exchange Rate Volatility", Unpublished, Federal Reserve Bank of Boston.

Thursby J.G., and M.C. Thursby (1987), "Bilateral Trade Flows, the Linder Hypothesis, and Exchange Risk", The Review of Economics and Statistics, Vol. 69: 488-495.

van der Klaauw, Bas and R. H. Koning (2003), "Testing the Normality Assumption in the Sample Selection Model with an Application to Travel Demand", Journal of Business \& Economic Statistics, Vol. 21, No. 1: 31-42.

Vella, Francis (1998), "Estimating Models with Sample Selection Bias: A Survey", The Journal of Human Resources, Vol. 33, No. 1: 127-169 
Vella, Francis, and Verbeek, Mamo (1999), “Two-Step Estimation of Panel Data Models with Censored Endogenous Variables and Selection Bias", Journal of Econometrics, Vol. 90: 239-263.

Wei, Shang-Jin (1999), "Currency Hedging and Goods Trade", European Economic Review, Vol. 43: 1371-1394.

Wooldridge, J. (2006), Introductory Econometrics: A Modern Approach, 3rd Edition, South-Western.

Yang, Jiawen (1998), "Pricing-to-market in U.S. Imports and Exports: a Time Series and Cross-sessional Study", Quarterly Review of Economics and Finance, Vol. 38, No.4: 843-861. 